\title{
NONTOPOLOGICAL SOLITONS
}

\author{
T.D. LEE
}

Department of Physics, Columbia University, New York, NY 10027, USA

and

Y. PANG

Brookhaven National Laboratory, Upton, NY 11973, USA

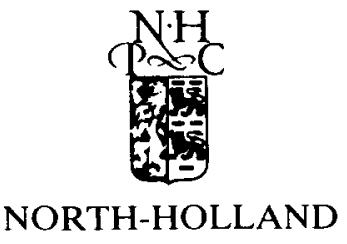




\section{Nontopological solitons*}

T.D. Lee

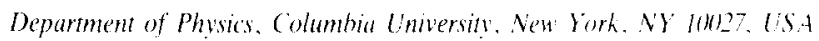

and

\section{Y. Pang}

Brookhaven National Lahoratory, Upton, NY 11973, USA

Received May 1992: editor: D.N. Schramm

\section{Contents:}

1. Introduction

2. Examples of classical nontopological soliton solutions

2.1. Scalar field in one space-dimension

2.2. Scalar field in any space-dimension $D$

2.3. An example in $D=3$

3. Stability and quantization

3.1. General discussion

3.2. Stability of the classical soliton solution 270

3.3. Quantization 270

4. Bose liquid

4.1. General discussion

4.2. Nonrelativistic solitons

4.3. Quantum theory

4.4. Bose hard spheres

4.5. Spontaneous symmetry breaking
5. Friedberg-Lee model for hadron 5.1. General discussion

5.2. Classical solutions

5.3. Mean field approximation

$5 . t$ Beyond mean ficld approximation

6.1. General discussion 3101

6.2. Action of gravitational field

6.3. Action of scalar field

6.4. Equations of motion for besen stars

6.5. Hamiltonian and mass formulae 314

6.6. Boson stars

6.7. Mini-boson stars

6.8. Fermion soliton stars

6.9. Remark

Riferences $3+x$

Abstract:

Nontopological solitons are stable bound state solutions whose boundary condition at infinity is the satne as that for the physical vacuum state. They exist in classical. as well as quantum mechanical, field theories. These solutions in any space-dimension are reviewed. We also discuss the ir applications to Bose liquid, hadron structures and soliton stars.

"This research was supported in part by the US Department of Energy. 


\section{Introduction}

In field theories with an appropriate amount of nonlinearity, stable bound states can exist on a classical, as well as a quantum mechanical, level. These bound states are called solitons. Unlike wave packets formed by superpositions of plane waves, the soliton solutions are nondispersive. They have a finite stable shape in space, and can travel with a constant velocity. Such solutions exist in hydrodynamics, a phenomenon first observed by Russell [1] in the eighteen forties, and later explained in terms of soliton solutions of the nonlinear hydrodynamical equation: the Korteweg-de Vries equation [2]. Since then other equations have been found which also permit soliton-type solutions. Most of these are in one space-dimension, due to a theorem by Derrick [3] which imposes severe restrictions on the types of soliton solutions that can exist for space-dimensions greater than one. In order to have soliton solutions in two or more space-dimensions, one must either include appropriate gauge fields of nonzero spin, or consider time-dependent but nondispersive solutions. In the past few decades, significant progress has been made in the field of solitons, especially those in two or three space-dimensions. If we restrict ourselves to relativistic local field theories, then all soliton solutions can be classified into two general types.

(1) Topological solitons. It is necessary that the boundary condition at infinity for a soliton state should be topologically different from that of the physical vacuum state. In turn, this requires degenerate vacuum states. An example of the topological soliton is the magnetic monopole solution of 't Hooft [4] and Polyakov [5].

(2) Nontopological solitons. The boundary condition at infinity for a non-topological soliton is the same as that for the vacuum state. Thus there is no need for degenerate vacuum states. The necessary condition for the existence of nontopological solitons is that there should be an additive conservation law.

It is the latter, the nontopological soliton, that we will discuss in this review. Earlier examples of this type of solution have been studied by Rosen [6], Kaup [7], Ruffini and Bonazzola [8], Vinciarelli [9], and Lee and Wick [10]. The latter work is also related to the bag models of Chodos et al. [11] and Bardeen et al. [12]. Systematic studies of the subject have been carried out by Friedberg, Lee and Sirlin [13-15] and by others [16]. Soliton solutions containing scalar fields, fermion fields and gauge fields are constructed. Of these, the most thoroughly studied is the Friedberg-Lee [17-19] soliton model for hadrons $[20,21]$.

There is a large amount of literature on nontopological soliton solutions. Most of it involves one or two scalar fields. To introduce nontopological solitons, we shall begin with scalar fields and start our discussion with classical solutions. Section 2.1 studies in detail the one-dimensional case. Despite its simplicity, the one-dimensional soliton shares many features with solitons in higher dimensions. Scalar-field solitons in higher dimensions are given in sections 2.2 and 2.3.

As we shall see, for every classical soliton solution, there is a quantum mechanical counterpart $[22,23]$. In the limit when the nonlinear coupling constant $g$ approaches zero, the quantum soliton solution (times $g$ ) goes to the corresponding classical solution. The quantization of a soliton solution depends on the stability of the classical soliton solution, and can be carried out by using the small perturbation expansion around it. Section 3 deals with the stability analysis and the quantization 
process. Some general discussions are given in section 3.1, stability under small perturbations is shown in section 3.2, and quantization in section 3.3.

It is generally believed that QCD is the correct theory for the strong interaction and that eventually the detailed hadronic structure will be calculated from QCD. In the high energy region, because of asymptotic freedom, perturbation expansion is valid. This enables us to deduce many parameters for the quarks from high energy experiments. But in the low energy region. where it is of importance to the hadronic structure, the theory cannot be solved perturbatively, and nonperturbative effects dominate. The origin of quark confinement remains a major theoretical difficulty. For practical calculations. phenomenological models have been built to take into account some of the nonperturbative effects, so that the rest of the theory can be solved by a perturbative method. As an application of nontopological solitons, section 5 discusses the Friedberg-Lee model. There are two very good reviews on this model and its variations. One is the volume "Nontopological Solitons" by Wilets [20], the other is a review article by Birse [21]. Here we will give a summary of major results.

Another application of nontopological solitons is in exotic stellar structures, called soliton stars [24-27] to be discussed in section 6 . Stable cold stellar configurations have been found which can have masses up to that of a galaxy. Soliton stars can be made either of boson fields or fermion fields. Their characteristics depend sensitively on nonlinear couplings. These stellar configurations all have energies lower than the corresponding free particle (plane wave) solution for any given particle number; consequently, soliton stars are prevented from decaying into free particles. When a soliton star exceeds a certain critical mass, it collapses into a black hole. Because the mechanism for stability is quite different between soliton stars and ordinary stars, the soliton stars can have very different critical masses $[7,8,24,28]$ from the usual Oppenheimer-Volkov [29] type limit.

Recently, the nontopological soliton has been extended to the study of the Bose liquid [30]. The liquid-gas phase transition can be formulated in terms of the spontaneous symmetry breaking of translational invariance in the canonical ensemble, while the Bose-Einstein transition is due to the breaking of the phase symmetry of the complex scalar field in its grand canonical average. The former can describe the liquid-gas phase transition of helium, and the latter the $\Lambda$ transition between HeI and HeII. These will be discussed in section 4 (because its methodology parallels that given in section 3 ).

Throughout the paper we adopt the natural units $\hbar=c=1$.

\section{Examples of classical nontopological soliton solutions}

\subsection{Scalar field in one space-dimension $|31,32|$}

The simplest system which has nontopological soliton solutions is a complex scalar field in one space-dimension (plus the time-dimension). For a flat space-time $x^{\mu}=(t, x)$ with $\mu=0$ or 1 .

$$
\left(g_{\mu \prime}\right)=\left(\begin{array}{rr}
-1 & 0 \\
0 & 1
\end{array}\right), \quad\left(g^{\mu u^{\prime}}\right)=\left(g_{\mu \nu}\right)^{\prime} .
$$

The requirement of an additive conservation law can be met by having the gauge invariance under an arbitrary constant phase transformation,

$$
\phi \rightarrow \mathrm{e}^{-i+\mu} \phi \text {. }
$$


The Lagrangian density is assumed to be

$$
\mathscr{L}=-\phi^{\dagger \mu} \phi_{\mu}-U\left(\phi^{\dagger} \phi\right)
$$

where $\phi^{+}$is the Hermitian conjugate of $\phi$,

$$
\phi_{\mu}=\partial \phi / \partial x^{\mu}, \quad \phi_{\mu}^{\dagger}=\partial \phi^{\dagger} / \partial x^{\mu}, \quad \phi^{\mu}=g^{\mu \nu} \phi_{\nu}, \quad \phi^{\dagger \mu}=g^{\mu \nu} \phi_{\nu}^{\dagger} .
$$

The equation of motion is

$$
\partial \phi^{\mu} / \partial x^{\mu}-\phi \mathrm{d} U\left(\phi^{\dagger} \phi\right) / \mathrm{d}\left(\phi^{\dagger} \phi\right)=0 \text {. }
$$

Hence the current $j^{\mu}$, defined by

$$
j^{\mu} \equiv-\mathrm{i}\left(\phi^{\dagger} \phi^{\mu}-\phi^{\dagger \mu} \phi\right)
$$

is conserved:

$$
\partial j^{\mu} / \partial x^{\mu}=0
$$

The particle density is $j^{0}$. Its space integral is the particle number $N$,

$$
N=\int j^{0} \mathrm{~d} x
$$

Because of current conservation (2.7), $N$ is conserved, i.e. the time derivative

$$
\dot{N}=0 \text {. }
$$

In the following, the volume of the system is taken to be infinite, so that $x$ varies from $-\infty$ to $\infty$.

The potential function $U\left(\phi^{\dagger} \phi\right)$ is assumed to have a single absolute minimum at $\phi=0$; the minimum value can always be chosen to be zero. Thus, as $\phi \rightarrow 0$, we have

$$
U \rightarrow m^{2} \phi^{\dagger} \phi
$$

with the mass parameter $m>0$. Later, in the quantum theory, this implies a nondegenerate vacuum state; therefore, the gauge symmetry (2.2) is not broken spontaneously by the physical vacuum state. (However, for macroscopic states with a nonzero density $N / \Omega$, when $N \rightarrow \infty$ spontaneous symmetry breaking may appear, as we shall discuss in section 4.3.)

From (2.6)-(2.8) it follows that for $N \neq 0, \phi$ must vary with time. As we shall see, at a fixed particle number $N$ the time dependence of $\phi$ for the lowest energy solution must be of the form

$$
\phi \propto \mathrm{e}^{-\mathrm{i} \omega t} .
$$

To establish this, write 


$$
\phi=\left(\phi_{\mathrm{R}}+\mathrm{i} \phi_{1}\right) / \sqrt{2}
$$

where $\phi_{\mathrm{R}}$ and $\phi_{1}$ are both real. Denote

$$
\dot{\phi}_{\mathrm{R}}=\dot{\partial} \phi_{\mathrm{R}} / \dot{\partial} t, \quad \dot{\phi}_{\mathrm{I}}=\dot{d} \phi_{\mathrm{I}} / \dot{\partial} t
$$

Substituting the above into the equations for $N,(2.6)$ and (2.8), we have

$$
N=\int\left(\phi_{\mathrm{I}} \dot{\phi}_{\mathrm{R}}-\phi_{\mathrm{R}} \dot{\phi}_{1}\right) \mathrm{d} x
$$

The energy of the system is

$$
E=\int\left\{\frac{1}{2}\left[\dot{\phi}_{\mathrm{R}}^{2}+\dot{\phi}_{1}^{2}+\left(\mathrm{d} \phi_{\mathrm{R}} / \mathrm{d} x\right)^{2}+\left(\mathrm{d} \phi_{\mathrm{I}} / \mathrm{d} x\right)^{2}\right]+U\left[\frac{1}{2}\left(\phi_{\mathrm{R}}^{2}+\phi_{\mathrm{I}}^{2}\right)\right]\right\} \mathrm{d} x .
$$

At any given time $t$, assuming that $\phi_{\mathrm{R}}, \phi_{1}$ and $N$ are given, we wish to find the $\dot{\phi}_{\mathrm{R}}$ and $\dot{\phi}_{1}$ which make $E$ minimal. Consider an infinitesimal variation.

$$
\dot{\phi}_{\mathrm{R}} \rightarrow \dot{\phi}_{\mathrm{R}}+\delta \dot{\phi}_{\mathrm{R}}, \quad \dot{\phi}_{\mathrm{I}} \rightarrow \dot{\phi}_{1}+\delta \dot{\phi}_{1}
$$

keeping $\phi_{\mathrm{R}}$ and $\phi_{\mathrm{I}}$ fixed. The minimum energy solution is determined by

$$
\delta(E-\omega N)=()
$$

where $\omega$ is the Lagrange multiplier. This leads to

$$
\dot{\phi}_{\mathrm{R}}=\omega \phi_{\mathrm{I}}, \quad \dot{\phi}_{\mathrm{I}}=-\omega \phi_{\mathrm{R}},
$$

and therefore

$$
\phi=\sigma(x) \mathrm{e}^{-i \omega t},
$$

where $\sigma(x)$ can be set to be real. The procedure above can easily be generalized to higher space-dimensions and to more sophisticated systems (like scalar soliton star solutions in general relativity).

In terms of $\sigma$, the equation of motion (2.5) becomes

$$
\mathrm{d}^{2} \sigma / \mathrm{d} x^{2}+\omega^{2} \sigma-\sigma \mathrm{d} U\left(\sigma^{2}\right) / \mathrm{d} \sigma^{2}=0
$$

which, after being multiplied by $\mathrm{d} \sigma / \mathrm{d} x$, can be integrated. The result is

$$
\frac{1}{2}(\mathrm{~d} \sigma / \mathrm{d} x)^{2}-V(\sigma)=\text { constant }
$$

where

$$
V(\sigma)=\frac{1}{2} U\left(\sigma^{2}\right)-\frac{1}{2} \omega^{2} \sigma^{2} .
$$


In accordance with (2.9), the absolute minimum of $U\left(\sigma^{2}\right)$ is at $\sigma=0$, with

$$
U \rightarrow m^{2} \sigma^{2}, \text { as } \sigma \rightarrow 0
$$

In order to have nontopological solitons, the function $V$ defined by $(2.20)$ must be of the form given by fig. 1. More specifically, the condition

$$
U\left(\sigma^{2}\right)-\omega^{2} \sigma^{2}=0
$$

has $\sigma \neq 0$ solutions for some $\omega^{2}$ less than $m^{2}$. The same condition may also be stated alternatively in terms of the difference

$$
v\left(\sigma^{2}\right) \equiv U\left(\sigma^{2}\right)-m^{2} \sigma^{2}
$$

which denotes the nonlinear interaction. If for any range of $\sigma$ however small, $v$ is attractive $(i . e . v<0)$ however weakly, then solitons exist. To see the equivalence, we note that the latter condition, together with (2.9) and the positivity of $U$, implies that there exists a parabola $\nu^{2} \sigma^{2}$ tangent to $U\left(\sigma^{2}\right)$ at $\sigma= \pm \sigma_{0}$, with $\nu^{2}<m^{2}$, as shown in figs. 2a and $2 \mathrm{~b}$. Hence, for

$$
\nu^{2} \leq \omega^{2}<m^{2}
$$

the parabola $\omega^{2} \sigma^{2}$ must intercept $U\left(\sigma^{2}\right)$, i.e. $V(\sigma)=0$ has $\sigma \neq 0$ solutions.

To derive the soliton solution in one space-dimension, consider a simple mechanical analog, in which a point particle is moving in a potential $-V(\sigma)$, with $\sigma$ as its "position" and $x$ its "time", as shown in fig. 3. Then (2.19) becomes simply the energy conservation law in the analog problem. At the "time" $x=-\infty$, set the particle at the "position" $\sigma=0$. We may start the motion by an extremely gentle push towards the right. As $x$ increases, $\sigma$ moves to $A$ and then returns to 0 at $x=+\infty$. The general solution is given by

$$
x-\xi=\int_{A}^{\sigma} \frac{\mathrm{d} \sigma}{\sqrt{2 V(\sigma)}},
$$

where $\xi$ is the integration constant. The problem of finding any one-space-dimensional soliton solution

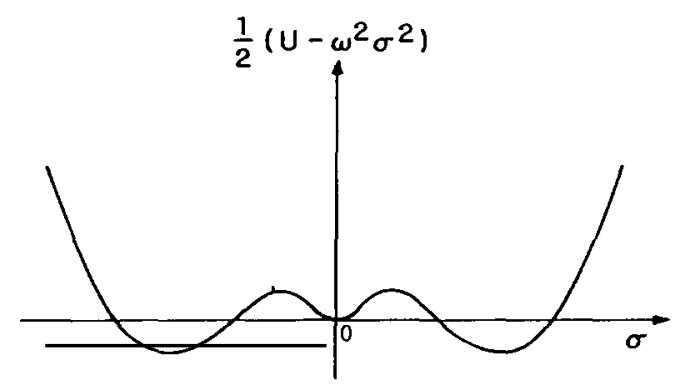

Fig. 1. The function $\frac{1}{2}\left(U-\omega^{2} \sigma^{2}\right)$ versus $\sigma$ for $\omega^{2}<m^{2}$. 

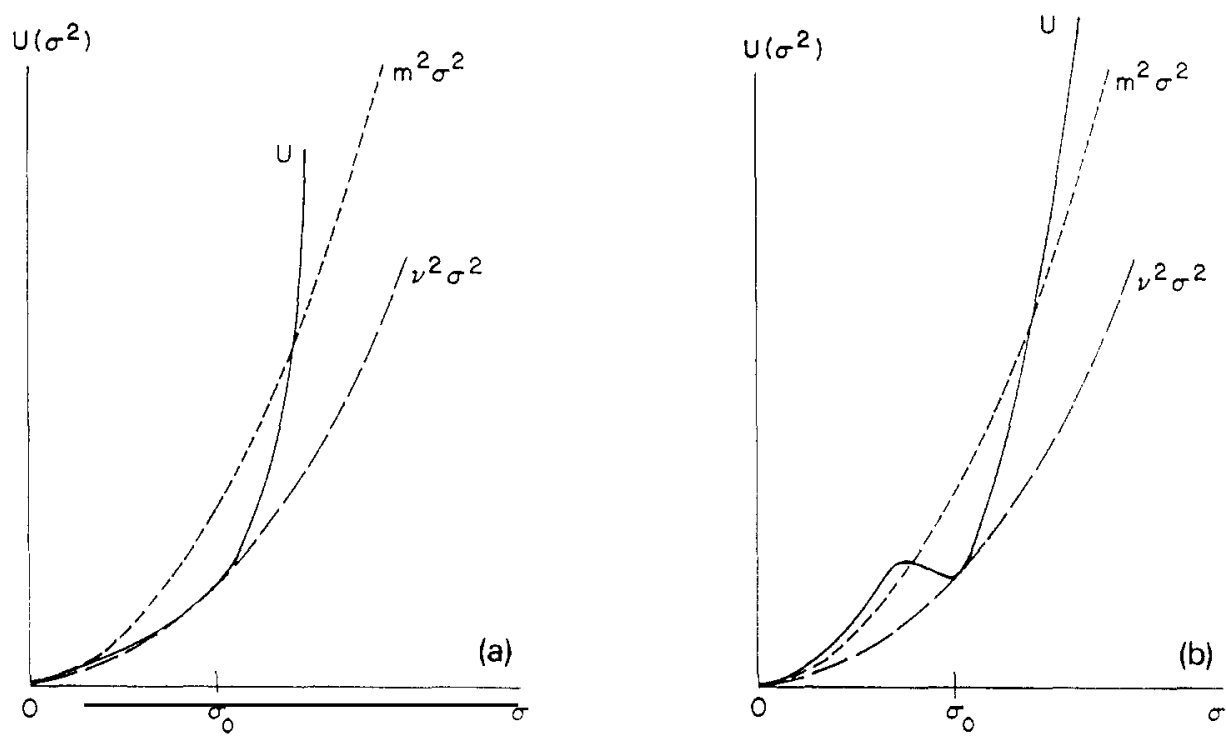

Fig. 2. $U\left(\sigma^{2}\right)$ versus $\sigma$. For $\sigma$ small and $>0$. (a) $U<m^{2} \sigma^{2}$ and (b) $U>m^{2} \sigma^{2}$. The parabola $\nu^{2} \sigma^{2}$ is tangent to $U$ at $\sigma=\sigma_{t}$ in both cases.

is reduced to quadrature. A schematic drawing of the solution is given in fig. 4 . When $x=\xi, \sigma=A$. At both infinities, $x \rightarrow+\infty$ or $-x, \sigma$ satisfies the same boundary condition:

$$
\sigma \rightarrow 0
$$

Therefore, it represents a nontopological soliton solution.

When $\sigma \rightarrow 0$, the function $V$ becomes

$$
V \rightarrow \frac{1}{2}\left(m^{2}-\omega^{2}\right) \sigma+\mathrm{O}\left(\sigma^{4}\right)
$$

which, for $\omega^{2}<m^{2}$, makes $V(\sigma)$ concave upward at the origin $\sigma=0$. Also, when $|x|$ is large, $\sigma$ is small; from (2.19) we see that $\sigma$ must decay exponentially, as

$$
\sigma \sim \exp \left(-\sqrt{m^{2}-\omega^{2}}|x|\right) .
$$

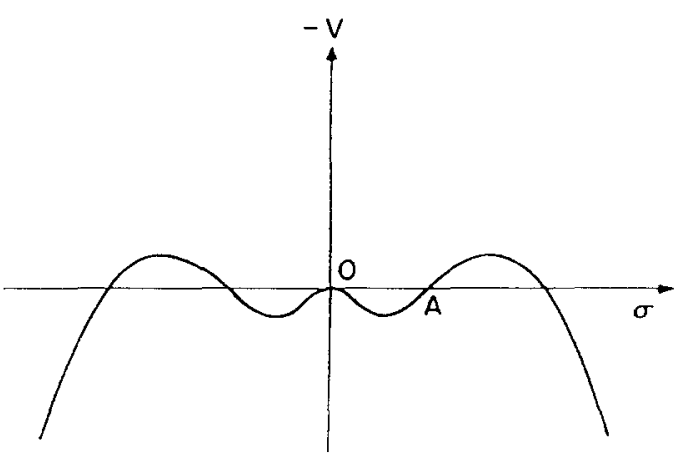

Fig. 3. $-V$ versus $\sigma$ for $\omega^{2}<m^{2}$

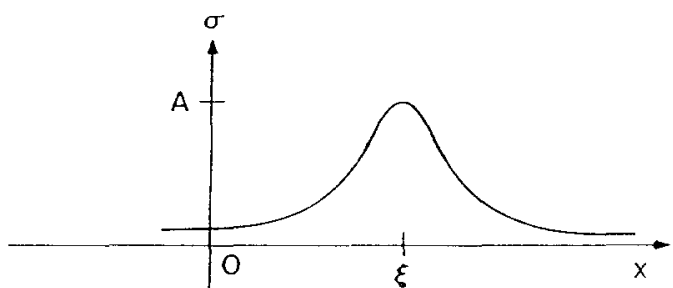

Fig. 4. The soliton solution (2.23). 
Condition (2.21) can be most easily satisfied if the $O\left(\sigma^{4}\right)$ term in $V$ is negative. (Of course, some higher power terms must be positive to make the potential $U$ always positive.) In this case, when $\omega \rightarrow m-$, the position of the zero $\sigma=A$ of $V(\sigma)=0$ must be near the origin; it is determined by the quadratic and quartic terms in $V$, which leads to $A^{2} \propto\left(m^{2}-\omega^{2}\right) \rightarrow 0$. Consequently, we can improve the asymptotic form $(2.26)$, and write

$$
\sigma \sim\left(m^{2}-\omega^{2}\right)^{1 / 2} \exp \left(-\sqrt{m^{2}-\omega^{2}}|x|\right)
$$

(Under the same assumption of $V$, this behavior can be generalized to any space-dimension, as will be given by (2.58).)

The simplest polynomial form of $U$ which permits a nontopological soliton solution is cubic in $\phi^{\dagger} \phi$ :

$$
U=\left[m^{2} \phi^{\dagger} \phi /\left(1+\varepsilon^{2}\right)\right]\left[\left(1-g^{2} \phi^{\dagger} \phi\right)^{2}+\varepsilon^{2}\right] .
$$

The solution is

$$
\phi=g^{-1}[a /(1+\sqrt{1-a} \cosh y)]^{1 / 2} \mathrm{e}^{-\mathrm{i} \omega t},
$$

where

$$
\begin{aligned}
& a=\left(1+\varepsilon^{2}\right)\left(m^{2}-\omega^{2}\right) / m^{2}, \\
& y=2 \sqrt{m^{2}-\omega^{2}}(x-\xi) .
\end{aligned}
$$

Because the parameter $\xi$ is a constant, the solutions (2.23) and (2.29)-(2.31) represent solitons at rest. The relativistic invariance of the theory ensures the generalization to solitons with an arbitrary momentum $\boldsymbol{P}$. The parameter $g$ is a measure of the nonlinearity of the system. When $g=0$, the potential energy $U$ is quadratic in $\phi$, and the equation of motion becomes linear; only plane wave solutions exist. When $g$ is nonzero, one has soliton solutions (2.23) and (2.29). As $g \rightarrow 0$, the soliton amplitude grows as $1 / \mathrm{g}$. As we shall show, this is an important and general feature of soliton solutions (both topological and nontopological): the existence of solitons does not depend on the strength of the nonlinear coupling $g$, so long as $g \neq 0$; all soliton solutions are singular as the nonlinear coupling $g \rightarrow 0$. Consequently,

weak coupling $\neq$ weak amplitude .

In classical theory this singularity is always a simple pole: for any function $U$ of $\phi^{\dagger} \phi$, we can introduce $\bar{U}$ by writing

$$
U\left(\phi^{\dagger} \phi\right) \equiv g^{-2} \bar{U}\left(g^{2} \phi^{\dagger} \phi\right) .
$$

Define $\bar{\phi}$ by

$$
\phi=g^{-1} \bar{\phi} .
$$


Then $g$ can be factored out of our Lagrangian density (2.3)

$$
\mathscr{L}=g^{-2 \overline{\mathscr{L}}}
$$

where

$$
\overline{\mathscr{L}}=-\bar{\phi}^{\mu} \bar{\phi}_{\mu}-\bar{U}(\bar{\phi} \bar{\phi})
$$

is $g$-independent. Thus the solution $\bar{\phi}$ is also $g$-independent.

It is obvious that (for any finite $N$ ), when the volume $\Omega$ that encloses the whole system approaches infinity, the equation of motion (2.5) admits the usual plane-wave solution

$$
\phi=\sqrt{N / 2 \omega \Omega} \mathrm{e}^{i(k x-\omega t)}
$$

where

$$
\omega=\sqrt{k^{2}+m^{2}}
$$

This follows from the fact that, as $\Omega \rightarrow x$, the amplitude $\phi$ is infinitesimal, so the equation of motion becomes linear in $\phi$.

The soliton solution differs from the plane wave solution, since its amplitude does not become infinitesimal as $\Omega \rightarrow \infty$. Furthermore, when $x \rightarrow \pm \infty$, the soliton amplitude approaches zero exponentially, with $\omega^{2}<m^{2}$. We may regard these two types of solution as analytical continuations of each other:

$$
\omega^{2}>m^{2} \text { for the plane wave solution, } \omega^{2}<m^{2} \text { for the soliton solution }
$$

If we plot the energy $E$ versus the particle number $N$ (taken to be positive, for simplicity), as in fig. 5 , we see a straight line for the plane wave solutions of zero momentum, with a slope $m$, and a convex curve for the soliton solutions, with its slope at every point given by

$$
\mathrm{d} E / \mathrm{d} N=\omega
$$

This is actually just one of the Hamiltonian equations for the system. Since $N$ and the phase angle

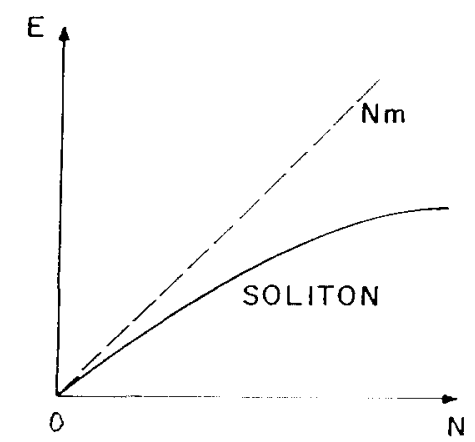

Fig. $5 . E$ versus $N$ in one space dimension. 
$\theta=\omega t$ are conjugate variables, we have

$$
\dot{N}=-\partial H / \partial \theta, \quad \dot{\theta}=\partial H / \partial N
$$

where $H$ is the Hamiltonian. Because of the invariance under the phase transformation (2.2), the Hamiltonian is independent of $\theta$. Therefore, the first equation is $\dot{N}=0$, i.e. the conservation of $N$. The second equation gives (2.39).

As $\omega^{2} \rightarrow m^{2}-$, from $(2.27)$,

$$
N=\int 2 \omega \sigma^{2} \mathrm{~d} x \sim \sqrt{m^{2}-\omega^{2}} \rightarrow 0 .
$$

Since the nontopological soliton solution is the analytic continuation of the plane-wave solution to the region $\omega^{2}<m^{2}$, the curve $E$ (soliton) versus $N$ should be connected to the straight line $E$ (plane wave $=|N| m$, where $\omega^{2} \rightarrow m^{2}-$. From (2.40) we see that this connection occurs at $N=0$.

In fig. 3 , as we reduce $\omega^{2}$, the maximum of $-V$ becomes smaller. When $\omega^{2} \rightarrow \nu^{2}+$, in accordance with (2.21)-(2.22), the $-V>0$ peak is just infinitesimally above zero, as shown in fig. 6. We can re-examine our previous mechanical analog: the "particle" leaves point $\mathrm{O}$ at "time" near $-\infty$ with a "velocity" close to zero. When it reaches A, which is almost near the peak, at some finite "time", instead of turning back immediately it can stay near $\sigma=\sigma_{0}$ for a very long "time", say $L$. As in figs. 2a and $2 \mathrm{~b}$, the value $\sigma_{0}$ denotes the point when the tangent parabola $\nu^{2} \sigma^{2}$ touches $U\left(\sigma^{2}\right)$; hence, at $\omega^{2}=\nu^{2},-V=-U+\nu^{2} \sigma^{2}$ is tangent to the abscissa at $\sigma=\sigma_{0}$. The corresponding soliton has a very large $N$; its shape is illustrated in fig. 7. Choose $\nu$ to be positive, as $\omega \rightarrow \pm \nu$,

$$
N \simeq \pm 2 \nu \sigma_{0}^{2} L
$$

where $L$, the size of the soliton solution, may approach $\infty$. From (2.39), we see that for $|N| \gg 1$, neglecting $\mathrm{O}(1)$, the soliton energy $E$ is

$$
E=\nu|N| \text {. }
$$

In one space-dimension, because $E \rightarrow m|N|$ when $N \rightarrow 0$, the integration of (2.39) gives the soliton

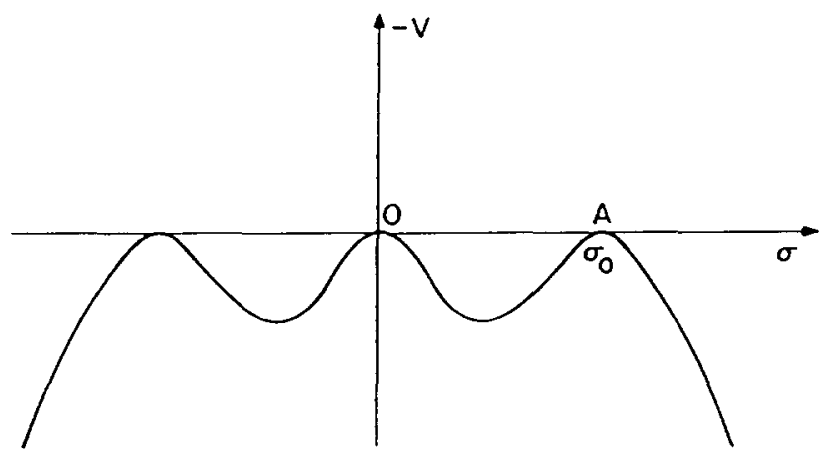

Fig. 6. $-V$ versus $\sigma$ when $\omega^{2} \rightarrow v^{2}+$ 


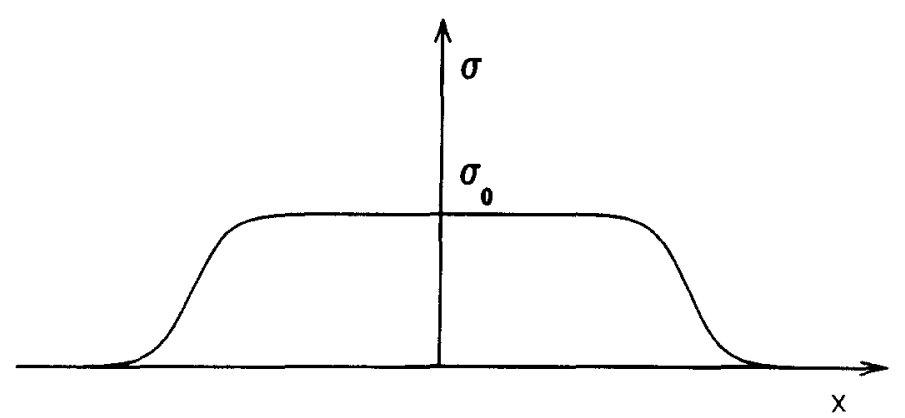

Fig. 7. As $\omega^{\prime \prime} \rightarrow v^{2}+$, the soliton size becomes larger and larger.

energy $E$ for any $N \neq 0$. We find

$$
0<E=\int_{0}^{N} \omega \mathrm{d} N<m|N| .
$$

Hence, if the theory admits a nontopological soliton solution, then soliton solutions exist for all particle numbers $N$. Furthermore, the solitons always have lower energy than the plane wave solutions of the same $N$. (However, this statement has to be modified in higher space-dimensions, as we shall see in the next section.)

\subsection{Scalar field in any space-dimension $D$}

In this section, we extend the classical nontopological soliton solutions for a complex scalar field to any space-dimension $D$. The Lagrangian density remains given by $(2.3)$ :

$$
\mathscr{L}=-\phi^{\dagger \mu} \phi_{\mu}-U\left(\phi^{\dagger} \phi\right)
$$

but the index $\mu$ now varies from $0,1 \ldots$ to $D$, with $x^{\prime \prime}=t$. Equations (2.2)-(2.17) are all valid, provided one replaces the one-dimensional space-coordinate $x$ by a $D$-dimensional space-vector $r$, the differential $\mathrm{d} x$ by $\mathrm{d}^{D} r$, and the gradient $\partial / \partial x$ by $\boldsymbol{\nabla}$. Thus, as in (2.17), at a given particle number $N$ the lowest energy solution must be of the form

$$
\phi=\sigma(\boldsymbol{r}) \mathrm{e}^{-i \omega t}
$$

where $\sigma(r)$ can be set to be real.

In terms of $\sigma$, the particle number $N$ is given by

$$
N=2 \omega \int \sigma^{2} \mathrm{~d}^{D} r
$$

and the energy by

$$
E=\int\left[\omega^{2} \sigma^{2}+(\boldsymbol{\nabla} \sigma)^{2}+U\left(\sigma^{2}\right)\right] \mathrm{d}^{D} r
$$


The equation of motion is

$$
\nabla^{2} \sigma+\omega^{2} \sigma-\sigma \mathrm{d} U\left(\sigma^{2}\right) / \mathrm{d} \sigma^{2}=0
$$

which can also be derived by keeping $N$ fixed and setting the variational derivative $(\delta E / \delta \sigma)_{N}=0$. As before, the absolute minimum of $U$ is at $\sigma=0$,

$$
U \rightarrow m^{2} \sigma^{2} \text { as } \sigma \rightarrow 0
$$

and

$$
v \equiv U-m^{2} \sigma^{2}
$$

represents the nonlinear interaction. The volume $\Omega$ of the system is set to be $\infty$.

Theorem. In any space-dimension $D$, if there exists a range of $\sigma$ however small, and the potential function $U\left(\sigma^{2}\right)$ contains any attractive interaction (i.e. $v<0$ ) however weak, then nontopological soliton solutions exist for

$$
\nu^{2} \leq \omega^{2}<m^{2},
$$

where, as in figs. $2 \mathrm{a}$ and $2 \mathrm{~b}$, when plotted versus $\sigma$ the parabola $\nu^{2} \sigma^{2}$ is tangent to $U\left(\sigma^{2}\right)$ at

$$
\sigma=\sigma_{0}
$$

For $N$ sufficiently large, neglecting $\mathrm{O}(1)$, the lowest energy soliton solution has an energy

$$
E=|\nu N|<m|N|
$$

which ensures its stability against decaying into plane wave solutions. Furthermore, except near its surface, the amplitude $\sigma$ is essentially a constant

$$
\sigma \simeq \sigma_{0}
$$

inside and $\sigma \simeq 0$ outside.

Proof [30]. We first establish that the lowest energy solution $\sigma$ has no node. Since the solution $\sigma$ of (2.47) has no singularity, for any $\sigma$ with nodes, the $(D-1)$ dimensional nodal surface $\mathscr{I}$, defined by $\sigma=0$, must form (at least) one closed surface (or one that extends to infinity), with $\sigma$ of opposite signs across $\mathscr{Y}$. Change $\sigma \rightarrow+\sigma$ on one side of $\mathscr{Y}$ and $\sigma \rightarrow-\sigma$ on the other; this leaves the integrals (2.45) and (2.46) for $N$ and $E$ unaltered. The new $\sigma$ has a cusp on $\mathscr{S}$; therefore, its energy $E$ can be reduced by smoothing the cusp, which makes the original $\sigma$ (with a nodal surface) not of the lowest energy. In the following, we consider the spherically symmetric soliton solution with no radial node. (Using the discussion given by Coleman [16], one can give a general proof that, for large $N$, such a zero-node spherically symmetric soliton solution is indeed the absolute minimum of the Hamiltonian.) 
By using the phase transformation (2.2), we choose $\sigma(r)$ to be positive (where $r=|r|$ ). Equation (2.47) becomes

$$
\frac{\mathrm{d}^{2} \sigma}{\mathrm{d} r^{2}}+\omega^{2} \sigma-\sigma \frac{\mathrm{d} U\left(\sigma^{2}\right)}{\mathrm{d} \sigma^{2}}=-\frac{D-1}{r} \frac{\mathrm{d} \sigma}{\mathrm{d} r} .
$$

i.e.

$$
\frac{\mathrm{d}}{\mathrm{d} r}\left[\frac{1}{2}\left(\frac{\mathrm{d} \sigma}{\mathrm{d} r}\right)^{2}-\overline{V(\sigma)}\right]=-\frac{D-1}{r}\left(\frac{\mathrm{d} \sigma}{\mathrm{d} r}\right)^{2} .
$$

where, as in $(2.20)$,

$$
V(\sigma)=\frac{1}{2} U\left(\sigma^{2}\right)-\frac{1}{2} \omega^{2} \sigma^{2}
$$

Because under $\omega \rightarrow-\omega, N \rightarrow-N$ but $E$ is unchanged, we need only examine the region where $\omega$ and $N$ are both positive.

It is useful to consider the same mechanical analog problem discussed in the previous section, with $\sigma$ and $r$ being the "position" and the "time" of a point particle, which moves in a "potential" $-V(R)$. The right-hand side of (2.54) is zero for $D=1$, but always negative for $D>1$. Therefore, for $D=1$ the total particle "energy" $\frac{1}{2}(\mathrm{~d} \sigma / \mathrm{d} r)^{2}-V(\sigma)$ is conserved, but for $D>1$ we have a dissipative system in the analog problem.

Choose $\nu$ positive. In the limit $\omega \rightarrow \nu+,-V$ is given by fig. 6. For $D>1$, we set the particle at $\sigma=\sigma_{0}-$ at $r=0$. Because, when $\omega=\nu,-V$ is tangent to the abscissa at $\sigma=\sigma_{0}$, the particle can be nearly stationary at its initial "position" $\sigma_{0}$ for a very long "time", $r=R$. By then, the dissipation can be made extremely small, because it is proportional to $r^{-1}$. Hence, one can always find a solution: $\sigma \simeq \sigma_{0}$ for $0 \leq r \leq R$ then decreases to $\sigma=0$ at $r=x$. For $R$ sufficiently large, the transition is approximately given by

$$
r \simeq R+\int_{\sigma}^{\sigma_{11}} \frac{\mathrm{d} \sigma}{\sqrt{2 V(\sigma)}} .
$$

Because of (2.39), $\mathrm{d} E / \mathrm{d} N=\omega$, for this type of very large $N$ solutions (with $\omega=v^{\prime-}$ )

$$
E=\nu N+\text { remainder } .
$$

For a macroscopic $N$, the volume of the soliton is $\sim N / 2 \nu \sigma_{0}^{2}$. Inside the volume the matter density $\sigma^{2}$ is nearly a constant, $\sigma_{0}^{2}$. Near the soliton radius $R, \sigma$ changes rapidly from $\sigma \simeq \sigma_{0}$ to $\sigma \simeq 0$ in accordance with (2.56), giving rise to a surface energy $\mathrm{O}\left(N^{(D-1) D}\right)$ which is the remainder. We may write, as $\omega=\nu-, N \rightarrow \infty$,

$$
E \rightarrow \nu N+s N^{(D-1) ! D}
$$

with the constant $s>0$. Equation (2.52) of the theorem is then established. 
As we increase $\omega$ from $\nu+$ (but less than $m$ ), the $-V>0$ peak in fig. 3 rises steadily. If we set $\sigma$ near the peak at the initial "time" $r=0, \sigma$ can again be almost stationary until a very long "time" $r=R$, whereupon by neglecting the dissipation in (2.54) we see that $\sigma$ would reach $\sigma=0$ at a finite $r>R$. Consequently, by moving the initial "position" $\sigma$ at $r=0$ towards the origin, there must exist a solution of (2.54) that reaches $\sigma=0$ at $r=\infty$, which proves the theorem.

As already mentioned in the previous section, in any space-dimension $D$ the nontopological soliton solution is the analytic continuation of the plane wave solution to the region $\omega<m$. Again, consider only the case $N>0$ (therefore, $\omega>0$ ). On an $E$ versus $N$ diagram, the curve representing the soliton solution should be connected to the straight line $E=N m$ of the plane wave solution when $\omega \rightarrow m-$. The connecting point depends sensitively on $D$, as we shall see. Assuming that the quartic term in $U$ is negative, then at large $r$ and for $\omega \rightarrow m-,(2.27)$, or its generalization

$$
\sigma \sim\left(m^{2}-\omega^{2}\right)^{1 / 2} \exp \left(-\sqrt{m^{2}-\omega^{2}} r\right)
$$

holds. By using (2.45), we see that as $\omega \rightarrow m-$

$$
N \rightarrow 2 m \int \sigma^{2} \mathrm{~d}^{D} r=\left\{\begin{array}{cc}
0, & \text { for } D=1, \\
\mathrm{O}(1), & \text { for } D=2, \\
\infty, & \text { for } D \geq 3
\end{array}\right.
$$

because $\int \sigma^{2} \mathrm{~d}^{D} r \sim\left(m^{2}-\omega^{2}\right) \times\left(m^{2}-\omega^{2}\right)^{-D / 2}$. The typical $E$ (soliton) versus $N$ curves for these three cases are given in figs. 5,8 and 9 .

The $D \geq 3$ case has a more intriguing shape. It is not difficult to show that when $\omega=m-, N \rightarrow \infty$, $E \sim N m$ but the difference $E$ (soliton) $-N m$ is infinitesimally positive. As $\omega$ decreases from $m-, N$ and $E$ both decrease in accordance with $\mathrm{d} E / \mathrm{d} N=\omega$ (still with $E$ (soliton) $>N m$ ) until $N=N_{\mathrm{C}}$, as shown in fig. 9. When $\omega$ is decreased further, then $N$ and $E$ both start to increase until the $E$ (soliton) versus $N$ curve crosses the straight line $N m$ at $N=N_{\mathrm{s}}$. For $N>N_{\mathrm{S}}, E$ (soliton) is $<N m$. As $\omega \rightarrow \nu+, N$ again $\rightarrow \infty$, but with $E$ (soliton) $\rightarrow N \nu<N m$.

There exists besides a critical point $\mathrm{S}$ (denoting stability) also a cusp C, with $N_{\mathrm{S}}>N_{\mathrm{C}}$. For $N<N_{\mathrm{C}}$, there is no soliton solution. For $N_{\mathrm{C}}<N<N_{\mathrm{S}}$, the lowest-energy solution is the plane wave; for $N>N_{\mathrm{S}}$,

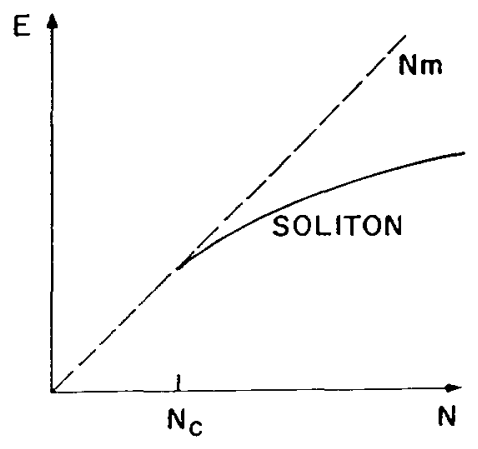

Fig. 8. $E$ versus $N$ in two space dimensions.

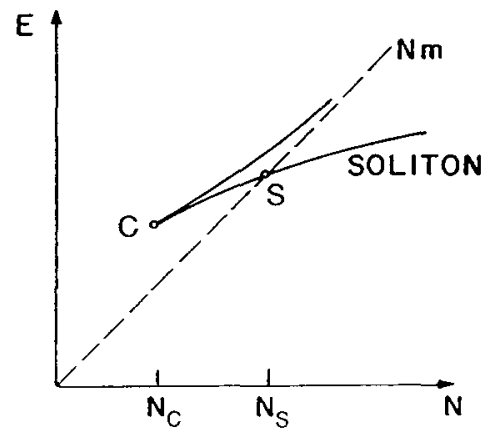

Fig. 9. $E$ versus $N$ in three space dimensions. On the lower branch, for $N_{\mathrm{c}}<N<N_{\mathrm{s}}$, the soliton solution is classically stable against small perturbations, but quantum mechanically metastable. For $N>N_{\mathrm{s}}$, the soliton solution is stable classically as well as quantum mechanically. 
the lowest-energy solution is always the soliton. In fig. 9, along the lower branch of the soliton curve. for $N>N_{\mathrm{S}}$, the soliton solution is absolutely stable, being the lowest-energy solution; it cannot decay into plane wave solutions; because of the curvature $\partial^{2} E / \partial N^{2}=\partial \omega / \partial N<0$, it is also stable against fission. For $N_{\mathrm{S}}>N>N_{\mathrm{C}}$, it can be shown [13] that the soliton solution is stable against infinitesimal perturbations, even though it is not of the lowest energy. Along the upper branch, the soliton solution is always unstable. The numerical values of $N_{\mathrm{S}}$ and $N_{\mathrm{C}}$ depend on the parameters in the theory.

As mentioned in the previous section, if there is only one complex scalar field $\phi$, in order to have both $U$ positive and also some attraction, $U\left(\phi^{\dagger} \phi\right)$ must contain either $\left(\phi^{+} \phi\right)^{3}$ or other higher power terms in $\phi^{\dagger} \phi$. For $D \geq 3$, such a quantum theory is unrenormalizable; to have renormalizability, there must exist other fields besides $\phi$. This will be illustrated by the following $D=3$ example. As will be discussed, its $E$ (soliton) versus $N$ curve also exhibits the same typical shape in fig. 9.

\subsection{An example in $D=3$}

Consider a system consisting of two spin-0 fields, a complex field $\phi$ and a Hermitian field $\chi$. The Lagrangian density $\mathscr{L}$ is assumed to be

$$
\mathscr{L}=-\phi^{\dagger \mu} \phi_{\mu}-\frac{1}{2} \chi^{\mu} \chi_{\mu}-f^{2} \chi^{2} \phi \phi-u(\chi)
$$

where $\phi^{\mu}$ and $\phi_{\mu}$ are defined by the four-dimensional generalization of (2.4). Similarly,

$$
\chi_{\mu}=\partial \chi / \partial x^{\mu}, \quad \chi^{\mu}=g^{\mu \nu} \chi_{\mu}
$$

with $g^{(0)}=-1, g^{11}=g^{22}=g^{33}=1$ and other $g^{\mu \prime}=0$. The potential $u(\chi)$ is a fourth-order polynomial of $\chi$ satisfying $u(x)=\infty$, so that the theory is stable and renormalizable. This example gives the simplest renormalizable model in $D=3$ which permits nontopological soliton solutions. Because of the $\mathrm{U}(1)$ symmetry,

$$
\phi \rightarrow \mathrm{e}^{-\mathrm{i} \theta} \phi
$$

there is the current conservation (2.7) and, consequently, the particle number $N$ defined by (2.8) is a constant of the motion. Assume $u(\chi)$ has an absolute minimum at $\chi=\chi_{v a c}$, its vacuum expectation value. For convenience, we choose $u\left(\chi_{\text {vac }}\right)=0$, and therefore

$$
u(\chi) \geq u\left(\chi_{\text {vac }}\right)=0
$$

The mass of the $\phi$ meson is

$$
m=f \chi_{\text {vac }}
$$

which will be assumed to be nonzero. Thus, $f \neq 0$ and

$$
\chi_{\mathrm{vac}} \neq 0
$$


To simplify further, take

$$
u(\chi)=\frac{1}{8} g^{2}\left(\chi^{2}-\chi_{\mathrm{vac}}^{2}\right)^{2}
$$

so that in addition to the U(1) symmetry, there is a discrete symmetry

$$
\chi \rightarrow-\chi
$$

The details of this model and its generalization are given in ref. [13].

Introduce

$$
\begin{aligned}
& \chi(\boldsymbol{r}, t) \equiv \chi_{\mathrm{vac}} A(\boldsymbol{\rho}) \\
& \phi(\boldsymbol{r}, t) \equiv(1 / \sqrt{2}) \chi_{\mathrm{vac}} B(\boldsymbol{\rho}) \mathrm{e}^{-\mathrm{i} \omega t},
\end{aligned}
$$

where $A$ and $B$ are both dimensionless and real,

$$
\boldsymbol{\rho} \equiv \mu \boldsymbol{r}, \quad \mu=g \chi_{\mathrm{vac}},
$$

where $\mu$ is the mass of the neutral $\chi$ meson. From the Lagrangian density (2.60) and the definitions for $A$ and $B$, the equations of motion follow:

$$
\nabla^{2} A-\kappa^{2} B^{2} A-\frac{1}{2}\left(A^{2}-1\right) A=0, \quad \nabla^{2} B-\kappa^{2} A^{2} B+\nu^{2} B=0
$$

where $\boldsymbol{\nabla}$ is the gradient operator with respect to the dimensionless parameter $\boldsymbol{\rho}$,

$$
\nu \equiv \omega / \mu, \quad \kappa \equiv m / \mu
$$

The parameters $\omega, m$ and $\mu$ can all be taken to be positive. The particle number $N$ is related to the frequency $\omega$, or $\nu$, by

$$
N=\frac{\nu}{g^{2}} \int B^{2} \mathrm{~d}^{3} \rho .
$$

The energy of the system is

$$
E=\frac{\mu}{g^{2}} \int \mathscr{E} \mathrm{d}^{3} \rho,
$$

with the energy density $\mathscr{E}$ given by

$$
\mathscr{E}=\frac{1}{2}(\boldsymbol{\nabla} A)^{2}+\frac{1}{2}(\boldsymbol{\nabla} B)^{2}+\frac{1}{2}\left(\nu^{2}+\kappa^{2} A^{2}\right) B^{2}+\frac{1}{8}\left(A^{2}-1\right)^{2} .
$$

From (2.71) to (2.74), $\nu$ may be regarded as a function of $N$ and a functional of $B(\boldsymbol{\rho})$. Upon substituting $\nu=\nu(N, B)$ into (2.74), we may express $E$ as a function of $N$ and a functional of $A(\boldsymbol{\rho})$ and 
$B(\boldsymbol{\rho})$. Equations $(2.70)$ can also be derived by keeping $N$ fixed and setting the functional derivatives

$$
\delta E / \delta A(\boldsymbol{\rho})=\delta E / \delta B(\boldsymbol{\rho})=0
$$

The soliton solutions are analytical continuations of the plane wave solutions to $\omega<m$, i.e. $v<\kappa$.

The lowest-energy soliton solutions are spherically symmetric. The boundary conditions are

$$
\begin{aligned}
& \mathrm{d} A / \mathrm{d} \rho=\mathrm{d} B / \mathrm{d} \rho=0, \quad \text { at } \rho=0 . \\
& A=1, \quad B=0, \quad \text { at } \rho=x .
\end{aligned}
$$

Since (2.70) are second-order equations, with (2.76) there are still two more free parameters, which can be $A(0)$ and $B(0)$. For given $\nu$ and $\kappa$ with $\nu<\kappa$, adjusting $A(0)$ and $B(0)$ so that boundary conditions at infinity (2.77) are satisfied, one obtains a soliton solution. Figure 10 gives $E$ versus $N$ and $N$ versus $\omega$ for $\kappa=1$.

In this example, the lowest $\omega$ for the soliton solution is zero, as we shall prove. Consider a trial function in which $A=0$ and $B=B_{11} r^{-1} \sin \omega r$ inside a very large sphere of radius $R$, with

$$
\omega R=\pi
$$

Outside the sphere, $A=1-\mathrm{e}^{-(r-R) / l}$ with $l \ll R$ and $B=0$. Because of $(2.72)$ and $(2.78)$, the constant $B_{0}$ is $\propto N^{1 / 2}$. From (2.73) and (2.74), the corresponding energy is given by

$$
E=\pi N / R+\left(\pi \mu^{2} / 6 g^{2}\right) R^{3}+\mathrm{O}\left(R^{2}\right) .
$$

Setting $\partial E / \partial R=0$, we find, for large $N, R \propto N^{1: 4}$ and

$$
E \propto N^{3: 4} \ll m N .
$$

Since the correct lowest energy $E_{\min }$ at the same $N$ must be smaller than this trial function calculation, we derive, as $N \rightarrow x$.

$$
\omega \rightarrow E_{\min } / N \leq \mathrm{O}\left(N^{-1 / 4}\right) \rightarrow 0 .
$$

As $\omega$ varies from () to $m$, the $E$ (soliton) versus $N$ curve behaves in the same characteristic way as that shown in fig. 9.

One can show that on the lower branch of the $E$ versus $N$ curve in fig. 9 . for $N$ between $N_{c}$ and $x$ (the corresponding $\omega$ varies between $\omega_{c}>0$ and 0 ), the soliton solution is classically stable against small perturbations. As we shall discuss in the next section, quantum solitons along the same branch between $\mathrm{C}$ and $\mathrm{S}$ are only metastable, but their lifetimes could be quite long; those between $\mathrm{S}$ and $\propto$ are stable classically as well as quantum mechanically. Solitons on the upper branch in fig. 9 are not stable even classically. It is very common to have cusps on the $E$ versus $N$ curve for solitons in three spacedimensions. In section 3 we shall prove that whenever a cusp arises, a new mode of small perturbation acquires a zero frequency; thus on one side of the cusp the mode carries real frequency (stable vibration), but on the other side the frequency becomes complex, signifying instability.

Besides the examples discussed in this section, there are many nontopological soliton solutions that have been studied in the literature [22, 33-55]. 

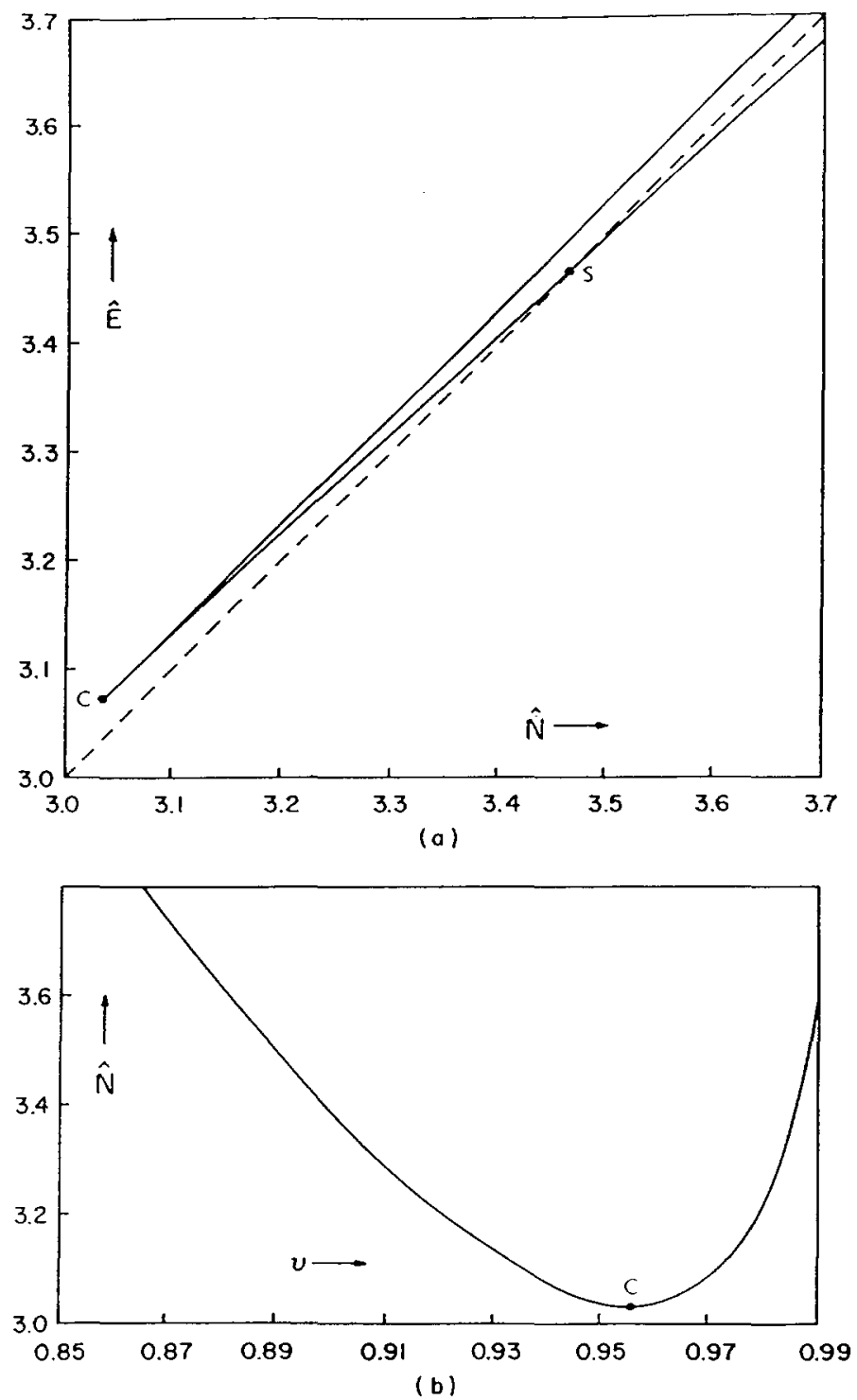

Fig. 10. $\hat{E}$ versus $\hat{N}$ and $\hat{N}$ versus $\nu$ curves for $\kappa=m / \mu=1$ in the neighborhood of the critical points, where $\hat{E}=(8 \pi m)^{-1} E g^{2}, \hat{N}=(8 \pi)^{-1} N g^{2}$, and $\nu=\omega / \mu$. The dashed line in (a) is $\hat{E}=\hat{N}$, or $E=N m$.

\section{Stability and quantization}

\subsection{General discussion}

In this section, we shall first examine the classical stability of nontopological soliton solutions. Let $\phi$ be a spherical symmetric equilibrium solution from the previous section, written in terms of a real $\sigma(r)$ function

$$
\phi=(1 / \sqrt{2}) \sigma(\boldsymbol{r}) \mathrm{e}^{-\mathrm{i} \omega t},
$$


and $\delta \phi$ be the small perturbation around $\phi$. For classical stability, one needs only to examine the characteristic frequency $v$ of the linearized perturbative equation in $\delta \phi$. Because of the invariance under time reversal, only $\nu^{2}$ appears. When all $\nu^{2}$ are real and greater than zero, solution $\phi$ is classically stable under small perturbation. For any complex $\nu^{2}$, or real $\nu^{2}<0, \delta \phi$ grows exponentially; for $\nu^{2}=0, \delta \phi$ increases linearly in $t$. There are two spurious $\nu^{2}=0$ solutions of $\delta \phi$ which are not related to instability (and should be excluded from the beginning). One is due to the phase invariance (2.2), and the other is related to the invariance under a space translation. By keeping the particle number $N$ fixed in the perturbation, we can eliminate the first spurious $\nu^{2}=0$ solution. The other one is removed by keeping the total momentum fixed.

As $\omega$ decreases from $m$ to its minimum value (for $D \geq 3$ ) nontopological solitons generally encounter one or more cusps on the $M$ versus $N$ curve. (See, e.g. figs. 9 and 10.) At the cusp, say $\omega=\omega_{c}$, one has

$$
\mathrm{d} N / \mathrm{d} \omega=0, \quad \mathrm{~d} M / \mathrm{d} \omega=0 .
$$

When $\omega$ changes infinitesimally from $\omega_{\mathrm{c}}-\varepsilon$ to $\omega_{\mathrm{c}}+\varepsilon$, one moves across a cusp along the $M$ versus $N$ curve. The variation $\delta \phi$ of the corresponding equilibrium solution can be readily seen to be $O(\varepsilon)$, whereas the variations $\delta N$ and $\delta M$ are both $\mathrm{O}\left(\varepsilon^{2}\right)$, because of eqs. (3.2). Consequently, $\delta \phi$ also satisfies the linearized perturbation equation with characteristic frequency $\nu=0$, and vice versa. This follows since $\delta N=\mathrm{O}\left(\varepsilon^{2}\right)$ is consistent with $N$ being constant in the perturbation, $\delta M=\mathrm{O}\left(\varepsilon^{2}\right)$ implies $\nu=0$, and the center of mass is obviously unchanged by $\delta \phi$ (since e.g., the entire $M$ versus $N$ curve in figs. 9 and 10 refers to solutions whose centers are at $r=0)$. Thus, the appearance of a cusp indicates the onset of $a$ new mode of instability $[17,56]$.

In section 3.2, we introduce a set of collective coordinates which separates the degrees of freedom associated with the phase and the space translations from other degrees of freedom. The resulting equation for the small perturbation $\delta \phi$ is free of the two spurious $\nu=0$ solutions. The canonical quantization procedure $[22,57,58]$ is discussed in section 3.3 ; as we shall see, it is closely related to the formulation of classical stability.

\subsection{Stability of the classical soliton solution}

The Lagrangian density is given by (2.43), with $D=3$. We introduce four collective coordinates, the three components $R_{k}(t)$ of the center of mass position vector $(k=1,2,3)$ and an over-all phase variable $\theta(t)$. Let us write

$$
\begin{aligned}
& \phi=(1 / \sqrt{2}) \mathrm{e}^{-\mathrm{i} \theta(t)}[\sigma(\boldsymbol{r}-\boldsymbol{R})+\chi(\boldsymbol{r}-\boldsymbol{R}, t)] . \\
& \chi(\boldsymbol{r}-\boldsymbol{R}, t) \equiv \chi_{\mathrm{R}}(\boldsymbol{r}-\boldsymbol{R}, t)+\mathrm{i} \chi_{\mathrm{I}}(\boldsymbol{r}-\boldsymbol{R}, t) .
\end{aligned}
$$

with $\chi_{\mathrm{R}}, \chi_{\mathrm{I}}$ and $\theta$ all real. Because $\phi$ is invariant under the infinitesimal variations $\theta \rightarrow \theta+\delta \theta$ and $\chi_{1} \rightarrow \chi_{1}+\sigma \delta \theta$, we impose the constraint

$$
\int \sigma \chi_{\mathrm{I}} \mathrm{d}^{3} r=0
$$

which removes the spurious $\nu=0$ perturbative solution connected with the phase invariance. To 
eliminate the three degrees of freedom related to the space translation, we further require

$$
\int \chi_{\mathrm{R}} \nabla \sigma \mathrm{d}^{3} r=0
$$

Expand

$$
\begin{aligned}
& \chi_{\mathrm{R}}(r, t)=\sum_{n=5}^{\infty} \operatorname{Re} q_{n}(t) f_{n}(r), \\
& \chi_{\mathrm{I}}(r, t)=\sum_{n=5}^{\infty} \operatorname{Im} q_{n}(t) f_{n}(r),
\end{aligned}
$$

where $\left\{f_{n}(r)\right\}$ with $n=1,2, \ldots, 5,6, \ldots$ denotes a complete set of orthonormal complex functions which satisfy

$$
\int f_{n}^{*} f_{n^{\prime}} \mathrm{d}^{3} r=\delta_{n n^{\prime}}
$$

with $f_{k} \propto \partial \sigma / \partial r_{k}(k=1,2,3)$ and $f_{4} \propto \sigma$. Otherwise the functions $f_{n}(n=5,6, \ldots)$ are arbitrary.

The collective coordinates $R, \theta$ and the vibrational coordinates $q_{n}$ can be put together as one single column matrix:

$$
q=\left(R_{1}, R_{2}, R_{3}, \theta, q_{5}, q_{6}, \ldots\right)^{\mathrm{T}}
$$

with the superscript $\mathrm{T}$ denoting the transpose of the corresponding row matrix. The Lagrangian $L=\int \mathscr{L} \mathrm{d}^{3} r$ can then be written as

$$
L=\frac{1}{2} \dot{q}^{\dagger} \mathcal{M} \dot{q}-\mathscr{V}(q)
$$

where $\dot{q}=\mathrm{d} q / \mathrm{d} t$, and

$$
\mathscr{V}(q)=\int \mathrm{d}^{3} r\left[\nabla \phi^{\dagger} \cdot \nabla \phi+U\left(\phi^{\dagger} \phi\right)\right]
$$

Substituting (3.3)-(3.7) in the Lagrangian density (2.43), we find

$$
\mathcal{M}_{k k^{\prime}}=\int \mathrm{d}^{3} r\left(\frac{1}{3}(\boldsymbol{\nabla} \sigma)^{2} \delta_{k k^{\prime}}+2 \frac{\partial \sigma}{\partial r_{k}} \frac{\partial \chi_{\mathrm{R}}}{\partial r_{k^{\prime}}}+\frac{\partial \chi_{\mathrm{R}}}{\partial r_{k}} \frac{\partial \chi_{\mathrm{R}}}{\partial r_{k^{\prime}}}+\frac{\partial \chi_{\mathrm{I}}}{\partial r_{k}} \frac{\partial \chi_{\mathrm{I}}}{\partial r_{k^{\prime}}}\right),
$$

where (and throughout the rest of this section), the subscripts

$$
k, k^{\prime}=1,2,3 \text {. }
$$

Denote from now on

$$
n, n^{\prime}=5,6, \ldots
$$

The other matrix elements are 


$$
\begin{aligned}
& M_{44}=\int \mathrm{d}^{3} r\left(\sigma^{2}+2 \sigma \chi_{\mathrm{R}}+\chi_{\mathrm{R}}^{2}+\chi_{\mathrm{I}}^{2}\right), \\
& M_{4 k}=M_{k 4}=\int \mathrm{d}^{3} r\left(-2 \frac{\partial \sigma}{\partial r_{k}} \chi_{\mathrm{I}}+\chi_{\mathrm{R}} \frac{\partial \chi_{\mathrm{I}}}{\partial r_{k}}-\chi_{\mathrm{I}} \frac{\partial \chi_{\mathrm{R}}}{\partial r_{k}}\right), \\
& M_{k n}=M_{n k}=-\frac{1}{2} \int \mathrm{d}^{3} r\left(\left(f_{n}+f_{n}^{*}\right) \frac{\partial \chi_{\mathrm{R}}}{\partial r_{k}}-\mathrm{i}\left(f_{n}-f_{n}^{*}\right) \frac{\partial \chi_{\mathrm{I}}}{\partial r_{k}}\right) . \\
& M_{4 n}=M_{n+4}=\frac{1}{2} \int \mathrm{d}^{3} r\left[\mathrm{i}\left(f_{n}-f_{n}^{*}\right) \chi_{\mathrm{R}}+\left(f_{n}+f_{n}^{*}\right) \chi_{\mathrm{I}}\right], \\
& M_{n n^{\prime}}=\delta_{n n^{\prime}}
\end{aligned}
$$

Regarding the Lagrangian as a function $L(q, \dot{q})$, the conjugate momentum of $q$ is $p=\partial L / \partial \dot{q}$, i.e.

$$
p=\mathscr{M} \dot{q} \equiv\left(P_{1}, P_{2}, P_{3}, N, p_{5}, p_{6}, \ldots\right)^{\top}
$$

where $p$ is a column matrix and the superscript $T$ denotes the transpose; the particle number is

$$
N=\partial L / \partial \dot{\theta}
$$

and the total momentum can be set to zero, i.e.

$$
P_{k}=\partial L / \partial \dot{R}_{k}=0
$$

In terms of these conjugate variables $q$ and $p$, the (classical) Hamiltonian becomes

$$
H=\frac{1}{2} p^{\dagger} \mathcal{M}^{-1} p+\eta(q)
$$

The advantage of the canonical transformation, changing our original variables $\phi$ and $\dot{\phi}$ to $q$ and $p$, lies in making explicit the conservation of both the particle number $N$ and the total momentum $\boldsymbol{P}$. Since both $\mathscr{V}(q)$ and the matrix $\mathcal{M},(3.12)$ and (3.13)-(3.20), are independent of $\theta$ and $\boldsymbol{R}$, we have

$$
\dot{N}=\mathrm{d} N / \mathrm{d} t=-\partial H / \partial \theta=0, \quad \dot{P}_{k}=\mathrm{d} P_{k} / \mathrm{d} t=-\dot{\partial} H / \partial R_{k}=0 .
$$

In the following discussion of the perturbation around the soliton, we shall regard $\chi_{\mathrm{R}}, \chi_{\mathrm{I}}$ and their conjugate momenta

$$
p_{\mathrm{R}}=\partial L / \partial \dot{\chi}_{\mathrm{R}}, \quad p_{\mathrm{I}}=\partial L / \partial \dot{\chi}_{\mathrm{I}}
$$

as $\mathrm{O}(\varepsilon)$, where $\varepsilon$ is an infinitesimal. Regarding $\sigma$ and $N$ as the zeroth order, we expand the Hamiltonian $H$ in powers of $\varepsilon$ :

$$
H=H_{0}+H_{1}+H_{2}+\cdots
$$


with $H_{n}=\mathrm{O}\left(\varepsilon^{n}\right)$. Likewise, define $\mathcal{M}_{0}$ to be the zeroth order $\mathcal{M}$-matrix. Write

$$
\mathcal{M}=M_{0}+\Delta
$$

where $\Delta$ contains $\mathrm{O}(\varepsilon)$ as well as higher orders. The inverse of $\mathcal{M}$ is

$$
\mathcal{M}^{-1}=\mathcal{M}_{0}^{-1}-\mathcal{M}_{0}^{-1} \Delta \mathcal{M}_{0}^{-1}+\mathcal{M}_{0}^{-1} \Delta \mathcal{M}_{0}^{-1} \Delta \mathcal{M}_{0}^{-1}+\cdots .
$$

From (3.13)-(3.20), we see

$$
\mathscr{M}_{0}=\left(\begin{array}{llllllll}
M_{0} & & & & & 0 & \\
& M_{0} & & & & & \\
& & M_{0} & & & & \\
& & & I & & & \\
& & & & 1 & & \\
0 & & & & & 1 & \\
& & & & & & \ddots
\end{array}\right) \text {, }
$$

where

$$
M_{0}=\frac{1}{3} \int \mathrm{d}^{3} r(\boldsymbol{\nabla} \sigma)^{2}, \quad I=\int \mathrm{d}^{3} r \sigma^{2} .
$$

In the expansion, $\sigma$ is regarded as a given function of $r$ that satisfies the time-independent equation of motion (2.47), with $\omega$ appearing as a parameter. (So far, logically $\omega$ is independent of $N$.) It is straightforward to verify that

$$
H_{0}=\frac{1}{2}\left(N^{2} I^{-1}+\omega^{2} I\right)+M_{0}, \quad H_{1}=\left(\omega^{2}-N^{2} I^{-2}\right) \int \mathrm{d}^{3} r \sigma \chi_{\mathrm{R}}
$$

Because $\omega$ and $N$ are independent parameters, we have the liberty to set

$$
N=I \omega
$$

In that case

$$
H_{0}=M_{0}+I \omega^{2}, \quad H_{1}=0 .
$$

In the next section we shall see that, for a sufficiently small nonlinear coupling $g$, the relation (3.33) ensures a power series expansion in $g$.

Setting $N=I \omega$, we find the second order Hamiltonian $H_{2}$ in (3.27) to be

$$
H_{2}=\mathscr{K}+\mathscr{V}_{\mathrm{R}}+\mathscr{V}_{\mathrm{I}}
$$

in which 


$$
\begin{aligned}
& \mathscr{H}=\frac{1}{2} \sum_{n}\left|p_{n}-\omega \mathcal{M}_{4 n}\right|^{2}, \\
& \mathscr{V}_{\mathrm{R}}=\frac{1}{2} \int \mathrm{d}^{3} r \chi_{\mathrm{R}} h_{\mathrm{R}} \chi_{\mathrm{R}}+\frac{2 \omega^{2}}{T^{2}}\left(\int \mathrm{d}^{3} r \chi_{\mathrm{R}} \sigma\right)^{2}, \\
& \mathscr{V}_{\mathrm{I}}=\frac{1}{2} \int \mathrm{d}^{3} r \chi_{\mathrm{I}} h_{\mathrm{I}} \chi_{\mathrm{I}}+\frac{2 \omega^{2}}{M_{0}^{2}}\left(\int \mathrm{d}^{3} r \chi_{\mathrm{I}} \nabla \sigma\right)^{2},
\end{aligned}
$$

where $h_{\mathrm{R}}$ and $h_{1}$ are defined by

$$
h_{\mathrm{R}}=-\boldsymbol{\nabla}^{2}+\frac{1}{2} \mathrm{~d}^{2} U / \mathrm{d} \sigma^{2}-\omega^{2}, \quad h_{\mathrm{l}}=-\boldsymbol{\nabla}^{2}+(1 / 2 \sigma) \mathrm{d} U / \mathrm{d} \sigma-\omega^{2} .
$$

Let $\psi_{\mathrm{R} i}$ and $\psi_{\mathrm{I} j}$ be the eigenstates of $h_{\mathrm{R}}$ and $h_{1}$ :

$$
\begin{aligned}
& h_{\mathrm{R}} \psi_{\mathrm{R} i}=\lambda_{\mathrm{R} i} \psi_{\mathrm{R} i}, \\
& h_{\mathrm{I}} \psi_{\mathrm{I} j}=\lambda_{\mathrm{I} j} \psi_{\mathrm{I} j} .
\end{aligned}
$$

Without any loss of generality we may choose all the $\psi_{\mathrm{R} i}$ to be real. For any given particle number $N$, we shall consider the lowest energy classical soliton solution. That is the lower branch in fig. 9. In accordance with $(2.47)$, we have

$$
h_{1} \sigma=0
$$

Because of translational invariance, $\sigma(\boldsymbol{r}+\boldsymbol{\varepsilon})$ must also satisfy the same formal equation (3.42). For an infinitesimal $\varepsilon$, its deviation from the original soliton solution $\sigma(r)$ is $\varepsilon \cdot \nabla \sigma(r)$ which leads to three p-state eigenfunctions of $h_{\mathrm{R}}$ all with zero eigenvalues, i.e. by differentiating (3.42),

$$
h_{\mathrm{R}} \psi_{k}=0 \text {, }
$$

where

$$
\psi_{k} \propto \partial \sigma / \partial r_{k} .
$$

Since the lowest s-state eigenvalue of $h_{\mathrm{R}}$ must be lower than the lowest p-state eigenvalue, $h_{\mathrm{R}}$ has at least one negative s-state eigenvalue (later we shall show that there is actually only one such negative eigenvalue). From (3.42), we see that $h_{1}$ has one zero eigenvalue s-state eigenfunction $\propto \sigma$; since $\sigma$ has no node, all other eigenvalues of $h_{\mathrm{I}}$ are positive definite (as can be derived by using the argument given in the paragraph following (2.53)).

Consider the eigenvalue equations generated by

$$
\delta \mathscr{V}_{\mathrm{R}} / \delta \chi_{\mathrm{R}}=\Lambda_{\mathrm{R}} \chi_{\mathrm{R}}, \quad \delta \mathscr{V}_{\mathrm{I}} / \delta \chi_{\mathrm{I}}=\Lambda_{\mathrm{I}} \chi_{\mathrm{I}}
$$

Labelling the respective c number eigenfunctions $\Psi_{\mathrm{R} i}$ and $\Psi_{\mathrm{I} j}$ and their associated eigenvalues $\Lambda_{\mathrm{R} i}$ and $\Lambda_{\mathrm{I} j}$, we have 


$$
h_{\mathrm{R}} \Psi_{\mathrm{R} i}+\frac{4 \omega^{2}}{I}\left(\int \mathrm{d}^{3} \sigma \Psi_{\mathrm{R} i}\right) \sigma=\Lambda_{\mathrm{R} i} \Psi_{\mathrm{R} i}
$$

and

$$
h_{\mathrm{I}} \Psi_{\mathrm{I} j}+\frac{4 \omega^{2}}{M_{0}}\left(\int \mathrm{d}^{3} \nabla \sigma \Psi_{\mathrm{l} j}\right) \cdot \nabla \sigma=\Lambda_{\mathrm{I} j} \Psi_{\mathrm{I} j}
$$

We may again choose these eigenfunctions $\Psi_{\mathrm{R} i}$ and $\Psi_{\mathrm{I} j}$ to be real.

Next, we shall establish the positivity of $\Lambda_{\mathrm{R} i}$ and $\Lambda_{\mathrm{I} j}$, i.e.

$$
\Lambda_{\mathrm{R} i} \geq 0, \quad \Lambda_{\mathrm{I} j} \geq 0 .
$$

Furthermore, there are three $\Lambda_{\mathrm{R} k}=0$ and one $\Lambda_{\mathrm{I} j}=0$ (the corresponding $\Psi_{\mathrm{R} k} \propto \nabla_{k} \sigma$ and $\Psi_{\mathrm{l} j} \propto \sigma$ ); all other $\Lambda_{\mathrm{R} i}$ and $\Lambda_{\mathrm{I} j}$ are positive definite.

Proof. Multiplying (3.47) by $\Psi_{\mathrm{I} j}$ and integrating over all space, we see that the positivity of $h_{\mathrm{I}}$ implies $\Lambda_{\mathrm{I} j} \geq 0$. Define the functional

$$
E(f) \equiv \int\left[\frac{1}{2} \omega^{2} f^{2}+\frac{1}{2}(\boldsymbol{\nabla} f)^{2}+U\left(\frac{1}{2} f^{2}\right)\right] \mathrm{d}^{3} r
$$

where $f(r)$ is a real function of $r$ and $\omega=\omega(f)$ is regarded as another functional of $f$ given by $\omega \int f^{2} \mathrm{~d}^{3} r=N=$ a fixed number. Take $N>N_{\mathrm{C}}$ (i.e. along the lower branch in fig. 9), so that the soliton solution $\sigma$ exists. Since $\sigma(r) \mathrm{e}^{-\mathrm{i} \omega t}$ is the lowest energy soliton solution for a given $N$, the minimum of $E(f)$ must be at $f=$ the same $\sigma$. Hence, any infinitesimal variation $f=\sigma+\varepsilon \Psi_{\mathrm{R} i}$ has to introduce a positive change $\varepsilon^{2} \Lambda_{\mathrm{R} i}$, which leads to $\Lambda_{\mathrm{R} i} \geq 0$, and proves the positivity (3.48). Next, we examine the number of zero eigenvalues among $\Lambda_{\mathrm{R} i}$ and $\Lambda_{\mathrm{I} j}$.

For $\psi_{\mathrm{I} j} \propto \sigma,(3.47)$ reduces to $(3.41)$; the corresponding $\Lambda_{\mathrm{I} j}=0$. All other $\Lambda_{\mathrm{I} j}$ are positive definite (since the same holds for $\lambda_{\mathrm{I} j}$ ). For $\Psi_{\mathrm{R} i} \propto \nabla_{i} \sigma$, the corresponding $\Lambda_{\mathrm{R} i}=0$, because (3.46) likewise reduces to (3.40). The spherical symmetry of $\sigma$ ensures that the same reduction occurs for other eigenfunctions, except when $\Psi_{\mathrm{R} i}$ is s-wave. Thus, for nonspherical eigenstates, there are only three $\mathrm{p}$-wave $\Lambda_{\mathrm{R} i}=0$; all other $\Lambda_{\mathrm{R} i}$ are positive definite (since the same holds for all other eigenvalues $\lambda_{\mathrm{R} i}$ of nonspherical solutions of $h_{\mathrm{R}}$ ). To establish the positive definiteness of all s-wave $\Lambda_{\mathrm{R} i}$, let $\psi_{1}, \psi_{2}, \ldots$ denote the set of all s-wave eigenfunctions of $h_{\mathrm{R}}$ :

$$
h_{\mathrm{R}} \psi_{i}=\lambda_{i} \psi_{i}
$$

The $\psi_{i}$ are chosen to be real and orthonormal, with

$$
\int \psi_{i} \psi_{j} \mathrm{~d}^{3} r=\delta_{i j}
$$

and their eigenvalues arranged so that

$$
\lambda_{1}<\lambda_{2}<\lambda_{3}<\cdots
$$


(For convenience, we may enclose the system in a large but finite sphere, so that, in accordance with the Sturm-Liouville theorem, these s-wave eigenvalues become discrete and non-degenerate.) As shown before, $\lambda_{1}<0$.

Similarly, let $\left\{\Psi_{i}\right\}$ be the set of all s-wave $\Psi_{\mathrm{R} i}$, and $\Lambda_{i}$ their corresponding eigenvalues $\Lambda_{\mathrm{R} i}$. The positivity of $\Lambda_{\mathrm{R} i}$ established before implies $\Lambda_{i} \geq 0$. It can be readily verified that by using (3.46) and expanding $\Psi_{i}$ in terms of the set $\left\{\psi_{j}\right\}$, the corresponding eigenvalue $z=\Lambda_{i}$ is the root of

$$
\operatorname{det}|\lambda-z+b \tilde{b}|=0
$$

where $\lambda$ is a diagonal matrix with $\lambda_{i}$ as its diagonal matrix elements, $b$ is a column matrix, whose components are

$$
b_{i}=\frac{2 \omega}{I^{1 / 2}} \int \sigma \psi_{i} \mathrm{~d}^{3} r
$$

and $\tilde{b}$ its transpose. Introducing

$$
a \equiv(\lambda-z)^{-1 / 2} b
$$

we have

$$
\lambda-z+b \tilde{b}=(\lambda-z)^{1 / 2}(1+a \tilde{a})(\lambda-z)^{1 / 2} .
$$

Hence, if $z \neq \lambda_{i}$ for any $\lambda_{i},(3.53)$ is satisfied if $\operatorname{det}|1+a \tilde{a}|$ is zero, i.e.

$$
\mathscr{G}(z) \equiv 1+\sum_{i} \frac{b_{i}^{2}}{\lambda_{i}-z}=0 .
$$

From (3.31) and (3.33), we have

$$
\delta N=I \delta \omega+2 \omega \int \sigma \delta \sigma \mathrm{d}^{3} r
$$

where, on account of (3.42),

$$
h_{\mathrm{R}} \delta \sigma-2 \omega \sigma \delta \omega=0
$$

Therefore, at $z=0, \mathscr{G}(z)$ is given by

$$
\mathscr{G}(0)=I^{-1} \mathrm{~d} N / \mathrm{d} \omega<0 .
$$

In accordance with (3.57), the derivative $\mathrm{d} \mathscr{G}(z) / \mathrm{d} z$ is always positive. When $z$ moves from $-\infty$ to $\lambda_{1}-$, $\mathscr{G}(z)$ increases from 1 to $+\infty$. Then $\mathscr{G}(z)$ jumps from $+\infty$ to $-x$ as $z$ crosses $\lambda_{1}$. When $z$ increases from $\lambda_{1}+$ to $\lambda_{2}-, \mathscr{G}(z)$ changes from $-\infty$ to $+\infty$. Because $\mathscr{G}(0)<0$, we have for the lowest eigenvalue $A_{1}$,

$$
0<\Lambda_{1}<\lambda_{2}
$$


Because $\lambda_{1}<0, \Lambda_{1}$ is also between $\lambda_{1}$ and $\lambda_{2}$. Likewise, each successive $\Lambda_{n}$ lies between $\lambda_{n}$ and $\lambda_{n+1}$ (which confirms the assertion made before (3.57), i.e. for $z$ being the root of (3.53), $z \neq \lambda_{i}$ for any $\lambda_{i}$ ). Thus, all $\Lambda_{i}$ are positive definite. This completes the proof of the statement following (3.48).

Construct two sets of functions,

$$
\left\{\Psi_{\mathrm{R} i}\right\}, \quad\left\{\Psi_{\mathrm{I} j}\right\}
$$

where the former contains all the eigenstates of (3.46) except the three $\Psi_{k} \propto \nabla_{k} \sigma$, and the latter contains all the eigenstates of (3.47) except $\sigma$. The expansions

$$
\chi_{\mathrm{R}}=\sum_{i} q_{\mathrm{R} i} \Psi_{\mathrm{R} i}, \quad \chi_{\mathrm{I}}=\sum_{j} q_{\mathrm{I} j} \Psi_{\mathrm{I} j}
$$

clearly satisfy the constraints (3.5) and (3.6). We can express (3.37) and (3.38) as

$$
\begin{aligned}
& \mathscr{V}_{\mathrm{R}}=\frac{1}{2} \sum_{i=1}^{\infty} \Lambda_{\mathrm{R} i} q_{\mathrm{R} i}^{2}, \\
& \mathscr{V}_{\mathrm{I}}=\frac{1}{2} \sum_{j=1}^{\infty} \Lambda_{\mathrm{I} j} q_{\mathrm{I} j}^{2}
\end{aligned}
$$

in which all $\Lambda_{\mathrm{R} i}$ and $\Lambda_{\mathrm{I} j}$ are $>0$. It is convenient to define

$$
p_{\mathrm{R}}=\left(\begin{array}{c}
p_{\mathrm{R} 1} \\
p_{\mathrm{R} 2} \\
\vdots
\end{array}\right), \quad p_{1}=\left(\begin{array}{c}
p_{11} \\
p_{12} \\
\vdots
\end{array}\right), \quad q_{\mathrm{R}}=\left(\begin{array}{c}
q_{\mathrm{R} 1} \\
q_{\mathrm{R} 2} \\
\vdots
\end{array}\right), \quad q_{1}=\left(\begin{array}{c}
q_{11} \\
q_{12} \\
\vdots
\end{array}\right)
$$

From (3.35)-(3.38) and (3.64), (3.65), we have

$$
H_{2}=\frac{1}{2}\left(\tilde{p}_{\mathrm{R}} p_{\mathrm{R}}+\tilde{p}_{\mathrm{I}} p_{\mathrm{I}}\right)+\frac{1}{2} \tilde{q}_{\mathrm{R}}\left(\Lambda_{\mathrm{R}}+\Gamma \tilde{\Gamma}\right) q_{\mathrm{R}}+\frac{1}{2} \tilde{q}_{\mathrm{I}}\left(\Lambda_{\mathrm{I}}+\tilde{\Gamma} \Gamma\right) q_{\mathrm{I}}+\tilde{p}_{\mathrm{R}} \Gamma q_{\mathrm{I}}-\tilde{p_{\mathrm{I}}} \tilde{\Gamma} q_{\mathrm{R}},
$$

where $\Lambda_{\mathrm{R}}$ and $\Lambda_{\mathrm{I}}$ are diagonal matrices whose diagonal matrix elements are $\Lambda_{\mathrm{R} i}$ and $\Lambda_{\mathrm{I} j}$ and the matrix elements of $\Gamma$ are

$$
\Gamma_{i j}=-\omega \int \mathrm{d}^{3} r \Psi_{\mathrm{R} i} \Psi_{\mathrm{I} j}
$$

We may cast the above expression (3.66) into a more compact form by introducing

$$
\eta \equiv\left(\begin{array}{l}
\mathscr{P} \\
2
\end{array}\right)
$$

where

$$
\mathscr{P} \equiv\left(\begin{array}{c}
p_{\mathrm{R}} \\
p_{\mathrm{I}}
\end{array}\right), \quad \mathscr{Q} \equiv\left(\begin{array}{c}
q_{\mathrm{R}} \\
q_{\mathrm{I}}
\end{array}\right) .
$$


Equation (3.66) for $H_{2}$ can then be written

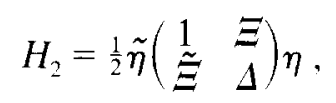

where

$$
\Xi=-\tilde{\Xi}=\left(\begin{array}{cc}
0 & \Gamma \\
-\tilde{\Gamma} & 0
\end{array}\right), \quad \Delta=\left(\begin{array}{cc}
\Lambda_{\mathrm{R}}+\Gamma \tilde{\Gamma} & 0 \\
0 & \Lambda_{1}+\Gamma \tilde{\Gamma}
\end{array}\right)
$$

The Hamilton equation for $H_{2}$ is

$$
\frac{\partial \eta}{\partial t}=\left(\begin{array}{cc}
\Xi & -\Delta \\
1 & \Xi
\end{array}\right) \eta
$$

Examining its normal mode solution $\eta=\eta_{N}$, where

$$
\eta_{N}(t) \propto \mathrm{e}^{-\mathrm{i} \Omega \Omega_{N} t}
$$

we find

$$
\left(\begin{array}{cc}
\Xi-\mathrm{i} \Omega_{N} & -\Delta \\
1 & \Xi+\mathrm{i} \Omega_{N}
\end{array}\right) \eta_{N}=0
$$

Thus, $\Omega_{N}$ is the root of the quadratic equation

$$
-c_{1}+c_{2} \Omega_{N}+\Omega_{N}^{2}=0
$$

where

$$
c_{1}=\mathscr{2}_{N}^{+}\left(\begin{array}{cc}
A_{\mathrm{R}} & 0 \\
0 & \Lambda_{\mathrm{I}}
\end{array}\right) \mathscr{Q}_{N}, \quad c_{2}=\mathscr{2}_{N}^{+}\left(\begin{array}{cc}
0 & -2 \mathrm{i} \Gamma \\
2 \mathrm{i} \tilde{\Gamma} & 0
\end{array}\right) \mathscr{Q}_{N}
$$

and $\mathscr{Q}_{N}$ is the coordinate column vector part of $\eta_{N}$, chosen to satisfy the normalization condition

$$
\mathscr{Q}_{N}^{\div} \mathscr{Q}_{N}=1
$$

The solution of $(3.75)$ is

$$
\Omega_{N}=\frac{1}{2}\left[-c_{2} \pm\left(c_{2}^{2}+4 c_{1}\right)^{1 / 2}\right]
$$

Since $c_{1}$ is real and positive, and $c_{2}$ is real, hence

$$
\text { all } \Omega_{N} \text { are real }
$$

Thus the entire lower branch in fig. 9 is classically stable. From the argument given in section 3.1 , we conclude that the upper branch solutions are all unstable. 


\subsection{Quantization}

From the classical Hamiltonian (3.24), by following the standard canonical procedure, we have the quantum operator

$$
H=\frac{1}{2} \mathscr{F}^{-1} p^{\dagger} \mathcal{M}^{-1} \mathscr{F} p+\mathscr{V}(q)
$$

where the components of $p$, given by (3.21), now denote the differential operators

$$
P_{k}=-\mathrm{i} \partial / \partial R_{k}, \quad N=-\mathrm{i} \partial / \partial \theta, \quad p_{n}=-\mathrm{i} \partial / \partial q_{n},
$$

and $\mathscr{I}(q)$ is the Jacobian given by

$$
\mathscr{J}(q)=\sqrt{\operatorname{det} \mathscr{M}(q)}
$$

Since $\mathscr{I}(q), \mathcal{M}(q)$ and $\mathscr{V}(q)$ are all independent of the collective coordinates $\boldsymbol{R}$ and $\theta$, we have

$$
[\boldsymbol{P}, H]=0,[N, H]=0 .
$$

The Schrödinger equation for the state vector $\mid>$ can be written as

$$
H|\rangle=E|\rangle \text {. }
$$

Let $g$ be the nonlinear coupling. Following the analysis (2.33)-(2.35) of section 2, we see that in the expansion (3.27) $H_{0}$ is proportional to $g^{-2}$ and $H_{1}$ is proportional to $g^{-1}$. For $g$ sufficiently small, in order for $H_{0}$ to give the correct energy to $\mathrm{O}\left(g^{-2}\right)$, we must have $H_{1}|\rangle=0$. (Otherwise, $q_{n}$ would be $\mathrm{O}\left(g^{-1}\right)$ which induces an $\mathrm{O}\left(g^{-2}\right)$ energy from $\left.H_{1}+H_{2}+\cdots\right)$ Thus, we require

$$
N|\rangle=I \omega|\rangle \text {. }
$$

Because $\theta$ is cyclic with a period of $2 \pi$, the eigenvalue of its conjugate momentum $N$ must be an integer. Hence only the classical solution with $I \omega$ being integer can be quantized.

We may expand the energy in a power series in $g^{2}$,

$$
E=E_{0}+E_{2}+E_{4}+\cdots,
$$

where $E_{0} \sim \mathrm{O}\left(g^{-2}\right), E_{2} \sim \mathrm{O}\left(g^{2} E_{0}\right) \sim \mathrm{O}\left(g^{0}\right), E_{4} \sim \mathrm{O}\left(g^{2}\right)$, etc. From the previous section, we can write $\mathrm{H}_{2}$, given by (3.66), in terms of positive eigenvalues $\Omega_{N}$,

$$
H_{2}=\sum_{N}\left(\frac{1}{2}+\mathcal{N}_{N}\right) \Omega_{N}+E_{\text {vac }},
$$

where $E_{\mathrm{vac}}$ is the vacuum energy without the soliton and $\mathcal{N}_{N}$ is the occupation number of the $N$ th normal mode. By treating the higher order $H_{n}$ (with $n>2$ ) as perturbation, we derive a systematic expansion for the quantum solution. 
The canonical quantization procedure in this section follows closely that of Christ and Lee [22]. Similar methods have been used in quantizing topological and nontopological soliton solutions $[57,58,59]$. The quantum soliton solutions can also be obtained through various forms of functional integration techniques [60-65], the Green's function approaches $[23,66]$, and the variational methods [67-69]. Some of these techniques will be described in section 5 .

\section{Bose liquid}

\subsection{General discussion}

In this section, we give a description of Bose liquid, based on nontopological soliton solutions. Much of the fundamental approach is already contained in the well-known work of London [70], Landau [71] and many others on liquid He. Nevertheless, as will be discussed in sections 4.2 and 4.3 , the basic theorem on the formation of the Bose liquid is relatively recent.

In the usual treatment of Bose-Einstein $(\mathrm{BE})$ condensation, one begins with an ideal boson system as the zeroth approximation; the interaction is treated as a perturbation. Since solitons are bound-state solutions, such a perturbation series naturally misses their existence. In this chapter, we represent the boson system by a nonrelativistic nonlinear quantized local field $\phi$, and use the lowest-energy c number solution $\phi=\sigma$ (instead of free particles) as the zeroth-order approximation. In this way, the nonlinearity of the problem is recognized at the outset. In the case of solitons, as discussed in the previous sections, the interaction must be partially attractive, and $\sigma$ is typically $\mathrm{O}\left(g^{-1}\right)$ with $g$ the appropriately defined nonlinear coupling. A systematic perturbation series in $g^{2}$ can be developed. By having the new series start with $\mathrm{O}\left(\mathrm{g}^{-2}\right)$. one takes into account the soliton solution, which forms the Bose liquid.

The same method is also applicable when the interaction is purely repulsive. (In that case, the condensate is a gas, not a liquid.) As will be shown in section 4.4, when applied to a dilute system of nonrelativistic Bose hard spheres of number density $\rho$, diameter $a$ and mass $m$ in three dimensions $(D=3)$, it gives the well-known ground state energy per particle $[72,73](\hbar=1)$,

$$
E_{\mathrm{gd}} / N=2 \pi(a \rho / m)\left[1+\frac{128}{15}\left(\rho a^{3} / \pi\right)^{1 / 2}+\mathrm{O}\left(\rho a^{3} \ln a\right)\right]
$$

as well as the excited level formula.

The characterization of a Bose liquid is interesting, since it possesses both the liquid nature as well as the usual BE phase coherence. In section 4.5, we discuss the spontaneous symmetry breaking mechanism. Our original Hamiltonian has two symmetries: the space-translational invariance and a $\mathrm{U}(1)$ phase invariance. The breaking of the former gives the "soliton" condensation in the liquid-gas transition (as that between liquid HeI and the helium gas) and of the latter the BE transition (as the $\Lambda$-transition between $\mathrm{HeI}$ and $\mathrm{HeII}$ ). At very low temperatures, both can be broken. A precise criterion for these two phase transitions can be obtained. Since the stability of the nontopological soliton depends on the phase invariance, its symmetry breaking has to be handled with care. As we shall see. this subtlety is, in turn, connected with the essential difference between the canonical and grand canonical averages. 


\subsection{Nonrelativistic solitons $[30,74,75]$}

Consider a nonrelativistic local (zero-spin) field theory in which the boson operator $\phi(x, t)$ satisfies the commutation relation

$$
\left[\phi(x, t), \phi^{\dagger}\left(x^{\prime}, t\right)\right]=\delta^{D}\left(x-x^{\prime}\right)
$$

where, as before, a dagger denotes the hermitian conjugate, $\boldsymbol{x}$ is the $D$-dimensional space-vector, and $t$ the time. The Hamiltonian $H(\phi)$ and the particle number $N(\phi)$ are given by (in the unit $\hbar=1$ )

$$
\begin{aligned}
& H=\int\left(\frac{1}{2 m} \nabla \phi^{\dagger} \cdot \nabla \phi+U\left(\phi^{\dagger} \phi\right)\right) \mathrm{d}^{D} x, \\
& N=\int \phi^{\dagger} \phi \mathrm{d}^{D} x,
\end{aligned}
$$

where $m$ is the boson mass.

As in the relativistic theory, because $H$ is invariant under the phase transformation

$$
\phi \rightarrow \mathrm{e}^{-\mathrm{i} \theta} \phi
$$

$N$ is conserved. For stability, $U$ is bounded from below; in addition,

$$
U \rightarrow \infty \text { when }|\phi| \rightarrow \infty
$$

as would be the case for any matter at an infinite density (without gravity). For physical applications, $\phi$ would be a phenomenological boson field representing a composite of an even number of fermions (in the case of helium, $\phi$ represents the He atom); hence, there is

an ultraviolet cutoff $\Lambda$

to ensure the finiteness of the theory for any $U$. We may take $U$ to be a polynomial of $\phi^{\dagger} \phi$, with

$$
U \rightarrow f \times\left(\phi^{\dagger} \phi\right)^{2}, \text { when }|\phi| \rightarrow 0 \text {. }
$$

(A quadratic term, constant $\times \phi^{\dagger} \phi$, in $U$ gives an energy linear in $N$, which can always be dropped.) The theorem proved in section 2.2 can be readily extended to the nonrelativistic case:

If there exists a range in $|\phi|$ however small, in which $U$ is attractive (i.e. $U<0$ ) however weakly, then in any space-dimension $D$ when $N$ is sufficiently large, the ground state is a soliton (i.e. a bound state or a Bose liquid) with a fixed density $\rho_{\mathrm{s}}$ and a nonzero binding energy

$$
\Delta>0
$$

per particle. 
The nonrelativistic version differs from the relativistic case in some technical aspects, e.g., $N$ depends on $\dot{\phi}$ in a relativistic theory, but not for a nonrelativistic system. For clarity, a proof is given below. We discuss in this section only the c number part; the quantum theory will be treated in the next section.

By minimizing $H$ at a fixed $N$ with respect to $\phi$ and labeling the resulting (lowest energy) solution

$$
\phi \equiv \sigma(x)
$$

we find that $\sigma$ satisfies

$$
-(1 / 2 m) \boldsymbol{\nabla}^{2} \sigma+\frac{1}{2} \mathrm{~d} U / \mathrm{d} \sigma=\nu \sigma
$$

where $\nu$ is the Lagrangian multiplier and $\sigma$ can be chosen to be real. This equation is of the same form as (2.47), with only the differences that (i) as $\sigma \rightarrow 0, U \rightarrow f \sigma \sigma^{4}$ here, whereas $U \rightarrow m^{2} \sigma^{2}$ in the relativistic case (in accordance with (2.48)), and (ii) an attraction means $U<0$ here; but in a relativistic case, $U<m^{2} \sigma^{2}$.

In our case, let $-\sigma^{2} \Delta$ be the parabola tangent to $U$ at $\sigma=\sigma_{s}$, as illustrated in fig. 11 for (i) $f<0$ and (ii) $f>0$. By following the same argument given in section 2.2 , we see that in any space-dimension $D$, when $N$ is sufficiently large, a soliton solution exists, with $\nu$ negative but greater than $-\Delta$. As $N \rightarrow x$, $\nu \rightarrow-\Delta$ and the soliton energy $E(N)$ becomes

$$
E=-N \Delta+\mathrm{O}\left(N^{(D-1) / D}\right)
$$

as in (2.57). This establishes (4.8). Because (4.10) is generated by $\delta[H(\sigma)-\nu N(\sigma)]=0$, we have

$$
\mathrm{d} E / \mathrm{d} N=\nu .
$$

Before leaving the $\mathrm{c}$ number theory, it is useful to have some of the expressions for perturbations around the soliton solution. Write

$$
\phi=\mathrm{e}^{-\mathrm{i} \theta}\left[\sigma\left(x-x_{0}\right)+\chi_{\mathrm{R}}+\mathrm{i} \chi_{\mathrm{I}}\right]
$$

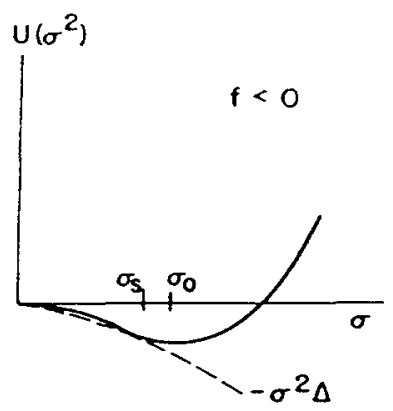

(i)

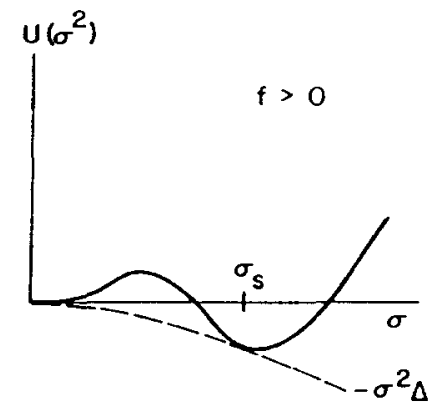

(ii)

Fig. 11. $U\left(\sigma^{2}\right)$ is the self-interaction energy density of the boson field $\sigma$. As $\sigma \rightarrow 0, U \rightarrow f \sigma^{+}$. The assumption that $U$ is partially attractive means $U^{\prime}$ must be negative in some region of $\sigma$. (i) gives an example of $f<0$ and (ii) is for $f>0$. The parabola $-\sigma^{2} \Delta$ is tangent to $U$ at $\sigma=\sigma$. 
where $\theta$ and $x_{0}$ are constants, $\chi_{\mathrm{R}}$ and $\chi_{\mathrm{I}}$ are real functions which will be regarded as being small, $\mathrm{O}(\varepsilon)$. Because $N(\phi)$ and the total momentum

$$
\boldsymbol{P}(\phi) \equiv-\mathrm{i} \int \phi^{\dagger} \boldsymbol{\nabla} \phi \mathrm{d}^{D} x
$$

are conserved, we may impose the constraints

$$
\begin{aligned}
& N(\phi)=N(\sigma)=\int \sigma^{2} \mathrm{~d}^{D} x, \\
& \boldsymbol{P}(\phi)=\boldsymbol{P}(\sigma)=0 .
\end{aligned}
$$

Consequently,

$$
\begin{aligned}
& \int \chi_{\mathrm{R}} \sigma \mathrm{d}^{D} x=-\frac{1}{2} \int\left(\chi_{\mathrm{R}}^{2}+\chi_{\mathrm{I}}^{2}\right) \mathrm{d}^{D} x=\mathrm{O}\left(\varepsilon^{2}\right) \\
& \int \chi_{\mathrm{I}} \nabla \sigma \mathrm{d}^{D} x=\frac{1}{2} \int\left(\chi_{\mathrm{R}} \nabla \chi_{\mathrm{I}}-\chi_{\mathrm{I}} \nabla \chi_{\mathrm{R}}\right) \mathrm{d}^{D} x=\mathrm{O}\left(\varepsilon^{2}\right) .
\end{aligned}
$$

By using (4.10) and (4.17), we find the Hamiltonian (4.2) to be given by

$$
H(\phi)=E(N)+\int\left(\chi_{\mathrm{R}} h_{\mathrm{R}} \chi_{\mathrm{R}}+\chi_{\mathrm{I}} h_{\mathrm{I}} \chi_{\mathrm{I}}\right) \mathrm{d}^{D} x+\mathrm{O}\left(\varepsilon^{3}\right)
$$

where $E(N)=H(\sigma)$ is the zeroth-order energy

$$
\begin{aligned}
& h_{\mathrm{R}}=-(1 / 2 m) \nabla^{2}+\frac{1}{2} \mathrm{~d}^{2} U / \mathrm{d} \sigma^{2}-\nu, \\
& h_{\mathrm{l}}=-(1 / 2 m) \nabla^{2}+(1 / 2 \sigma) \mathrm{d} U / \mathrm{d} \sigma-\nu .
\end{aligned}
$$

Hence,

$$
\begin{aligned}
& h_{\mathrm{R}} \nabla \sigma=0, \\
& h_{\mathrm{I}} \sigma=0 .
\end{aligned}
$$

As in section 3.2 , we can readily establish the following properties:

(1) From (4.22), we see that $h_{\mathrm{R}}$ has $D$ p-wave eigenfunctions $\propto \partial \sigma / \partial x_{n}$ (where $n=1,2, \ldots, D$ ) all with zero eigenvalues. Consequently, $h_{\mathrm{R}}$ has one s-wave eigenfunction with a negative eigenvalue. All other eigenvalues of $h_{\mathrm{R}}$ are positive definite.

(2) From (4.23), it follows that $h_{\mathrm{I}}$ has one s-wave eigenfunction $\propto \sigma$ with a zero eigenvalue; all its other eigenvalues are positive definite.

(3) Because $\phi=\sigma$ gives the lowest $H(\phi)$ at a fixed $N(\phi)$, we have, from (4.15), (4.17) and (4.19), if $\int \chi_{\mathrm{R}} \sigma \mathrm{d}^{D} x=0$ 


$$
\int \chi_{\mathrm{R}} h_{\mathrm{R}} \chi_{\mathrm{R}} \mathrm{d}^{I)} x \geq 0
$$

Likewise, for any $\chi_{1}$

$$
\int \chi_{1} h_{1} \chi_{1} \mathrm{~d}^{b} x \geq 0
$$

The equality signs occur only when $\chi_{\mathrm{R}}$ is $x_{\partial} \partial / \partial x_{n}$ and $\chi_{1} x_{\sigma} \sigma$ (which are in turn connected to the translational invariance $x_{0} \rightarrow x_{0}+$ constant and the phase invariance $\theta \rightarrow \theta+$ constant of the theory). Hence, by requiring

$$
\int \chi_{\mathrm{R}} \sigma \mathrm{d}^{\prime \prime} x=\int \chi_{\mathrm{R}} \nabla \sigma \mathrm{d}^{\prime \prime} x=0
$$

we derive

$$
\int \chi_{\mathrm{R}} h_{\mathrm{R}} \chi_{\mathrm{R}} \mathrm{d}^{\prime \prime} x>0
$$

Likewise,

$$
\int \chi_{\mathrm{I}} h_{1} \chi_{\mathrm{I}} \mathrm{d}^{D} x>0 \text { if } \int \chi_{\mathrm{I}} \sigma \mathrm{d}^{D} x=0
$$

(4) As in (2.33)-(2.35), the $\varepsilon$-expansion in (4.13) and (4.17)-(4.19) can also be expressed in an alternative way. Define an overall nonlinear coupling $g$ by requiring, for $\hat{\sigma} \equiv g \sigma$,

$$
U(\sigma)=g^{-2} u(\hat{\sigma})
$$

with $u(\hat{\sigma})$ not explicitly dependent on $g$. Thus, e.g., the coupling $f$ in (4.7) can be written as

$$
f= \pm g^{2}
$$

Equation (4.10) becomes

$$
-(1 / 2 m) \Gamma^{2} \hat{\sigma}+\frac{1}{2} \mathrm{~d} u / \mathrm{d} \hat{\sigma}=\nu \hat{\sigma}
$$

which is $g$-independent. Hence, $\hat{\sigma}=\mathrm{O}(1), \sigma=\mathrm{O}\left(g^{\prime}\right)$ and $E(N)$ and $N(\sigma)$ are both $\mathrm{O}\left(g^{2}\right)$. Regard $\chi_{\mathrm{R}}$ and $\chi_{\mathrm{I}}$ as $\mathrm{O}(1)$. The expansion (4.19) can be viewed as an expansion in $g$, with $\mathrm{O}\left(\varepsilon^{3}\right)$ replaced by $\mathrm{O}(\mathrm{g})$.

\subsection{Quantum theory}

Write (4.13) as

$$
\phi=\mathrm{e}^{-\mathrm{i} \theta}\left[\sigma\left(\boldsymbol{x}-\boldsymbol{x}_{01}\right)+\chi\right]
$$


where

$$
\chi=\chi_{\mathrm{R}}+\mathrm{i} \chi_{\mathrm{I}}
$$

and $\theta, \boldsymbol{x}_{0}$ are constants, as before. In quantum theory, $\chi_{\mathrm{R}}$ and $\chi_{1}$ are Hermitian operators. On account of (4.1), the commutator between $\chi(x, t)$ and $\chi^{\dagger}\left(\boldsymbol{x}^{\prime}, t\right)$ is $\delta^{D}\left(\boldsymbol{x}-\boldsymbol{x}^{\prime}\right)$. We have

$$
\begin{aligned}
& {\left[\chi_{\mathrm{I}}(x, t), \chi_{\mathrm{R}}\left(x^{\prime}, t\right)\right]=-\frac{1}{2} \mathrm{i} \delta^{D}\left(x-x^{\prime}\right),} \\
& {\left[\chi_{\mathrm{R}}(x, t), \chi_{\mathrm{R}}\left(x^{\prime}, t\right)\right]=\left[\chi_{\mathrm{I}}(x, t), \chi_{\mathrm{I}}\left(x^{\prime}, t\right)\right]=0 .}
\end{aligned}
$$

The operators $\boldsymbol{P}(\psi)$ and $N(\psi)$, defined by (4.14) and (4.3) are both conserved (i.e. commute with $H$ ). Let $\boldsymbol{P}(\sigma)=0$ and $N(\sigma)$ be the corresponding c number, given by $\int \sigma^{2} \mathrm{~d}^{D} x$. Equations (4.15) and (4.16) become conditions on the state vectors:

$$
N(\phi)|\rangle=N(\sigma)|\rangle, \quad \boldsymbol{P}(\phi)|\rangle=0 .
$$

As in (4.29)-(4.31), we regard $\sigma=\mathrm{O}\left(g^{-1}\right)$ and $\chi=\mathrm{O}(1)$. To $\mathrm{O}\left(g^{-1}\right),(4.35)$ gives

$$
\int \chi_{\mathrm{R}} \sigma \mathrm{d}^{D} x|\rangle=\int \chi_{\mathrm{I}} \nabla \sigma \mathrm{d}^{D} x|\rangle=0
$$

Likewise, to $\mathrm{O}(1),(4.19)$ becomes the expansion

$$
H(\phi)|\rangle=\left(E(N)+\int\left(\chi_{\mathrm{R}} h_{\mathrm{R}} \chi_{\mathrm{R}}+\chi_{\mathrm{I}} h_{\mathrm{I}} \chi_{\mathrm{I}}\right) \mathrm{d}^{D} x+\mathrm{O}(g)\right)|\rangle
$$

where $E(N)=H(\sigma)$ is $\mathrm{O}\left(g^{-2}\right)$.

Because of (4.34), we may regard $\sqrt{2} \chi_{\mathrm{R}}$ as the generalized coordinate and $\sqrt{2} \chi_{\mathrm{I}}$ as its conjugate momentum. (This is a typical nonrelativistic relation, quite different from the relativistic case.) Let $\left\{f_{i}(\boldsymbol{x})\right\}$ be a set of real complete orthonormal functions, with

$$
f_{0} \propto \sigma, \quad f_{1} \propto \partial \sigma / \partial x_{1}, \ldots, \quad f_{D} \propto \partial \sigma / \partial x_{\mathrm{D}}
$$

otherwise, $f_{D+1}, f_{D+2}, \ldots$ can be arbitrary. Expand

$$
\sqrt{2} \chi_{\mathrm{R}}=\sum_{\alpha=0}^{D} q_{\alpha} f_{\alpha}+\sum_{n=1}^{\infty} Q_{n} f_{D+n}, \quad \sqrt{2} \chi_{\mathrm{I}}=\sum_{\alpha=0}^{D} p_{\alpha} f_{\alpha}+\sum_{n=1}^{\infty} \Pi_{n} f_{D+n} .
$$

From (4.36) and (4.38), we have, to $O(1)$,

$$
q_{0}|\rangle=0, \quad p_{\beta}|\rangle=0, \quad \text { where } \beta=1,2, \ldots, D .
$$

In the representation that $p_{0}, q_{1}, \ldots, q_{D}$ and $Q_{n}$ are diagonal, we may set $q_{0}=\mathrm{i} \partial / \partial p_{0}, p_{\beta}=-\mathrm{i} \partial / \partial q_{\beta}$ and $\Pi_{n}=-\mathrm{i} \partial / \partial Q_{n}$. Equation (4.40) implies that the state vector should be independent of $p_{0}$ and $q_{\beta}$. 
Therefore, it is only a function of $Q_{1}, Q_{2}, \ldots$ Hence, the Schrödinger wave function can be written as

$$
\langle Q \mid\rangle, \text { where } Q \equiv\left(\begin{array}{c}
Q_{1} \\
Q_{2} \\
\vdots
\end{array}\right)
$$

Let

$$
\Pi \equiv-\mathrm{i}\left(\begin{array}{c}
\partial / \partial Q_{1} \\
\partial / \partial Q_{2} \\
\vdots
\end{array}\right)
$$

By using $(4.22),(4.23)$ and $(4.40)$, we can rewrite $(4.37)$ in the $Q$-representation. The corresponding time-dependent Schrödinger equation

$$
H(\phi)|\rangle=-\mathrm{i}(\partial / \partial t)|\rangle
$$

becomes

$$
\bar{H}(Q, \Pi)\langle Q \mid\rangle=-\mathrm{i}(\partial / \partial t)\langle Q \mid\rangle
$$

where

$$
\bar{H}(Q, \Pi)=E(N)+H_{2}(Q, I)+\mathrm{O}(g), \quad H_{2}(Q, I I)=\frac{1}{2} \tilde{Q} A Q+\frac{1}{2} \tilde{I} B I I
$$

with the tilde denoting the transpose as before, and the matrix elements of $A$ and $B$ being given by

$$
A_{i j}=\int f_{D+i} h_{\mathrm{R}} f_{D+j} \mathrm{~d}^{(D} x, \quad B_{i j}=\int f_{D+i} h_{1} f_{D+j} \mathrm{~d}^{\prime \prime} x
$$

and $i, j=1,2,3, \ldots$ In terms of

$$
\mathscr{R} \equiv \sum_{n=1}^{\infty} Q_{n} f_{D+n} . \quad \mathscr{I} \equiv \sum_{n=1}^{\infty} I_{n} f_{l, n},
$$

$H_{2}$ becomes

$$
H_{2}=\frac{1}{2} \int\left(\mathscr{R} h_{\mathrm{R}} \mathscr{R}+\mathscr{I} h_{\mathrm{I}} \mathscr{I}\right) \mathrm{d}^{D} x
$$

Since by construction

$$
\int \mathscr{R} \sigma \mathrm{d}^{D} x=\int \mathscr{R} \boldsymbol{\nabla} \sigma \mathrm{d}^{D} x=0, \int \mathscr{I} \sigma \mathrm{d}^{(D} x=\int \mathscr{I} \boldsymbol{\nabla} \sigma \mathrm{d}^{D} x=0
$$

it follows from (4.26)-(4.28) that $H_{2}>0$ for any $\mathscr{R}$ and $\mathscr{I}$. Hence, the matrices $A$ and $B$ are both positive definite, besides being also real and symmetric. 
We may switch from the Schrödinger picture to the Heisenberg picture by regarding the operators $Q$ and $\Pi$ as $t$-dependent and the state vector $t$-independent. From (4.44) and (4.45), we find, neglecting $\mathrm{O}(\mathrm{g})$,

$$
\dot{Q}=B \Pi, \quad \dot{\Pi}=-A Q
$$

Define

$$
\bar{Q}=B^{-1 / 2} Q, \quad \bar{\Pi}=A^{-1 / 2} \Pi
$$

Hence,

$$
\ddot{\bar{Q}}=-B^{1 / 2} A B^{1 / 2} \bar{Q}, \quad \ddot{\bar{\Pi}}=-A^{1 / 2} B A^{1 / 2} \bar{\Pi} .
$$

The positive definiteness of $A$ and $B$ ensures that all eigenvalues of $A^{1 / 2} B A^{1 / 2}$ (which are the same as those of $B^{1 / 2} A B^{1 / 2}$ )

$$
\omega_{1}^{2}, \omega_{2}^{2}, \ldots>0
$$

A systematic expansion of $g$ is then possible.

To $\mathrm{O}\left(g^{-2}\right)$, the ground state energy is given by $E(N)$, the classical soliton expression. When $g$ is small, the character of the ground state remains determined by the zeroth-order term in the expansion. Hence, the theorem (see (4.8)) stated in the previous section holds in the quantum theory, provided that the $g$-expansion is valid.

As an example, consider the special case when the lowest-energy soliton solution $\sigma$ fills the entire volume $\Omega$, with $\sigma=\sigma_{\mathrm{s}}, N(\sigma)=\sigma_{\mathrm{s}}^{2} \Omega$ and

$$
E(N)=-N \Delta
$$

When $\sigma=\sigma_{\mathrm{s}}$ and $\nu=-\Delta$, we have, as shown in fig. 11, (i) and (ii),

$$
\nu \sigma^{2}-U\left(\sigma^{2}\right)=0
$$

Because $\nabla \sigma=0,(4.39)$ can be written as

$$
\sqrt{2} \chi_{\mathrm{R}}=\frac{1}{\sqrt{\Omega}}\left(q_{0}+\sum_{k \neq 0} Q_{k} \mathrm{e}^{\mathrm{i} k \cdot x}\right), \quad \sqrt{2} \chi_{\mathrm{I}}=\frac{1}{\sqrt{\Omega}}\left(p_{0}+\sum_{k \neq 0} \Pi_{k} \mathrm{e}^{-\mathrm{i} \boldsymbol{k} \cdot \boldsymbol{x}}\right),
$$

with

$$
Q_{k}=Q_{-k}^{\dagger}, \quad \Pi_{k}=\Pi_{-k}^{\dagger} .
$$

The corresponding $\mathrm{H}_{2}$ is

$$
H_{2}=\frac{1}{2} \sum_{k \neq 0}\left[\left(k^{2} / 2 m+2 m v_{\mathrm{s}}^{2}\right) Q_{k}^{\dagger} Q_{k}+\left(k^{2} / 2 m\right) \Pi_{k}^{\dagger} \Pi_{k}\right]
$$


where $v_{s}$ is the sound velocity given by

$$
v_{\mathrm{s}}=\left[(1 / 2 m)\left(\Delta+\frac{1}{2} \mathrm{~d}^{2} U / \mathrm{d} \sigma^{2}\right)\right]^{1: 2} \text { at } \sigma=\sigma_{\mathrm{s}}
$$

The frequency (4.52) is

$$
\omega_{k}=\left[k^{2} v_{s}^{2}+\left(k^{2} / 2 m\right)^{2}\right]^{1 / 2}
$$

Correspondingly, the spectrum for the low-lying excitations is

$$
\bar{H}=-N \Delta+\sum_{k} n_{k} \omega_{k}+\delta E
$$

where $n_{k}=0,1,2, \ldots$ and $\delta E$ denotes the shift of the zero-point energy. (An illustration of how to calculate $\delta E$ will be given in the next section.)

When $N \equiv \sigma^{2} \Omega>\sigma_{s}^{2} N$, then $\sigma>\sigma_{s}$; its value is determined by

$$
\nu=(1 / 2 \sigma) \mathrm{d} U / \mathrm{d} \sigma
$$

in accordance with (4.12) and

$$
E=U(\sigma) \Omega
$$

The number density $\rho$ is $\sigma^{2}$ and the pressure $p$ is

$$
p=-(\partial E / \partial \Omega)_{N}=-U+\frac{1}{2} \sigma \mathrm{d} U / \mathrm{d} \sigma
$$

The compressibility is

$$
\frac{1}{m} \frac{\mathrm{d} p}{\mathrm{~d} \rho}=\frac{1}{2 m}\left(-\frac{1}{2 \sigma} \frac{\mathrm{d} U}{\mathrm{~d} \sigma}+\frac{1}{2} \frac{\mathrm{d}^{2} U}{\mathrm{~d} \sigma^{2}}\right)=\frac{\sigma^{2}}{m} \frac{\mathrm{d}^{2} U}{\mathrm{~d}\left(\sigma^{2}\right)^{2}} .
$$

At $\sigma=\sigma_{\mathrm{s}}$ and $\nu=-\Delta$, by using (4.54) and (4.58), we find, as expected,

$$
v_{\mathrm{s}}=\left(m^{-1} \mathrm{~d} p / \mathrm{d} \rho\right)^{1 / 2} \text { at } \rho=\rho_{\mathrm{s}} .
$$

The same frequency spectrum (4.59) also holds for $\sigma>\sigma_{s}$, provided $v_{\mathrm{s}}$ is replaced by $v=[\mathrm{d} p / \mathrm{d}(m \rho)]^{1,2}$ at $\rho=\sigma^{2}$.

\subsection{Bose hard spheres}

In the local field-theoretic approximation, a Bose system of hard spheres of diameter $a$ and mass $m$ (in $D=3$ ) can be represented by

$$
U=g^{2}\left(\phi^{\dagger} \phi\right)^{2}, \text { with } g^{2}=2 \pi a / m
$$


In this case, there is no soliton solution. At a given $N$, the lowest-energy c number solution is $\sigma=$ constant in the entire volume $\Omega$, determined by (4.61):

$$
\nu=(1 / 2 \sigma) \mathrm{d} U / \mathrm{d} \sigma
$$

Hence, for the repulsive case

$$
\nu=2 g^{2} \rho>0, \text { where } \rho=\sigma^{2}=N / \Omega .
$$

The classical energy is

$$
E=g^{2} \sigma^{4} \Omega=2 \pi N \rho a / m
$$

By going through the same steps as from (4.55) to (4.65), but with $v_{\mathrm{s}}$ and $\sigma_{\mathrm{s}}$ replaced by $v$ and $\sigma$, we find the spectrum

$$
\bar{H}=E+\sum_{k \neq 0} n_{k} \omega_{k}+\delta E
$$

where $n_{k}=0,1,2, \ldots$, and

$$
\omega_{k}=\left[k^{2} v^{2}+\left(k^{2} / 2 m\right)^{2}\right]^{1 / 2}
$$

The parameter $v$ is the sound velocity determined by

$$
v^{2}=(1 / 2 m)\left(-v+\frac{1}{2} \mathrm{~d}^{2} U / \mathrm{d} \sigma^{2}\right)=4 \pi a \rho / m^{2},
$$

and $\delta E$ is the shift in the zero-point energy, which is the same as the sum of all one-loop diagrams in the presence of an external field $\left(\phi^{\dagger} \phi\right)_{\mathrm{ext}}=\sigma^{2}$,

$$
\delta E=\frac{1}{2} \sum_{k}\left[k^{2} v^{2}+\left(k^{2} / 2 m\right)^{2}\right]^{1 / 2}-\frac{1}{2} \sum_{k} k^{2} / 2 m+c_{(\mathrm{i})}+c_{(\mathrm{ii})},
$$

where $c_{(i)}$ and $c_{(i i)}$ are counter terms due to the renormalizations of the inertia (giving a subtraction term $\propto\left(\phi^{\dagger} \phi\right)_{\text {ext }}$ ) and the coupling constant $g^{2}$ (giving a subtraction term $\propto\left(\phi^{\dagger} \phi\right)_{\text {ext }}^{2}$ ) in a "renormalizable" $g^{2}\left(\phi^{\dagger} \phi\right)^{2}$ field theory, as illustrated by the diagrams (i) and (ii) in fig. 12 .

Since $c_{(\mathrm{i})}$ is $\mathrm{O}\left(g^{2}\right)=\mathrm{O}(a)$ and $c_{(\mathrm{ii})}$ is $\mathrm{O}\left(g^{4}\right)=\mathrm{O}\left(a^{2}\right)$, the inclusion of the counter terms $c_{(\mathrm{i})}$ and $c_{(\mathrm{ii})}$ in (4.73) is identical to subtracting the $\mathrm{O}\left(g^{2}\right)$ and $\mathrm{O}\left(g^{4}\right)$ terms in the one-loop summation (regarding $\rho$

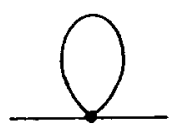

(i)

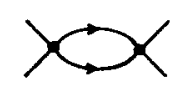

(ii)

Fig. 12. Diagrams for (i) the $\mathrm{O}\left(g^{2}\right)$ mass renormalization and (ii) the $\mathrm{O}\left(\mathrm{g}^{4}\right)$ coupling-constant renormalization of Bose hard spheres. 
as $g$-independent). We may formally expand $\left[k^{2} v^{2}+\left(k^{2} / 2 m\right)^{2}\right]^{1 / 2}$ as a power series in $v^{2}=\mathrm{O}\left(g^{2}\right)$, and simply drop its first three terms; this procedure corresponds exactly to the inclusion of the last three terms in (4.73). The result is

$$
\delta E=\frac{1}{2} \sum_{k}\left\{\left[k^{2} v^{2}+\left(k^{2} / 2 m\right)^{2}\right]^{1 / 2}-k^{2} / 2 m-m v^{2}+m^{3} v^{4} / k^{2}\right\} .
$$

As the volume $\Omega \rightarrow \infty, \Sigma_{k}=(2 \pi)^{-3} \Omega \int \mathrm{d}^{3} k$, and

$$
\delta E=\left(8 N m^{4} v^{5} / 15 \pi^{2}\right) \rho
$$

Thus, we rederive the well-known result [72,73] for the low-lying spectrum of a dilute system of Bose hard spheres:

$$
\bar{H}=2 \pi N \rho a m^{-1}\left[1+\frac{128}{15}\left(\rho a^{3} / \pi\right)^{1 / 2}\right]+\sum_{k} n_{k}(k / 2 m)\left(16 \pi a \rho+k^{2}\right)^{1 / 2}
$$

Using this level formula, one can calculate the partition function near zero temperature, $T=0$. For $T>0$ (but less than the critical temperature), higher excitations have to be included; those will be discussed in (4.100)-(4.105) below.

One may also verify directly that $c_{(\mathrm{i})}$ and $c_{(i \mathrm{i})}$ are indeed the last two terms in the sum (4.74). Recall that the nonrelativistic boson propagator is

$$
D\left(k_{0}, \boldsymbol{k}\right)=\mathrm{i}\left[k_{0}-\left(\boldsymbol{k}^{2} / 2 m\right)+\mathrm{i} \varepsilon\right]^{-1}, \quad \text { where } \varepsilon=0+.
$$

In fig. 12(i) the external line is $\sigma$; hence, its contribution to $\delta E$ is

$$
c_{(\mathrm{i})}=-\Omega g^{2}\left(\phi^{\dagger} \phi\right)_{\mathrm{ext}} \int D\left(k_{0}, k\right) \frac{\mathrm{d}^{4} k}{(2 \pi)^{4}}=-\sigma^{2} g^{2} \Omega \int \frac{\mathrm{d}^{3} k}{(2 \pi)^{3}} .
$$

Since $\sigma^{2} g^{2}=\frac{1}{2} m v^{2}$,

$$
c_{(\mathrm{i})}=-\sum_{k} \frac{1}{2} m v^{2}
$$

Likewise to $\mathrm{O}\left(g^{4}\right), \delta g^{2} \equiv(\text { unrenormalized coupling })^{2}-g^{2}$ at the zero-momentum transfer is

$$
\delta g^{2}=-2(-\mathrm{i}) g^{4} \int \frac{\mathrm{d}^{3} k}{(2 \pi)^{4}} \int \mathrm{d} k_{0} D\left(k_{0}, \boldsymbol{k}\right) D\left(-k_{0},-\boldsymbol{k}\right)=2 m g^{4} \int \frac{\mathrm{d}^{3} k}{(2 \pi)^{3} k^{2}}
$$

Note that $2 m g^{4} \sigma^{4}=8 \pi^{2} a^{2} \rho^{2} / m=\frac{1}{2} m^{3} v^{4}$. We obtain from fig. 12(ii),

$$
c_{(\mathrm{ii})}=\Omega\left(\delta g^{2}\right)\left(\phi^{\dagger} \phi\right)_{\mathrm{ext}}^{2}=\frac{1}{2} \sum_{k} \frac{m^{3} v^{4}}{k^{2}} .
$$

Equations (4.77) and (4.78) confirm the validity of (4.74). 


\subsection{Spontaneous symmetry breaking}

Return to the case when the interaction $U\left(\phi^{\dagger} \phi\right)$ is partially attractive. The ground state (in a very large volume $\Omega$ and for sufficiently large $N$ ) is a soliton; at very low temperature $T$, the system is a Bose liquid. The Hamiltonian $H$ has two symmetries: the translational invariance and the phase symmetry (4.4). The purpose of this section is to show that the symmetry breaking of the translational invariance is connected with the liquid-gas transition, whereas the breaking of the phase symmetry is related to the Bose-Einstein condensation. As we shall see, in order to give a precise formulation, it is convenient to adopt the canonical ensemble average for the former, but the grand canonical ensemble average for the latter.

The underlying physical reason for this difference is that in the canonical ensemble the total particle number $N$ is fixed: in a two-phase gas-liquid region, if the average density $N / \Omega$ is less than the liquid density, then the lowest energy state consists of a gas-liquid separation which breaks the translational symmetry. On the other hand, $N$ is not fixed in the grand canonical ensemble: because of the surface energy associated with the gas-liquid separation, in an average over $N$, at the critical Gibbs chemical potential $\mu=\mu_{\mathrm{c}}$ for the phase transition, the average density $N / \Omega$ jumps suddenly from the gas density (at $\mu=\mu_{\mathrm{c}}-$ ) to the liquid density $\left(\mu=\mu_{\mathrm{c}}+\right.$ ), bypassing the gas-liquid separated configuration and without a spontaneous translational symmetry breaking. Similarly, since $N$ is conjugate to the overall phase-variable $\theta$, it is difficult to have a phase-symmetry breaking in the canonical ensemble. In order to describe the $\mathrm{BE}$ transition as a spontaneous breaking in the $\theta$-invariance, it is more convenient to use the grand canonical ensemble. The details are given below.

Let $H_{\mathrm{op}}, N_{\mathrm{op}}$ and $\boldsymbol{P}_{\mathrm{op}}$ be the quantum operators of the same forms given by (4.2), (4.3) and (4.14). Define

$$
\mathscr{H} \equiv-\mu N_{\mathrm{op}}+H_{\mathrm{op}},
$$

where $\mu$ is the Gibbs thermodynamical energy per particle. Enclose the system in a large, but finite, cube of volume $\Omega$ with periodic boundary conditions. Denote the average of any operator $O$ in the canonical ensemble by angle brackets,

$$
\langle O\rangle \equiv Q^{-1} \operatorname{trace}\left(\mathrm{e}^{-\beta H} O\right)
$$

where $\beta=\left(k_{\mathrm{B}} T\right)^{-1}$ with $k_{\mathrm{B}}$ the Boltzmann constant, and $Q$ is the partition function

$$
Q \equiv \text { trace } \mathrm{e}^{-\beta H}
$$

with the trace taken over all state vectors |\rangle satisfying $N_{\mathrm{op}}|\rangle=N|\rangle$. Denote the corresponding average in the grand canonical ensemble by square brackets,

$$
[O]=\mathscr{2}^{-1} \operatorname{Trace}\left(\mathrm{e}^{-\beta \mathscr{H}} O\right), \text { where } 2=\operatorname{Trace}^{-\beta \mathscr{H}}
$$

and the (capitalized) Trace is taken over the entire Hilbert space consisting of states of all possible $N$. Because $H$ and $\mathscr{H}$ are invariant under the phase transformation $\phi \rightarrow \phi \mathrm{e}^{-\mathrm{i} \theta}$, we have

$$
\langle\phi\rangle=[\phi]=0
$$


Both $H$ and $\mathscr{H}$ are also invariant under the space-translation $\phi(x) \rightarrow \phi(x+c)$, with $c$ arbitrary for an infinite volume (but parallel to the edges of the cube for a finite $\Omega$ ). Hence, in the infinite volume limit. keeping the average number density $\rho$ fixed,

$$
\left\langle\phi^{\dagger} \phi\right\rangle=\left[\phi^{\dagger} \phi\right]=\text { constant }=\rho \text {. }
$$

\subsubsection{Translational symmetry breaking}

In order to show the liquid gas difference in a Bose system, we may replace $H$ and $\mathscr{t}$ by

$$
\begin{aligned}
& H_{\xi}=H+\int \xi\left(x-x_{0}\right) \phi^{\dagger}(x) \phi(x) \mathrm{d}^{D} x, \\
& \mathscr{H}_{\xi}=\mathscr{H}+\int \xi\left(x-\boldsymbol{x}_{0}\right) \phi^{\dagger}(\boldsymbol{x}) \phi(x) \mathrm{d}^{D} x,
\end{aligned}
$$

where $\xi\left(x-x_{0}\right)$ is a small negative bounded c number function of $x$ with a finite range and having a minimum at $\boldsymbol{x}_{0}$. For a system of ideal bosons, or bosons with only repulsive interaction $U$, there are only different gas phases. Taking first the limit $\Omega \rightarrow \infty$ at a fixed average number density $\rho$, and then the limit $\xi \rightarrow 0$, we derive again (4.84), which can also be obtained by taking the limit $\xi \rightarrow 0$ first and then $\Omega \rightarrow \infty$.

For bosons whose interaction $U$ is partially attractive, at sufficiently low temperature $T$ the system is a Bose liquid, in accordance with our basic theorem. (However, the gas-liquid critical temperature $T_{\mathrm{c}}=0$ if $D=1$.) Consider first the canonical ensemble with $H$ replaced by $H_{\xi}$. Take $T=0$ and $\rho \equiv N / \Omega<\sigma_{s}^{2}$. Since the Bose liquid has a density $\sigma_{\mathrm{s}}^{2}, N<\sigma_{\mathrm{s}}^{2} \Omega$ means that the liquid does not fill the entire volume $\Omega$. (We assume the validity of the $g$-expansion, so that the $\sigma$-term in (4.32) dominates.) Keeping $\rho$ fixed and letting $\Omega \rightarrow \infty$ (the volume occupied by the liquid also approaches infinity, with its center of mass at $\boldsymbol{x}_{0}$ ), arrange $\boldsymbol{x}_{0} \rightarrow \infty$ in such a way that a part of the liquid surface remains at a finite position. Hence, in contrast to (4.84), when the overall average density $\rho$ is less than the liquid density. we have

$$
\lim _{\xi \rightarrow 0} \lim _{\Omega \rightarrow x}\left\langle\phi^{\dagger} \phi\right\rangle \neq \text { constant }
$$

This double limit can be converted into a single one if we set

$$
\int \xi \mathrm{d}^{D} x=\mathrm{O}\left(\Omega^{-\alpha}\right), \text { with } 0<\alpha<(D+2) / D
$$

so that $\int \xi \mathrm{d}^{D} x \rightarrow 0$ as $\Omega \rightarrow \infty$, and yet the infrared degeneracy is broken (i.e. the macro-liquid would still be centered at $\boldsymbol{x}_{0}$, since without $\xi$ the first excited state of such a macro-liquid moving as a whole in a volume $\Omega=L^{D}$ has a kinetic energy $\left.\sim L^{-2} N^{-1} \propto \Omega^{-(D+2) / D}\right)$. As we shall see, the same limit of the grand canonical average $\left[\phi^{\dagger} \phi\right]$ behaves quite differently.

Let $E_{\Omega}(N)$ be the lowest-energy solution in the c number theory when $\xi=0$ and $\Omega$ is large but finite (with $E_{x}(N)=E(N)$ given by (4.11)). The general shape of $E_{\Omega}(N)$ is illustrated in fig. 13. Let $U(\sigma)$ be of the form given by fig. 11(i); $-U$ has one maximum at $\sigma=\sigma_{0}$. It can be readily verified that the solution of $(4.10)$, for $\nu=0$, is a constant $\sigma=\sigma_{0}$ throughout the entire volume $\Omega$. Since $\partial E_{\Omega}(N) /$ $\partial N=\nu=0$, this corresponds to the minimum $M$ of $E_{\Omega}(N)$ with $N_{M}=\Omega \sigma_{1}^{2}$. As $\nu$ increases from zero, 


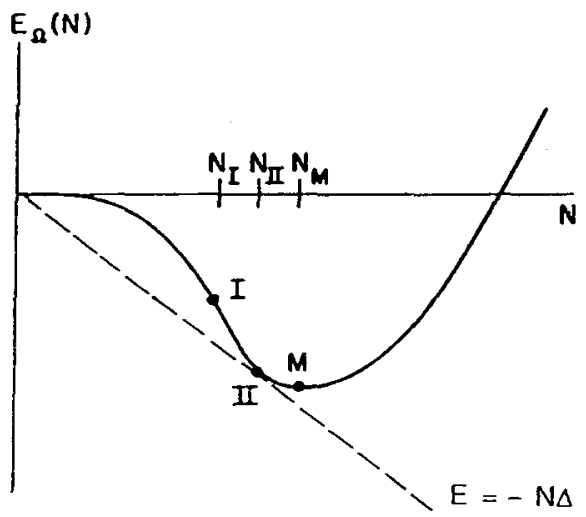

Fig. 13. The total energy $E_{\Omega}(N)$ of a Bose liquid versus $N$ (at a given volume $\Omega$ ). The point $I$ is the point of inflection. The dashed line $E=-N \Delta$ is tangent to $E_{0}(N)$ at II.

let $\sigma=\sigma_{\nu}$ denote the point when $u(\sigma) \equiv \nu \sigma^{2}-U$ reaches its maximum, i.e. $[\partial u(\sigma) / \partial \sigma]_{\nu}=0$ at $\sigma=\sigma_{\nu}$. The solution of (4.10) remains a constant $\sigma$, given by $\sigma=\sigma_{\nu}$, with the corresponding $N=\Omega \sigma_{\nu}^{2}>N_{M}$.

When $\nu$ is negative, a more complicated pattern of solutions emerges. Consider first $\nu=0-; u(\sigma)$ becomes negative for $\sigma$ near 0 . There are now two solutions of (4.10), both satisfying the periodic boundary conditions: one is a constant solution $\sigma=\sigma_{0}$ - (at the maximum of $u$ ), and the other is a soliton solution oscillating near $\sigma=0$. On the $E_{\Omega}(N)$ curve, the former is represented by a point at $N=N_{M}-$, but the latter by a point at $N=0+$. At both points, their slopes $\partial E_{\Omega}(N) / \partial N=\nu=0-$ are the same. As $\nu$ decreases from zero, on the $E_{\Omega}(N)$ curve, these two solutions move towards each other. We note that the corresponding curve $u(\sigma)$ now has two maxima, at $\sigma=0$ and $\sigma=\sigma_{\nu}$; in between there is a minimum at, say, $\sigma=\sigma_{v}^{\prime}$. The constant solution is $\sigma=\sigma_{v}$ and the other, soliton-like solution (since $\Omega$ is finite), oscillates around $\sigma_{v}^{\prime}$ between the two maxima. Decrease $\nu$ steadily until the maximum $\sigma_{\nu}$ coalesces with $\sigma_{\nu}^{\prime}$ at $\nu=\nu_{\mathrm{I}}<0$; the curve $u(\sigma)$ now has a point of inflection at $\sigma=\sigma_{\nu}=\sigma_{\nu}^{\prime}$. There is then only one single solution. Because $\partial E_{\Omega}(N) / \partial N=\nu$, this solution is represented by $N=N_{\mathrm{I}}$ which is also the point of inflection I of the curve $E_{\Omega}(N)$, shown in fig. 13.

The soliton-like solution has a surface energy, and it determines $E_{\Omega}(N)$ only between $N=0$ and $N_{\mathrm{I}}$. Beyond $N_{1}$, the lowest-energy solution is $\sigma=$ constant (which has no surface energy), determined by $\mathrm{d} u / \mathrm{d} \sigma=0$. In fig. 13, we draw the dashed straight line $E=-N \Delta$ tangent to $E_{\Omega}(N)$ at the point II. Because $N_{\mathrm{II}}>N_{\mathrm{I}}$, the corresponding lowest-energy solution is a constant $\sigma \equiv \sigma_{\mathrm{s}}$, and therefore $N_{\mathrm{II}}=\sigma_{\mathrm{s}}^{2} \Omega$.

Next, consider the grand partition function (still with $\Omega$ finite, $T=0, \xi=0$ and assuming the dominance of the $\mathrm{c}$ number solution). The Gibbs thermodynamical energy $\mu$ is determined by requiring

$$
\left[N_{\mathrm{op}}\right]=N=\rho \Omega \geq 0 .
$$

By construction, the function

$$
\mathscr{E}_{\Omega}(N) \equiv-\mu N+E_{\Omega}(N)
$$

when $\mu=-\Delta$ has two degenerate minima: $\xi_{\Omega}\left(N_{\mathrm{II}}\right)=\xi_{\Omega}(0)$. Hence $\xi_{\Omega}(N)$ has its minimum at $N=0$ if $\mu<-\Delta$, but at $N \geq N_{\text {II }}$ if $\mu \geq-\Delta$. Therefore, at $T=0,\left[N_{\text {op }}\right]$ versus $\mu$ has the same behavior, i.e. 
$\left[N_{\mathrm{op}}\right]=0$ if $\mu<-\Delta$ but jumps to $N \geq N_{11}$ if $\mu \geq-\Delta$. (Note that the gas density is zero at $T=0$.) Since the surface energy of the soliton solution is $\mathrm{O}\left(\Omega^{(D-1) / D}\right)$, the same conclusion holds after we introduce a small symmetry-breaking term $\xi$. (The $\xi$-dependent energy remains finite as $\Omega \rightarrow x$, and it becomes zero as $\xi \rightarrow 0$.) Thus, in the grand canonical ensemble the boundstate nature of the macroscopic solitons does not play any essential role. The grand canonical average of $\phi \phi$ is always a constant, just as in the purely repulsive case; its two-phase region consists of the probability distribution of the two pure phases, each filling the entire volume uniformly. In contrast, in accordance with (4.87), the canonical average of the Bose liquid can be quite different: in the two-phase region, because of the spontaneous symmetry breaking of the translational invariance, at $T=0$ the system consists of a single macroscopic soliton which occupies only part of the volume, leaving the remaining part vacant. In the infinite volume limit, both ensembles give the same intensive thermodynamic functions. All the above considerations can, of course, be generalized to $T \neq 0$. (For $D=1$, the gas-liquid critical temperature is 0.)

\subsection{2. $U(1)$ symmetry breaking}

In order to break the phase symmetry $\phi \rightarrow \phi \mathrm{e}^{-\mathrm{i} t}$, it is best to use the grand canonical ensemble, as we shall see. Consider the three-dimensional case, $D=3$. The operator $\phi$ satisfies the commutation relation

$$
\left[\phi(x, t), \phi^{\dagger}\left(x^{\prime}, t\right)\right]=\delta^{3}\left(x-x^{\prime}\right)
$$

In order to have a spontaneous symmetry breaking in the phase symmetry, we write

$$
\phi(x, t)=\sigma_{0}+\chi, \quad \chi=\sum_{k} a_{k} \mathrm{e}^{i k \cdot x} / \sqrt{\Omega},
$$

where $\sigma_{0}$ is a constant, $a_{k}$ and its hermitian conjugate $a_{k}^{\dagger}$ are annihilation and creation operators, with

$$
\left[a_{k}, a_{k^{\prime}}^{\dagger}\right]=\delta_{k k^{\prime}}
$$

As in (4.79), let

$$
\begin{aligned}
& \mathscr{H} \equiv H_{\mathrm{op}}-\mu N_{\mathrm{op}} . \\
& V\left(\phi^{\dagger} \phi\right) \equiv U\left(\phi^{\dagger} \phi\right)-\mu \phi^{\dagger} \phi,
\end{aligned}
$$

where $U$ is the interaction that appears in $H_{\mathrm{op}}$.

Expand $V\left(\phi^{\dagger} \phi\right)$ around $\phi^{\dagger} \phi=\left|\sigma_{0}\right|^{2}$ :

$$
V\left(\phi^{\dagger} \phi\right)=V_{0}+V_{0}^{\prime}\left(\phi^{\dagger} \phi-\left|\sigma_{0}\right|^{2}\right)+\frac{1}{2} V_{0}^{\prime \prime}\left(\phi^{\dagger} \phi-\left|\sigma_{0}\right|^{2}\right)^{2}+\cdots
$$

where $V_{0}=V\left(\left|\sigma_{0}\right|^{2}\right), \quad V_{0}^{\prime}=\mathrm{d} V\left(\left|\sigma_{0}\right|^{2}\right) / \mathrm{d}\left|\sigma_{0}\right|^{2}$ and $V_{0}^{\prime \prime}=\mathrm{d}^{2} V\left(\left|\sigma_{0}\right|^{2}\right) /\left(\mathrm{d}\left|\sigma_{0}\right|^{2}\right)^{2}$. At a given $\mu,\left|\sigma_{0}\right|^{2}$ is determined by requiring $V$ to be a minimum at $\phi^{+} \phi=\left|\sigma_{0}\right|^{2}$. Hence, $V_{0}^{\prime}=0$, i.e. 


$$
\begin{aligned}
& \mu=\mathrm{d} U\left(\left|\sigma_{0}\right|^{2}\right) / \mathrm{d}\left(\left|\sigma_{0}\right|^{2}\right), \\
& V_{0}^{\prime \prime} \equiv m v^{2} /\left|\sigma_{0}\right|^{2}>0,
\end{aligned}
$$

which also defines the parameter $v$.

So far, the minimum of $V$ only determines the magnitude of $\left|\sigma_{0}\right|^{2}$. Introduce an infinitesimal constant $j$ and define

$$
\mathscr{H}_{j} \equiv \mathscr{H}+\int\left(j \phi^{\dagger}+j^{*} \phi\right) \mathrm{d}^{D} x
$$

This makes the minimum of $V$ occur at a definite $\sigma_{0}$ whose phase $=\pi+$ phase of $j$; thereby, the symmetry breaking is achieved. For $j$ real and $\rightarrow 0, \sigma_{0}$ is real and $\mathscr{H}_{j} \rightarrow \mathscr{H}$. Neglecting the cubic $\left(\phi^{\dagger} \phi-\sigma_{0}^{2}\right)^{3}$ and higher order terms, we have (in normal products and retaining only up to the quadratic part of $a_{k}$ and $a_{k}^{\dagger}$ )

$$
\mathscr{H} \equiv V_{0}+\sum_{k}\left[\left(k^{2} / 2 m+2 m v^{2}\right) a_{k}^{\dagger} a_{k}+m v^{2}\left(a_{k} a_{-k}+a_{-k}^{\dagger} a_{k}^{\dagger}\right)\right]
$$

whose eigenvalues are

$$
\mathscr{E}=V_{0}+\sum_{k} n_{k}\left[k^{2} v^{2}+\left(k^{4} / 4 m^{2}\right)\right]^{1 / 2},
$$

where $n_{k}$ are positive integers, all $\mathrm{O}(1)$, related to $N$ (the expectation value of $N_{\mathrm{op}}$ ) by

$$
\sum_{k} n_{k}+\sigma_{0}^{2} \Omega=N
$$

(In (4.92), the sum over $a_{k}$ includes $k=0$; to determine $\sigma_{0}$ to the accuracy $\Omega^{-1 / 2}$, we require the grand canonical ensemble average $\left[a_{k=0}\right]=0$.) From the excitation spectrum, we see that the parameter $v$ is the sound velocity.

Let

$$
\sigma_{0}^{2}=y N / \Omega
$$

where $y$ is the fraction of the total number of particles that are in the zero-momentum state. The parameter $y$ characterizes the long-range order of the Bose condensate. Its temperature dependence can be determined by

$$
\Omega^{-1} \int \mathrm{d}^{3} x[\phi(x)]=(y \rho)^{1 / 2} .
$$

For the Bose hard spheres at a low but non-zero temperature, by using (4.66) and (4.97), we find the chemical potential to be

$$
\mu=2 g^{2} \sigma_{0}^{2}=4 \pi a y \rho / m
$$


and the sound velocity $v$ (at $0 \leq T<T_{c}$ ) given by

$$
v^{2}=2 g^{2} \sigma_{0}^{2} / m=4 \pi a y \rho / m^{2}
$$

in agreement with the well-known results [76]. [When $T=0$, we have $y=1 ;(4.105)$ reduces to (4.72).]

In general, for an interacting boson system, it is clear that, because the condensate density $\left|\sigma_{0}\right|^{2}$ varies with $T$, the solution of (4.97) requires a $T$-dependent $\mu \neq 0$. This approach fails in the canonical ensemble for $0<T<T_{c}$, since instead of $\mu$, there is only the total density (which is different from $\left|\sigma_{0}\right|^{2}$ ) as the independent parameter; without the correct zeroth order $\sigma_{0}$, the expansion (4.92) cannot even begin.

Therefore, we should stay with the grand canonical ensemble in order to have the average

$$
\lim _{i \rightarrow 0} \lim _{\Omega \rightarrow \infty}[\phi] \neq 0
$$

at $0<T<T_{\text {c }}$ (for $D=1$ and 2, $T_{c}=0$ ). Note that $[\phi(\boldsymbol{x})]$ is independent of $\boldsymbol{x}$, and therefore $[\phi]=\sigma_{0}$.

To conclude, we can use the canonical average statement $(4.87)$ as a criterion for the soliton liquid-gas condensation (phase transition due to the translational symmetry breaking of $\phi \phi$ ), and the grand canonical average condition (4.106) for the BE condensation (phase transition due to the $U(1)$ symmetry breaking of $\phi$ ). In the case of helium, the former is the transition between HeI and the helium gas, and the latter is the $\Lambda$-transition between HeI and HeII. The critical temperature of the soliton condensation can be nonzero if $D \geq 2$, whereas the BE transition has a nonzero critical temperature only for $D \geq 3$.

\section{Friedberg-Lee model for hadrons}

\subsection{General discussion}

QCD is believed to be the theory for the strong interaction. It describes the interactions between quarks and gluons (the building blocks of all hadrons), and is highly nonlinear. At very high energies. the effective coupling constant is small due to a property shared by all such non-Abelian gauge theories, called asymptotic freedom. Comparison with experiment and perturbation expansion at high energies has verified many aspects of the theory. But at low energy, the effective coupling is not small, and nonperturbative effects dominate. As yet one is still not able to derive the structure of hadrons in terms of QCD. Friedberg and Lee used a phenomenological scalar field to model the nonperturbative part of the theory at low energies; it is often called the Friedberg-Lee model [17-20]. In this model, hadrons appear as nontopological solitons.

Let us first write down the Lagrangian density of QCD (in units $\hbar=c=1$ ),

$$
\mathscr{L}_{\mathrm{OCD}}=\bar{\psi}\left(\mathrm{i} \gamma^{\mu} D_{\mu}-m\right) \psi-{ }_{4}^{1} F_{\mu \mu^{\prime}}^{c} F_{c}^{\mu \mu^{\prime \prime}} \text {. }
$$

where for each flavor (for simplicity, we suppress the flavor index)

$$
\psi=\left(\begin{array}{l}
\psi^{1} \\
\psi^{2} \\
\psi^{3}
\end{array}\right)
$$


with $\psi^{i}$ a four-component Dirac spinor $(i=1,2,3)$,

$$
F_{\mu \nu}^{c}=\partial_{\mu} A_{\nu}^{c}-\partial_{\nu} A_{\mu}^{c}+g f_{a b}^{c} A_{\mu}^{a} A_{\nu}^{b}, \quad \partial_{\mu}=\partial / \partial x^{\mu}, \quad D_{\mu}=\partial_{\mu}-\mathrm{i} g \frac{1}{2} \lambda_{c} A_{\mu}^{c} .
$$

The $\lambda_{c}$ are the color Gell-Mann SU(3) matrices. The subscripts and superscripts $a, b, c$ run from 1 to 8 . It is customary to write

$$
\begin{array}{ll}
\lambda_{1}=\left(\begin{array}{lll}
0 & 1 & 0 \\
1 & 0 & 0 \\
0 & 0 & 0
\end{array}\right), & \lambda_{2}=\left(\begin{array}{ccc}
0 & -\mathrm{i} & 0 \\
\mathrm{i} & 0 & 0 \\
0 & 0 & 0
\end{array}\right), \quad \lambda_{3}=\left(\begin{array}{rrr}
1 & 0 & 0 \\
0 & -1 & 0 \\
0 & 0 & 0
\end{array}\right), \quad \lambda_{4}=\left(\begin{array}{lll}
0 & 0 & 1 \\
0 & 0 & 0 \\
1 & 0 & 0
\end{array}\right), \\
\lambda_{5}=\left(\begin{array}{lll}
0 & 0 & -\mathrm{i} \\
0 & 0 & 0 \\
\mathrm{i} & 0 & 0
\end{array}\right), & \lambda_{6}=\left(\begin{array}{lll}
0 & 0 & 0 \\
0 & 0 & 1 \\
0 & 1 & 0
\end{array}\right), \quad \lambda_{7}=\left(\begin{array}{ccc}
0 & 0 & 0 \\
0 & 0 & -\mathrm{i} \\
0 & \mathrm{i} & 0
\end{array}\right), \quad \lambda_{8}=\frac{1}{\sqrt{3}}\left(\begin{array}{ccc}
1 & 0 & 0 \\
0 & 1 & 0 \\
0 & 0 & -2
\end{array}\right) .
\end{array}
$$

They satisfy the relationships

$$
\left[\lambda_{a}, \lambda_{b}\right]=2 \mathrm{i} f_{a b c} \lambda_{c}, \quad\left\{\lambda_{a}, \lambda_{b}\right\}=\frac{4}{3} \delta_{a b}+2 d_{a b c} \lambda_{c}, \quad \operatorname{Tr} \lambda_{a} \lambda_{b}=2 \delta_{a b} .
$$

The nonzero elements of $f_{a b c}$ and $d_{a b c}$ are listed in table 1 .

The Lagrangian density of the Friedberg-Lee model is given by

$$
\mathscr{L}=\mathscr{L}_{\mathrm{q}}+\mathscr{L}_{\sigma}+\mathscr{L}_{\mathrm{q} \sigma}+\mathscr{L}_{\mathrm{G}}
$$

where

$$
\mathscr{L}_{\mathrm{q}}=\bar{\psi}\left(\mathrm{i} \gamma^{\mu} D_{\mu}-m\right) \psi
$$

Table 1

Nonzero elements of $f_{i m n}$ and $d_{i m n}$.

\begin{tabular}{cccc}
\hline$l m n$ & $f_{m n}$ & $l m n$ & $d_{l m n}$ \\
\hline 123 & 1 & 118 & $1 / \sqrt{3}$ \\
147 & $\frac{1}{2}$ & 146 & $\frac{1}{2}$ \\
156 & $-\frac{1}{2}$ & 157 & $\frac{1}{2}$ \\
246 & $\frac{1}{2}$ & 228 & $1 / \sqrt{3}$ \\
257 & $\frac{1}{2}$ & 247 & $-\frac{1}{2}$ \\
345 & $\frac{1}{2}$ & 256 & $\frac{1}{2}$ \\
367 & $-\frac{1}{2}$ & 338 & $1 / \sqrt{3}$ \\
458 & $\frac{1}{2} \sqrt{3}$ & 344 & $\frac{1}{2}$ \\
678 & $\frac{1}{2} \sqrt{3}$ & 355 & $\frac{1}{2}$ \\
& & 366 & $-\frac{1}{2}$ \\
& & 377 & $-\frac{1}{2}$ \\
& & 448 & $-1 / 2 \sqrt{3}$ \\
& & 558 & $-1 / 2 \sqrt{3}$ \\
& & 668 & $-1 / 2 \sqrt{3}$ \\
& & 778 & $-1 / 2 \sqrt{3}$ \\
& & 888 & $-1 / \sqrt{3}$ \\
\hline
\end{tabular}


is the Dirac term for the quarks, the scalar field $\sigma$-part is

$$
\mathscr{L}_{\sigma}=\frac{1}{2}\left(\partial^{\mu} \sigma\right)\left(\partial_{\mu} \sigma\right)-U(\sigma)
$$

the third term is the quark- $\sigma$ coupling

$$
\mathscr{L}_{\mathrm{q} \sigma}=-f \sigma \bar{\psi} \psi
$$

and the last term

$$
\mathscr{L}_{\mathrm{G}}=-\frac{1}{4} \kappa(\sigma) F_{c}^{\mu \prime} F_{\mu u^{\prime}}^{c}
$$

is the gluon part of the Lagrangian. In the absence of $\mathscr{L}_{t r}$ and $\mathscr{L}_{\mathrm{q} \sigma}$, and when $\kappa(\sigma)=1$, the Lagrangian density (5.6) reduces to that of the standard QCD expression (5.1).

The nonlinear self-interaction of the $\sigma$ field $U(\sigma)$ is usually chosen to have the quartic form

$$
U(\sigma)=\frac{1}{2 !} a \sigma^{2}+\frac{1}{3 !} b \sigma^{3}+\frac{1}{4 !} c \sigma^{4}+p
$$

with its coefficients chosen such that it has the shape of the dashed curves $(p>0$ and $p=0)$ in fig. 14 . There are two minima: an absolute minimum at a large value $\sigma$, and another at $\sigma=0$. The former corresponds to the physical vacuum, with $\sigma=\sigma_{v}$ representing some condensate. The other represents a metastable vacuum (perturbative vacuum) in which the condensate vanishes, with an energy density $p$ relative to the physical vacuum (i.e. $p$ is adjusted to make $U\left(\sigma_{v}\right)=0$ ).

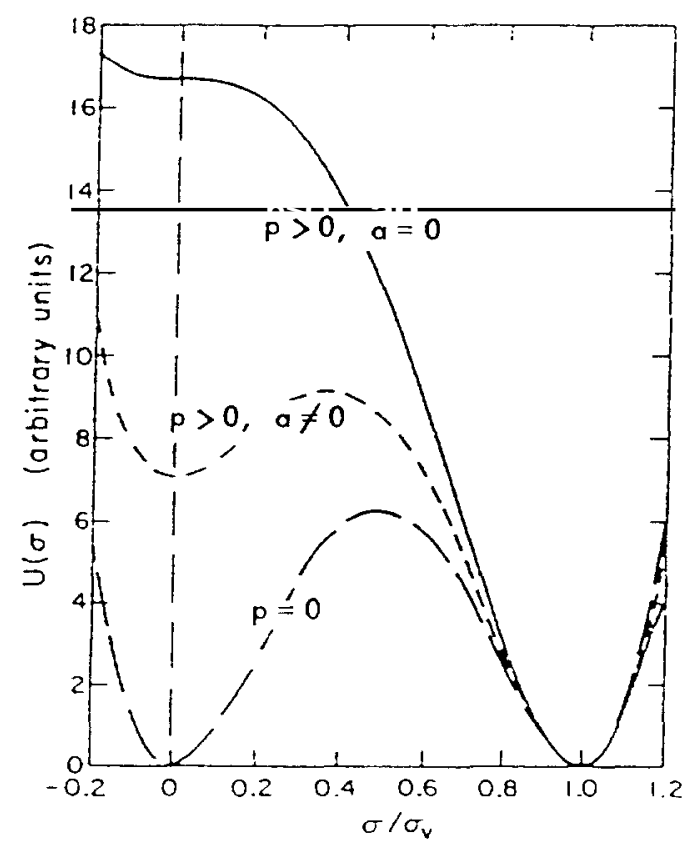

Fig. 14. Three typical forms for the potential $U(\sigma)$ in the soliton bag model. 
The Friedberg-Lee model has soliton solutions even without the gluon part of the Lagrangian. The coupling between the scalar field and the fermion field $\mathscr{L}_{q \sigma}$ acts like a mass term for the quarks. Hence (for $m=0$ ) in the physical vacuum, $\sigma=\sigma_{v}$, all quarks have a large mass $f \sigma_{\mathrm{v}}$ which makes it energetically unfavourable for them to exist there. In contrast, in a region of the metastable vacuum the quarks have zero rest mass and so only kinetic energies. However, the situation is altered when there is a nonvanishing quark density. In that case, the coupling $\mathscr{L}_{\mathrm{q} \sigma}$ provides an additional linear driving term in the potential for the $\sigma$ field. This tends to increase the energy of the physical vacuum relative to the metastable one. If the density is high enough it can raise the energy of the physical vacuum sufficiently to make the perturbative vacuum stable, at least locally. Hence we have the possibility of trapping quarks in a finite region of the metastable vacuum, where the quark density provides a force keeping the $\sigma$ field close to zero. The conserved quantity here is the fermion number. Just like the scalar soliton in three dimensions, when the fermion number $N$ is sufficiently large, the soliton solution exists, and is stable. A classical solution is presented in section 5.2.

The dielectric function $\kappa(\sigma)$ satisfies the following conditions in order to guarantee color confinement:

$$
\kappa(0)=1, \quad \kappa\left(\sigma_{\mathrm{v}}\right)=0, \quad \kappa^{\prime}\left(\sigma_{\mathrm{v}}\right)=0
$$

where $\kappa^{\prime}=\mathrm{d} \kappa / \mathrm{d} \sigma$. Because the velocity of light $c=1$ (in vacuum and in our units), the product of the color dielectric constant $\kappa$ and the color magnetic susceptibility $\mu$ must be 1 . As $\kappa \rightarrow 0$, the physical vacuum becomes a perfect color dia-electric; since $\mu=\kappa^{-1} \rightarrow \infty$, the physical vacuum is also a perfect color "anti-diamagnet".

In the immediate vicinity of quarks, antiquarks and gluons there must be a color electric field. The perfect color dia-electric property of the QCD vacuum forces the color electric field back towards its source, making it form clusters of color singlets (hadrons). This is analogous to the Meissner effect exhibited by a superconductor in QED. The superconductor is a perfect diamagnet, which expels the magnetic field outward. The QCD vacuum is a perfect color dia-electric, and leads to color confinement. In a superconductor, magnetic fields can nevertheless penetrate its surface (on the scale of the London length) or, when the field strength is sufficiently high, enter the superconductor as vortex filaments. Similar behaviour is also expected for the QCD vacuum.

To exhibit more explicitly the color confinement phenomenon consider Gauss' law (neglecting nonlinear terms)

$$
\partial \cdot D^{\mathrm{c}}=\rho^{\mathrm{c}}
$$

where the $D$-field is related to the color electric field by

$$
D^{\mathrm{c}}=\kappa E^{\mathrm{c}} \text {. }
$$

An isolated cavity containing a non-zero color charge will lead to a $D$-field which falls off as $r^{-2}$ when $r \rightarrow \infty$. The energy of the color electric field,

$$
\int \mathrm{d}^{3} r D^{\mathrm{c}} D^{\mathrm{c}} / \kappa
$$

will diverge since $\kappa(r) \rightarrow 0$ exponentially as the $\sigma$ field returns to the physical vacuum. Thus an isolated 
color object would have an infinite energy. The dia-electric still allows the color $E$ and $B$ fields to penetrate the vacuum. However, vanishing $\kappa$ in the vacuum is sufficient to guarantee that there are no long range van der Waal forces between isolated solitons. The color $D$-field between two widely separated color objects forms a flux tube. This produces a linearly rising, confining potential between them. The particular form of $\kappa(\sigma)$ is not crucial so long as the above properties are satisfied. The $U(\sigma)$ of (5.11) gives renormalizable theory, except for the presence of $\kappa(\sigma)$. However, to have color confinement, one is concerned with distance $>$, or about, a few tenths of a femtometer. The modified gluon $\mathscr{L}_{\mathrm{G}}$, given by $(5.10)$, is only a phenomenological representation, not expected to hold in the ultraviolet limit; its unrenormalizability does not present any real difficulty.

In a superconductor, the Meissner effect is the result of the spontaneous electromagnetic gaugesymmetry breaking, similar to the Higgs mechanism in the standard model of the electroweak interaction, with the $Z^{\prime \prime}$ and $W^{+}$masses replaced by the inverse of the London length $\lambda_{\mathrm{L}}$. On the other hand, in QCD the color-gauge invariance is thought to be an exact symmetry; the color-confinement is not connected with any spontaneous symmetry breaking mechanism. Yet in the Friedberg-Lee model, the color-confinement is achieved phenomenologically by representing the physical vacuum as a perfect color dia-electric, similar to the superconductor being a perfect diamagnet. Does that then mean the color symmetry is also broken spontaneously?

In the Friedberg-Lee model, for states that are not color-singlets, the color-gauge symmetry is indeed broken spontaneously. However, since these states are of infinite energy, there is no contradiction with QCD. This can be seen most directly by introducing an infinitesimal mass $\varepsilon$ to the color vector field $V_{\mu}^{\mathrm{c}}$, making its longitudinal mode physical. Taking the limit $\kappa\left(\sigma_{v}\right) \rightarrow 0$ first (and $\varepsilon \rightarrow 0$ last), the state of a single gluon does acquire an infinite mass, consistent with the spontaneous symmetry breaking mechanism. For color singlet (bag/soliton) states, since $\sigma$ approaches $\sigma_{v}$ only asymptotically at infinity, color gluons can propagate over finite distances; within any finite domain, the Lagrangian density (5.6) is color-gauge invariant.

Another interesting limit is to set $m=0$ in (5.7) and also the quark- $\sigma$ coupling $f=0$ in (5.9). In that case, the Lagrangian density $\mathscr{L}$ of the Friedberg-Lee model is invariant under the chiral transformation $[77,78]$

$$
\psi \rightarrow \gamma_{5} \psi
$$

Again, the state of a single (color) quark should have an infinite mass; in this case, the spontaneous color-gauge symmetry breaking also gives rise to a spontaneous chiral symmetry breaking. But for color singlet states, within any finite domain, $\mathscr{L}$ is chirally invariant (provided $m=f=0$ ).

Besides the possible form of $\kappa(\sigma)$, the Friedberg-Lee model has five parameters: the three constants $a, b, c$ in the $U(\sigma)$, the quark $-\sigma$ coupling constant $f$ and

$$
\alpha_{s}=g^{2} / 4 \pi
$$

the strong coupling constant. These constants are currently chosen to fit and predict the following data: - the nucleon mass $m_{\mathrm{N}}=939 \mathrm{MeV}$,

- the proton root-mean-square charge radius $\left\langle r_{\mathrm{p}}^{2}\right\rangle^{1 / 2}=0.83 \mathrm{fm}$,

- the nucleon gyromagnetic ratios, $\mu_{\mathrm{p}}=2.7928$ and $\mu_{n}=-1.9130$,

- the ratio of the axial to vector coupling constants $g_{\mathrm{A}} / g_{\mathrm{V}} \simeq 1.26$.

- the light meson masses, $m_{\pi}=138 \mathrm{MeV}, m_{p} \sim m_{(0)} \sim 780 \mathrm{MeV}$, 
- the delta-nucleon mass splitting $m_{\Delta}-m_{\mathrm{N}}=279 \mathrm{MeV}$,

- the coefficient of the linear term in the heavy quark-antiquark potential, or the string tension,

- the glueball mass.

\subsection{Classical solutions}

We shall first introduce the classical solution of the Friedberg-Lee model without the gluon fields. The equations of motion derived from the Lagrangian density (5.6) are (for $m=0$ )

$$
\begin{aligned}
& (\mathrm{i} \not \partial-f \sigma) \psi=0, \\
& \square \sigma+\mathrm{d} U / \mathrm{d} \sigma+f \bar{\psi} \psi=0,
\end{aligned}
$$

where

$$
\not \partial=\gamma^{\mu} \partial_{\mu}, \quad \square=g^{\mu \nu} \partial_{\mu} \partial_{\nu} .
$$

For a spherically symmetric $\sigma(\boldsymbol{r})$, the c number Dirac function can be written as

$$
\psi_{\lambda}=\mathrm{e}^{-\mathrm{i} \varepsilon_{\lambda} t}\left(\begin{array}{c}
u_{\lambda}(r) \\
\mathrm{i} \boldsymbol{\sigma} \cdot \hat{r} v_{\lambda}(r)
\end{array}\right) \mathscr{Y}_{\lambda m}^{\prime}
$$

where $\boldsymbol{\sigma}$ are $2 \times 2$ Pauli matrices, $\mathscr{Y}_{\lambda m}$ is the standard two-component Pauli spinor harmonic,

$$
\mathscr{Y}_{\lambda m}^{l}=\sum_{m_{l} m_{s}}\left\langle l m_{l} \frac{1}{2} m_{s} \mid j m\right\rangle Y_{l m_{l}} \chi_{m_{s}}
$$

$\hat{\boldsymbol{r}}=\boldsymbol{r} / \boldsymbol{r}$ and $\lambda$ is the Dirac quantum number,

$$
\lambda=\left(j+\frac{1}{2}\right)(-1)^{j-l+1 / 2} .
$$

Equations (5.18)-(5.22) become

$$
\begin{aligned}
& \left(\frac{\mathrm{d}}{\mathrm{d} r}+\frac{\lambda+1}{r}\right) u_{\lambda}+\left(f \sigma+\varepsilon_{\lambda}\right) v_{\lambda}=0,\left(\frac{\mathrm{d}}{\mathrm{d} r}-\frac{\lambda-1}{r}\right) v_{\lambda}+\left(f \sigma-\varepsilon_{\lambda}\right) u_{\lambda}=0, \\
& -\frac{1}{r^{2}} \frac{\mathrm{d}}{\mathrm{d} r}\left(r^{2} \frac{\mathrm{d} \sigma}{\mathrm{d} r}\right)+\frac{\mathrm{d} U}{\mathrm{~d} \sigma}+\frac{f}{4 \pi} \sum\left(u_{\lambda}^{2}-v_{\lambda}^{2}\right)=0,
\end{aligned}
$$

with the normalization condition

$$
\int_{0}^{\infty} r^{2} \mathrm{~d} r\left(u_{\lambda}^{2}+v_{\lambda}^{2}\right)=1 .
$$

In the last equation of (5.24) the sum is over all valence quarks. There are three unknowns in (5.24), the energy $\varepsilon_{\lambda}, \sigma(0)$ and $u_{\lambda}(0)$. (Note that $v_{\lambda}(0)=0$.) They are fixed by the boundary conditions at $\infty$. At $r=\infty$, 


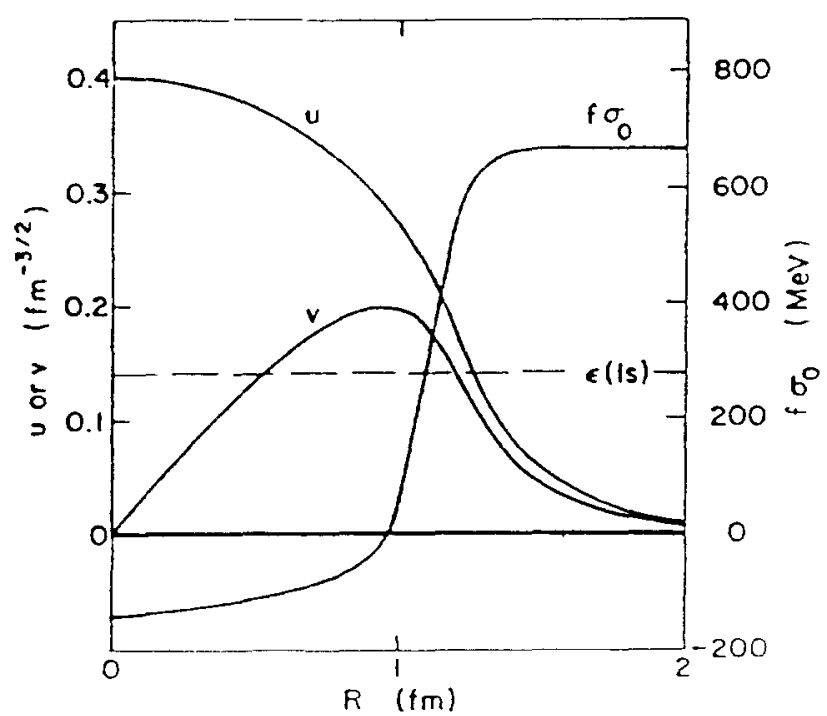

Fig. 15. The quark wave functions $u, v$ and the scalar field $\sigma$ for a ground state baryon with three valence quarks.

$$
\sigma=0, \quad u_{\lambda}=v_{\lambda}=0
$$

Equations (5.24) can be solved numerically [79-82].

For a ground state baryon with three valence s-state quarks $(\lambda=-1)$, the solution is shown in fig. 15. The total energy of this system is

$$
E=3 \varepsilon_{-1}+4 \pi \int \mathrm{d} r r^{2}\left[\frac{1}{2}(\mathrm{~d} \sigma / \mathrm{d} r)^{2}+U(\sigma)\right]
$$

The mean-square charge radius of the proton is given by

$$
\left\langle r_{\mathrm{p}}^{2}\right\rangle=\int \mathrm{d} r r^{4}\left(u_{-1}^{2}+v_{-1}^{2}\right)
$$

and its gyromagnetic ratio by'

$$
\mu_{\mathrm{p}}=\frac{2}{3} \int \mathrm{d} r r^{3} u_{-1} v_{-1}
$$

In this simple model, the neutron magnetic moment is $-2 / 3$ that of the proton, and its charge radius is zero. The nucleon axial coupling, measured in neutron decay, is given by

$$
\frac{g_{\mathrm{A}}}{g_{\mathrm{V}}}=\frac{5}{3} \int \mathrm{d} r r^{2}\left(u_{-1}^{2}-\frac{1}{3} v_{-1}^{2}\right)
$$

The wave function of a baryon is constructed by antisymmetrizing the quark wavefunctions. The result must be a color singlet. Details are given by Wilets [20]. 
When gluons are included in the full Lagrangian (5.7), the equations of motion are

$$
\begin{aligned}
& {\left[\mathrm{i} \gamma^{\mu} D_{\mu}-f \sigma\right] \psi=0, \quad \square \sigma+\frac{\mathrm{d} U}{\mathrm{~d} \sigma}+\frac{\mathrm{d} \kappa(\sigma)}{\mathrm{d} \sigma} \frac{1}{4} F_{\mu \nu}^{c} F_{c}^{\mu \nu}+f \bar{\psi} \psi=0,} \\
& \partial^{\mu} \kappa(\sigma) F_{\mu \nu}^{c}=g \bar{\psi} \frac{1}{2} \gamma_{\nu} \lambda^{c} \psi-g \kappa(\sigma) f_{a b}^{c}\left(A^{\mu}\right)^{c} F_{\mu \nu}^{b}, \quad \text { where } \lambda^{c}=\lambda_{c}, \quad f_{a b}^{c}=f_{a b c} .
\end{aligned}
$$

In the one-gluon exchange approximation the non-Abelian terms are neglected. The gluon propagation is then similar to QED in the presence of an inhomogeneous dielectric medium. The calculation of the gluon Green's function for arbitrary spatial dependence of the dielectric can be carried out. It is usually convenient to work in the transverse gauge

$$
\boldsymbol{\partial} \cdot \boldsymbol{\kappa}=0
$$

The scalar Green's function then satisfies the time-independent equation

$$
\partial \cdot \kappa(r) \partial G^{00}\left(r, r^{\prime}\right)=-\delta\left(r-r^{\prime}\right)
$$

The space-tensor Green's function has to be transverse due to the gauge condition, and its time-Fourier transform satisfies

$$
\left(\omega^{2}+\partial^{2}\right) \kappa(\boldsymbol{r}) G^{i j}\left(r, \boldsymbol{r}^{\prime} ; \omega\right)-\varepsilon_{i k l} \partial_{k}\left[\varepsilon_{l m n} \kappa(\boldsymbol{r}) G^{m j}\left(\boldsymbol{r}, \boldsymbol{r}^{\prime} ; \omega\right) \partial_{n} \ln \kappa(\boldsymbol{r})\right]=-\delta_{\mathrm{T}}^{i j}\left(\boldsymbol{r}, \boldsymbol{r}^{\prime}\right)
$$

where $\varepsilon_{i j k}=+1$ (or -1 ) if $i j k$ is an even (or odd) permutation of $1,2,3$ and zero otherwise,

$$
\delta_{\mathrm{T}}^{i j}\left(\boldsymbol{r}, \boldsymbol{r}^{\prime}\right)=\delta^{i j} \delta\left(\boldsymbol{r}-\boldsymbol{r}^{\prime}\right)+\kappa(\boldsymbol{r}) \partial_{i} \partial_{j}^{\prime} G^{00}\left(\boldsymbol{r}, \boldsymbol{r}^{\prime}\right)
$$

is the transverse $\delta$-function. These Green's functions are given by Lee [83] and by Tang and Wilets [84].

\subsection{Mean field approximation}

Without the gluon field, the solution of the Friedberg-Lee model is very similar to that of the MIT bag model [11]. In the MIT bag model the quarks are confined in an infinite square-well scalar potential. With a soliton model, the quarks are confined dynamically by their interactions with another field. The advantage of this approach is that the theory can be quantized in a straightforward manner; also, the relativistic invariance is manifest. The Hamiltonian and its density are

$$
\begin{aligned}
& H=\int \mathrm{d}^{3} r \mathscr{H}, \\
& \mathscr{H}=\psi^{\dagger}[-\mathrm{i} \boldsymbol{\alpha} \cdot \boldsymbol{\partial}+g \beta \sigma] \psi+\frac{1}{2} \pi^{2}+\frac{1}{2}(\boldsymbol{\partial} \sigma)^{2}+U(\sigma),
\end{aligned}
$$

where $\pi$ is the conjugate momentum of the $\sigma$ field,

$$
\pi=\partial_{0} \sigma
$$


We can separate the sigma field according to

$$
\sigma=\sigma_{0}(\boldsymbol{r})+\sigma_{1}, \quad \pi=\pi_{0}(\boldsymbol{r})+\pi_{1},
$$

where $\sigma_{0}$ is the time-independent $\mathrm{c}$ number $\sigma$-function obtained in the previous section, $\sigma$ is the quantum fluctuation and $\pi_{0}=0$. These operators satisfy the usual equal time commutation relations for bosons

$$
\left[\pi(\boldsymbol{r}, t), \sigma\left(\boldsymbol{r}^{\prime}, t\right)\right]=\left[\pi_{1}(\boldsymbol{r}, t), \sigma_{1}\left(\boldsymbol{r}^{\prime}, t\right)\right]=-\mathrm{i} \delta^{3}\left(\boldsymbol{r}-\boldsymbol{r}^{\prime}\right)
$$

Similarly, we can represent the quark operator by

$$
\psi=\sum_{k} c_{k}(t) \psi_{k}(r)
$$

where $c_{k}$ satisfy the equal time anti-commutation relation for fermions

$$
\left\{c_{k}, c_{k}^{\dagger}\right\}=\delta_{k k}
$$

and the $\psi_{k}$ are any complete and orthonormal set of spinor-color-flavor functions. For a fixed number of valence quarks, the Hamiltonian density can be expanded in terms of $\sigma_{1}$

$$
\mathscr{H}=\mathscr{H}+\mathscr{H}^{\prime} \sigma_{1}+\frac{1}{2} \mathscr{H}^{\prime} \sigma_{1}^{2}+\frac{1}{6} \mathscr{H}^{\prime \prime} \sigma_{1}^{3}+\frac{1}{24} \mathscr{H}^{\prime \prime \prime} \sigma_{1}^{4},
$$

where $\mathscr{H}$ and its derivatives $\mathscr{H}^{\prime}, \mathscr{H}^{\prime \prime}, \ldots$ are evaluated at $\sigma_{0}$. Varying the expectation value of $H$ with respect to $\psi_{k}$ and $\sigma_{1}$, and neglecting terms of order $\sigma_{1}^{2}$ and higher in $H$, we have the classical equations of motion (5.18) and (5.19).

In terms of the quark state obtained from classical equations, we can rewrite the Hamiltonian,

$$
\begin{aligned}
H= & E_{0}+\sum_{k} \varepsilon_{k} c_{k}^{*} c_{k}+\int \mathrm{d}^{3} r\left(\frac{1}{2}\left(\pi_{1}^{2}+\left|\boldsymbol{\partial} \sigma_{1}\right|^{2}+U^{\prime \prime} \sigma_{1}^{2}\right)+\frac{1}{6} U^{\prime \prime \prime} \sigma_{l}^{3}+\frac{1}{24} c \sigma_{l}^{4}\right. \\
& \left.+f \sum_{k l} \bar{\psi}_{k}(\boldsymbol{r}) \sigma_{1} \psi_{l}(\boldsymbol{r}) c_{k}^{*} c_{l}\right)
\end{aligned}
$$

where $E_{0}$ is the energy of the classical field $\sigma_{0}$ only. The quantum part of the soliton field can be expanded in terms of any orthonormal set $\left\{s_{n}(r)\right\}$ :

$$
\sigma_{1}=\sum_{n}\left(2 \omega_{n}\right)^{-1 / 2}\left(a_{n}^{\dagger} s_{n}^{*}+a_{n} s_{n}\right) . \quad \pi_{1}=\sum_{n} \mathrm{i}\left(\omega_{n} / 2\right)^{1 / 2}\left(a_{n}^{\dagger} s_{n}^{*}-a_{n} s_{n}\right) .
$$

The Hamiltonian simplifies if we choose $s_{n}$ and $\omega_{n}$ to be the solution of

$$
\left(-\boldsymbol{\partial}^{2}+U^{\prime \prime}-\omega_{n}^{2}\right) s_{n}(\boldsymbol{r})=0 .
$$

We have

$$
H=E_{0}+\sum_{k} \varepsilon_{k} c_{k}^{\ddot{c}} c_{k}+\sum_{n} \omega_{n}\left(a_{n}^{\dagger} a_{n}+\frac{1}{2}\right)+H_{1}+H_{3}+H_{4},
$$


where

$$
\begin{aligned}
& H_{1}=f \sum_{k l n} \int \bar{\psi}_{k} s_{n} \psi_{l} \mathrm{~d}^{3} r\left(2 \omega_{n}\right)^{-1 / 2} c_{k}^{\dagger} c_{l} a_{n}+\text { h.c. }, \\
& H_{3}=\frac{1}{6} \int\left(b+c \sigma_{0}\right) \sigma_{1}^{3} \mathrm{~d}^{3} r, \quad H_{4}=\frac{1}{24} c \int \sigma_{1}^{4} \mathrm{~d}^{3} r .
\end{aligned}
$$

The diagrams representing $H_{1}, H_{3}$ and $H_{4}$ are shown in fig. 16, where the solid lines are quark propagators and the wavy lines are $\sigma_{1}$ propagators. The lowest normal modes of $s_{n}$ can be shown to be surface modes. They can be interpreted as meson clouds surrounding a baryon.

In the mean field approximation, since the soliton is localized in space, it does not have a definite momentum. It can be shown that, for a soliton solution, the expectation value of the total momentum operator $\langle\boldsymbol{P}\rangle=0$, while $\left\langle\boldsymbol{P}^{2}\right\rangle \neq 0$, where $\boldsymbol{P}$ is given by

$$
\boldsymbol{P}=-\frac{1}{2} \int \mathrm{d}^{3} r\left(\mathrm{i} \boldsymbol{\partial}\left(\psi^{\dagger} \psi\right)+\{\pi, \boldsymbol{\partial} \sigma\}\right)
$$

This gives rise to spurious center-of-mass energies and center-of-mass fluctuational motions. One can correct this problem within the mean field approximation by defining [85]

$$
m=\sqrt{\langle H\rangle^{2}-\left\langle P^{2}\right\rangle}
$$

and identifying $m$ with the mass of a soliton, referred to as the "recoil-corrected mass". The quark distribution radius squared of a soliton is defined as

$$
\left\langle r^{2}\right\rangle=\left\langle\left|\left(\sum_{i=1}^{N} r_{i}\right)^{2}\right|\right\rangle
$$

where $r_{i}$ is the position operator for the $i$ th quark, $N$ is the total number of quarks and $\mid>$ is the quantum state. If we approximate the expectation value of an operator $\mathcal{O}$ by

$$
\langle O\rangle=\frac{1}{E} \int \mathrm{d}^{3} r\langle|O(\boldsymbol{r}) H(\boldsymbol{r})|\rangle, \text { where } E=\int \mathrm{d}^{3} r\langle|H(\boldsymbol{r})|\rangle,
$$

then the quark distribution radius squared, (5.50), for a baryon (with three valence quarks) is

$$
\left\langle r^{2}\right\rangle=\left(1-2 \varepsilon / E+3 \varepsilon^{2} / E^{2}\right)\left\langle r_{1}^{2}\right\rangle+3 \varepsilon^{2} / 2 E^{2},
$$

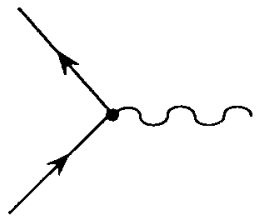

$\mathrm{H}_{1}$

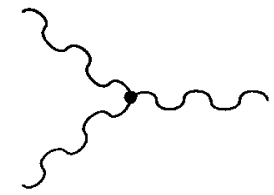

$\mathrm{H}_{3}$

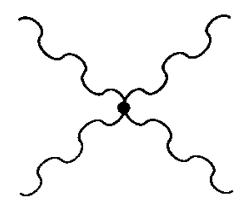

$\mathrm{H}_{4}$

Fig. 16. Interaction terms $H_{1}, H_{3}$ and $H_{4}$, where the solid lines are quark propagators and the wavy lines are $\sigma_{1}$ propagators. 
Table 2

\begin{tabular}{|c|c|c|c|c|}
\hline$\left.\underline{a(\mathrm{fm}}{ }^{2}\right)$ & $\underline{-b}(\mathrm{fm})$ & $\underline{c}$ & I & $\underline{D}\left(\mathrm{MeV} / \mathrm{fm}^{\mathrm{i}}\right)$ \\
\hline 0.0000 & 14.66 & $1(H)$ & 10.531 & 10.25 \\
\hline 7.671 & 107.27 & 500 & 13.088 & 0.00 \\
\hline 1.602 & 69.33 & $5(11)$ & 9.572 & 16.85 \\
\hline 0.000 & 58.52 & 500 & 9.158 & 20.83 \\
\hline 12.849 & 196.34 & 1,000 & 13.064 & 0.00 \\
\hline 2.589 & 124.64 & $1 .(0) 0$ & 9.361 & 22.01 \\
\hline 0.000 & 105.14 & 1,0001 & 9.037 & 27.12 \\
\hline $4(1.880$ & 783.08 & 5,000 & 14.014 & 0.00 \\
\hline 7.510 & 474.66 & 5,000 & 10.092 & 37.03 \\
\hline 0.000$)$ & 399.10 & 5.000 & 10.010 & 45.16 \\
\hline 66.422 & 1.411 .60 & $10.000)$ & 14.832 & 0.00 \\
\hline 11.605 & 834.44 & 10.0000 & 10.957 & 44.21 \\
\hline 0.0000 & 700.43 & 10.0000 & 10.977 & 53.43 \\
\hline 321.750 & 9.824 .8 & 100,000 & 19.770 & 0.00 \\
\hline 45.214 & 5.208 .5 & 100,0000 & 16.379 & 67.11 \\
\hline 0.000 & 4.356 .9 & 100,0000 & 16.715 & 80.07 \\
\hline
\end{tabular}

where $\left\langle r_{1}^{2}\right\rangle$ can refer to any one of the quarks in the soliton, and $\varepsilon$ is the eigenenergy for the s-wave quark state.

Since the strong coupling constant $\alpha_{s}=0$ in the mean field approximation, we have four parameters. We may choose two constraints on these parameters so that the proton mass (computed with (5.49)) and the proton rms charge radius (computed with (5.52)) fit the experimental values exactly. The variations of the four parameters $a, b, c$ and $f$ under these two constraints are shown in table 2 .

\subsection{Beyond mean field approximation}

In order for the eigenstate of energy to be the eigenstate of total momentum, we must isolate the collective motion. The quantization procedure described in section 3.3 can be used to quantize the Friedberg-Lee soliton. Since the Hamiltonian and momentum operators commute, an eigenstate of the energy is automatically an eigenstate of the total momentum. Any quantization procedure that explicitly separates the degrees of freedom associated with collective motions from the vibrational degrees of freedom will not have the spurious center of mass motion shown in the last section. However, these quantization methods are usually very complicated to carry out exactly. In practice, some approximate schemes are often used. The methods widely used in solving the Friedberg-Lee model are the Peierls-Yoccoz projection [86] and the closely related generator coordinates method $[87,88,89]$. The latter is applied to soliton-soliton collisions $[90,91]$.

For a given state vector of a soliton, $|\boldsymbol{X}\rangle$, localized at a spatial point $\boldsymbol{X}$, we can parallelly transport it to another point $\boldsymbol{X}^{\prime}$,

$$
\left|\boldsymbol{X}^{\prime}\right\rangle=\exp \left[-\mathrm{i}\left(\boldsymbol{X}^{\prime}-\boldsymbol{X}\right) \cdot \boldsymbol{P}\right]|\boldsymbol{X}\rangle \text {. }
$$


An eigenstate of the momentum can be constructed from a linear superposition of these localized soliton states,

$$
|p\rangle=\int \mathrm{d}^{3} X \mathrm{e}^{\mathrm{i} p \cdot X}|\boldsymbol{X}\rangle
$$

This is called the Peierls-Yoccoz projection. A zero momentum projected state is simply

$$
|p=0\rangle=\int \mathrm{d}^{3} X|X\rangle
$$

An expectation value of an operator $\mathcal{O}$ in the projected state is

$$
\langle\mathcal{O}\rangle=\frac{\int \mathrm{d}^{3} X \mathrm{~d}^{3} X^{\prime}\left\langle\boldsymbol{X}|\mathcal{O}| \boldsymbol{X}^{\prime}\right\rangle}{\int \mathrm{d}^{3} X \mathrm{~d}^{3} X^{\prime}\left\langle\boldsymbol{X} \mid \boldsymbol{X}^{\prime}\right\rangle} .
$$

If the operator $\mathcal{O}$ is translationally invariant, we can introduce $\boldsymbol{Z}=\boldsymbol{X}-\boldsymbol{X}^{\prime}$. Then the expectation value of $\mathcal{O}$ can be written as

$$
\langle\mathscr{O}\rangle=\frac{\int \mathrm{d}^{3} Z\left\langle-\frac{1}{2} Z|\mathscr{O}| \frac{1}{2} Z\right\rangle}{\int \mathrm{d}^{3} Z\left\langle-\frac{1}{2} Z \mid \frac{1}{2} Z\right\rangle} .
$$

The mass of a soliton in this approximation (called the "projected" soliton mass) is then

$$
m=\langle H\rangle=\frac{\int \mathrm{d}^{3} Z\left\langle-\frac{1}{2} Z|H| \frac{1}{2} Z\right\rangle}{\int \mathrm{d}^{3} Z\left\langle-\frac{1}{2} Z \mid \frac{1}{2} Z\right\rangle} .
$$

In fig. 17, we compare the baryon mass of a "projected" soliton, as a function of the model parameter $c$, with the "recoil-corrected" mass (5.49) and that calculated from the mean field approximation.

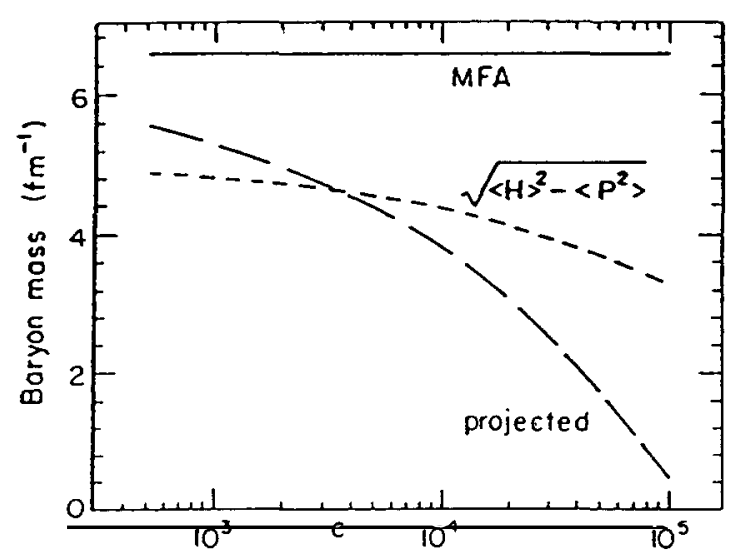

Fig. 17. Mass of a bag for one family of parameters $\left(b^{2} / a c\right)$ and varying $c$. The solid line is from the mean field approximation, the short dashed line is the mean field approximation with recoil correction, and the long dashed curve is calculated after a projection. 
In principle, $|\boldsymbol{X}\rangle$ can be a solution under the mean field approximation, (5.39), where the $\sigma$-field is separated into a c number part $\sigma_{0}$ and a quantum fluctuation $\sigma_{1}$. Because the operator $\sigma_{1}$ is defined with respect to $\sigma_{0}$, the matrix elements connecting states of different $\sigma_{0}$ are not easily calculable. To avoid the use of the spatially dependent mean field, we can construct the $\sigma$ part of the $|\boldsymbol{X}\rangle$ to be a coherent state [92-95]. We follow Lubeck et al. [95] and expand $\sigma$ and $\pi$ in some complete set of orthonormal functions,

$$
\sigma=\sigma_{v}+\sum_{n}\left(2 \omega_{n}\right)^{-1 \cdot 2}\left[a_{n}^{\dagger} s_{n}^{*}(\boldsymbol{r})+a_{n} s_{n}(\boldsymbol{r})\right], \quad \pi=\sum_{n} \mathrm{i}\left(\omega_{n} / 2\right)^{1 \cdot 2}\left[a_{n}^{*} s_{n}^{*}(\boldsymbol{r})-a_{n} s_{n}(\boldsymbol{r})\right]
$$

where $\omega_{n}$ are as yet undetermined and $s_{n}$ can be chosen to be plane waves. $\sigma$ and $\pi$ satisfy the usual equal time commutation relations if

$$
\left[a_{n}(t), a_{n^{\prime}}(t)^{\dagger}\right]=\delta_{n n^{\prime}}, \quad\left[a_{n}(t), a_{n^{\prime}}(t)\right]=\left[a_{n}(t)^{\dagger}, a_{n^{\prime}}(t)^{\dagger}\right]=0
$$

A coherent state in one mode, say $n=0$, is obtained by the construction

$$
|\lambda\rangle \equiv \exp \left(\lambda a_{0}^{\dagger}\right)|0\rangle
$$

Such a wave function was first used by Lee and Pines [96] and by Lee, Low and Pines [97] in studying polarons. It was further studied by Klauder [98] and Glauber [99], and used in quantum representations of soliton solutions by others $[67,68,100,101]$. It is straightforward to verify that $|\lambda\rangle$ is an eigenstate of the annihilation operator $a_{01}$,

$$
a_{0}|\lambda\rangle=\lambda|\lambda\rangle
$$

For any normal ordered operator of $O\left(a_{0}^{\dagger}, a_{0}\right)$,

$$
\left\langle\lambda\left|: \mathcal{O}\left(a_{0}^{\dagger}, a_{0}\right):\right| \lambda^{\prime}\right\rangle=\mathrm{e}^{\lambda^{*} \lambda} \mathscr{O}\left(\lambda^{*}, \lambda^{\prime}\right)
$$

A more complex coherent state vector can be constructed by taking a product of exponentiated operators:

$$
|\phi\rangle=\exp \left(\sum_{p}\left(\omega_{p} / 2\right)^{1 / 2} f_{p} a_{p}^{*}\right)|0\rangle \exp \left(-\frac{1}{2} \sum_{p} \omega_{p}\left|f_{p}\right|^{2}\right)
$$

where $f_{p}$ is the Fourier coefficient of $\sigma_{i 1}(r)$, and $\omega_{p}$ is to be determined. The expectation values of $\sigma$ and $\pi$ are

$$
\langle\phi|\sigma| \phi\rangle=\sigma_{\mathrm{v}}+\frac{1}{2 \Omega^{1 / 2}} \sum_{n}\left(f_{n} \mathrm{e}^{-\mathrm{i} p \cdot r}+f_{n}^{*} \mathrm{e}^{\mathrm{i} p \cdot r}\right) \equiv \sigma_{0}(\boldsymbol{r})
$$

and

$$
\langle\phi|\pi| \phi\rangle=-\frac{\mathrm{i}}{2 \Omega^{1 / 2}} \sum_{n}\left(f_{n} \mathrm{e}^{-\mathrm{i} \boldsymbol{p} \cdot \boldsymbol{r}}-f_{n}^{*} \mathrm{e}^{\mathrm{i} \boldsymbol{p} \cdot \boldsymbol{r}}\right) \equiv \pi_{0}(\boldsymbol{r}) .
$$


The total state vector, with the valence quarks, is

$$
|\Psi\rangle=\prod_{k} c_{k}^{\dagger}|\phi\rangle
$$

and the expectation value of (normal ordered) $H$ is

$$
\langle\Psi|H| \Psi\rangle=H\left(\sigma_{0}\right)
$$

Variations of $\langle\Psi|H| \Psi\rangle$ give us the same equations as in the mean field approximation.

The expectation value of $H$ after the projection depends on $\omega_{p}$ and the functions $u(r), v(r)$ and $\sigma_{0}(r)$, where $u$ and $v$ are the upper and lower components of the quark field. The mass of a soliton should be obtained by minimizing the expectation value of $H$ after the momentum projection. This is called the variation after projection. The variations with respect to all those functions appear to be prohibitively complicated at present. Instead, we can carry out variations in a limited number of variables based on the forms obtained from solving the mean field equations. Denote the mean field solution with a tilde, and introduce variational parameters $\xi, \lambda, \delta$ and $\gamma$,

$$
\begin{aligned}
& \sigma_{0}(r)-\sigma_{\mathrm{v}}=\xi\left[\tilde{\sigma}_{0}(r / \lambda)-\sigma_{\mathrm{v}}\right], \\
& u(r)=\tilde{u}(r / \delta), \quad v(r)=\gamma \tilde{v}(r / \delta) .
\end{aligned}
$$

We now discuss the comparison between experimental data and these three approximations, (i) the simple mean field approximation, (ii) the mean field approximation plus the momentum projection correction (5.58) and (iii) with the additional variations with respect to $\xi, \lambda, \delta$ and $\gamma$ (after completing (ii)). There are five parameters $a, b, c, f$ and $\alpha_{\mathrm{s}}$ in the Lagrangian (5.6)-(5.11), of which one is chosen to yield the exact proton rms charge radius $r_{\mathrm{p}}$. This leaves four free parameters. Table 3 lists the

Table 3

Nucleon and meson properties for the least-squares fit parameters with $\alpha_{\mathrm{s}}=2.25, f=5.694$ and $b^{2} / a c=5.438[95]$.

\begin{tabular}{lcccc}
\hline & (i) MFA & (ii) Proj. & (iii) Proj. + Var. & Expt. \\
\hline$m_{\mathrm{p}}(\mathrm{MeV})$ & 1218 & 1031 & 930 & 939 \\
$\left\langle r_{\mathrm{p}}^{2}\right\rangle^{1 / 2}(\mathrm{fm})$ & 0.83 & 0.83 & 0.83 & 0.83 \\
$\mu_{\mathrm{p}}$ & 2.06 & 2.96 & 2.67 & 2.76 \\
$\mu_{\mathrm{p}} / \mu_{\mathrm{n}}$ & $-2 / 3$ & $-2 / 3$ & $-2 / 3$ & -0.685 \\
$m_{\Delta}(\mathrm{MeV})$ & 1384 & 1177 & $1083^{\mathrm{a}}$ & 1232 \\
$\left\langle r_{\mathrm{s}}^{2}\right\rangle^{1 / 2}(\mathrm{fm})$ & 0.83 & - & 1.20 & - \\
$\mu_{\Delta}$ & - & - & 3.21 & - \\
$m_{\pi}(\mathrm{MeV})$ & 950 & 704 & 177 & 140 \\
$\left\langle r_{\pi}^{2}\right)^{1 / 2}(\mathrm{fm})$ & 0.71 & 0.70 & - & 0.66 \\
$f_{\pi} / m_{\pi}$ & - & - & 0.83 & 0.66 \\
$m_{\mathrm{p}}$ or $m_{\omega}(\mathrm{MeV})$ & 1103 & 840 & 723 & 770 \\
$\left\langle r_{\mathrm{p}}^{2}\right\rangle^{1 / 2}(\mathrm{fm})$ & - & - & 1.40 & 0.66 \\
$g_{\mathrm{A}} / g_{\mathrm{v}}$ & 1.21 & -1.35 & 1.27 & 1.26 \\
string tension $(\mathrm{MeV} / \mathrm{fm})$ & - & - & $910^{\mathrm{bj}}$ & $925^{\mathrm{c}}$ \\
\hline
\end{tabular}

a) Additional $\Delta-\mathrm{N}$ splitting can be accounted for by explicit coupling of pions [102].

${ }^{b)}$ Ref. [102].

${ }^{c}$ Determined from the quarkonium calculations [103-106]. 
optimal values of (i), (ii) and (iii) for the "key" experimental data mentioned in section 5.1. The result [95] is reasonably satisfactory.

There are many applications of soliton models in the study of hadronic structure and hadron-hadron interactions. Many of these solitons are variations of the Friedberg-Lee model described in this section [107-113]. A nontopological soliton model based on the linear sigma model was developed by Kahana, Ripka and Soni [114]. It contains a pion field in addition to the scalar field $\sigma$. Subsequent study by Kahana and Ripka [115] demonstrated the importance of the pion degrees of freedom. A similar model has also been studied by Birse and Banerjee [116] and by Celenza and Shakin [117]. Among topological solitons, the Skyrme model $[118,119]$ is the most widely used.

\section{Soliton stars}

\subsection{General discussion}

So far, our analysis does not include gravity. Since the existence of soliton solutions depends sensitively on the nonlinearity of the field theory and since general relativity is highly nonlinear, it is natural to inquire whether the introduction of the gravitational interaction may alter significantly the previously discussed solutions, especially when their masses become astronomically large. This problem is also of interest from another point of view: any (cold) stable star is, by definition, a nontopological soliton. Its equilibrium configuration has been calculated based on the equation of state of normal matter and the gravitational attraction. On the other hand, a major fraction of the total mass of the universe is believed to be in the form of "dark matter", of which very little is known except its existence. Now, nontopological solutions already can exist without gravity and (in that case) with no upper bound on their masses; therefore, they may be of astronomical sizes. It is reasonable to explore whether such solutions with gravity may account for structures outside our usual concept of normal stars. As we shall see, the interplay between the gravitational field and other nonlinear matter fields can lead to several novel types of stellar configurations, such as boson stars, mini-boson stars and fermion soliton stars. These solutions will be discussed in this chapter.

We begin with a brief review of the equilibrium configurations of (cold) normal stars: the solutions for white dwarfs by Chandrasekhar [120] and neutron stars by Oppenheimer and Volkoff [29].

\subsubsection{Chandrasekhar limit (review)}

Consider a white dwarf, or a neutron star, of radius $R$, mass $M$ and fermion number $N$. The gravitational force is balanced by the Fermi pressure. From the equipartition of energy we expect, for the equilibrium state, the magnitude of the gravitational energy to be comparable to that of the kinetic energy. For ultrarelativistic fermions, we have

$$
G M^{2} / R \sim N^{4 / 3} / R
$$

where $G$ is Newton's constant. Let $m$ be the effective mass, defined by

$$
N=M / m
$$

For a neutron star, the fermions are neutrons and $m$ is the neutron mass $m_{N}$; for a white dwarf, they are 
the electrons and $m=2 m_{\mathrm{N}}$, since there are two nucleons per electron. Combining (6.1) and (6.2), one sees that a critical mass $M_{\mathrm{c}}$ exists,

$$
M_{\mathrm{c}} \sim 1 / G^{3 / 2} m^{2}
$$

Relating (in units $\hbar=c=1$ ) $G=l_{\mathrm{P}}^{2}$, where $l_{\mathrm{P}}$ is the Planck length, given by $l_{\mathrm{P}} \simeq 10^{-33} \mathrm{~cm}$, we find (because $m_{\mathrm{N}}^{-1} \sim 10^{-14} \mathrm{~cm}$ )

$$
M_{\mathrm{c}} \sim m / l_{\mathrm{P}}^{3} m^{3} \sim 10^{57} m_{\mathrm{N}} \sim M_{\odot},
$$

where $M_{\odot}$ is the solar mass. For the white dwarf, this is the well-known Chandrasekhar limit, which is about $1.4 M_{\odot}$. For $M$ larger than $1.4 M_{\odot}$ but less than $M_{\mathrm{c}}$ of the neutron star, white dwarfs cease to exist: instead, one has a neutron star. For the neutron star, because of general relativity and nuclear forces, $M_{\mathrm{c}}$ is somewhat smaller than four times the white dwarf limit (as would be indicated by (6.4)); it is commonly accepted as $<5 M_{\odot}$, depending on the physical assumptions.

For $M$ larger than $M_{\mathrm{c}}$ of the neutron star, the solution becomes singular $(R=0)$. The star collapses into a black hole. This relatively low critical mass $M_{\mathrm{c}} \leq 5 M_{\odot}$ has been used as a criterion for the observation of black holes.

This critical mass $M_{\mathrm{c}}$ for stellar collapse is rather insensitive to the assumption of the equation of state. For any normal cold matter (temperature $T=0$ ) and in the usual thermodynamical limit, the pressure $p$ is a function of the density $\sim M / R^{3}$. Take, for example,

$$
p \propto\left(M / R^{3}\right)^{\gamma} .
$$

By balancing the gravitational force with the force exerted by the pressure, we have in place of $(6.1)$,

$$
G M^{2} / R^{2} \sim p R^{2} \propto\left(M / R^{3}\right)^{\gamma} R^{2},
$$

i.e.,

$$
G M^{2-\gamma} \propto R^{4-3 \gamma}
$$

To estimate gravitational collapse, we may set $R$ to be the Schwarzschild radius $2 G M$. Substituting that in $(6.5)$, we find

$$
G^{-3+3 \gamma} \propto M^{2-2 \gamma}
$$

and therefore the critical mass $M_{\mathrm{c}}$ is always proportional to $G^{-3 / 2}$, which leads again to (6.4), independent of $\gamma$. (For a relativistic Fermi gas, $\gamma=\frac{4}{3}$.)

\subsubsection{Critical masses of soliton stars [24]}

By using the nontopological soliton solutions, we can have (at least theoretically) a new type of cold stable stellar configuration, the boson or fermion soliton stars. Depending on the theory, its critical mass $M_{\mathrm{c}}$ may vary from $M_{\odot}$ to as large as $\sim 10^{12}-10^{15} M_{\odot}$. Or, in the case of mini-boson stars, $M_{\mathrm{c}}$ may be as small as $\sim 10^{10} \mathrm{~kg}$, with a density $\sim 10^{41}$ times that of a neutron star. 
To illustrate the extreme example of a very large critical mass, consider the following example of a nontopological soliton, first without gravity. The theory contains an additive quantum number $N$ (like the baryon number) carried by either a spin- $\frac{1}{2}$ field $\psi$, or spin- 0 complex field $\phi$. with its elementary field quantum having $N= \pm 1$. In addition, there is a scalar field $\sigma$. Take the self-interaction of $\sigma$ to be the typical degenerate vacuum form:

$$
U(\sigma)=\frac{1}{2} m^{2} \sigma^{2}\left(1-\sigma / \sigma_{0}\right)^{2}
$$

We may assign $\sigma=0$ to the normal vacuum state, and $\sigma=\sigma_{0}$ to the (abnormal) degenerate vacuum state. The soliton contains an interior in which $\sigma \simeq \sigma_{0}$, a shell of width $\sim m^{-1}$, over which $\sigma$ changes from $\sigma_{0}$ to 0 , and an exterior that is essentially the normal vacuum. The $N$-carrying field $\psi$, or $\phi$, is confined to the interior; this produces a kinetic energy $E_{\mathrm{k}}$ (assuming for simplicity that the mass of $\psi$, or $\phi$, is zero when $\sigma=\sigma_{0}$, but nonzero when $\sigma=0$ ):

$$
E_{\mathrm{k}} \simeq \begin{cases}(3 \pi)^{1 / 3}\left(\frac{3}{4} N\right)^{4 / 3} / R & \text { for } \psi, \\ \pi N / R & \text { for } \phi .\end{cases}
$$

The shell contains a surface energy

$$
E_{\mathrm{s}}=4 \pi s R^{2}
$$

where $s$ is the surface tension related to $\sigma_{0}$ and $m$ by

$$
s \simeq \frac{1}{6} m \sigma_{0}^{2}
$$

The radius $R$ can be calculated by minimizing the total energy $E=E_{\mathrm{h}}+E_{\varsigma}$. Setting $\partial E / \partial R=0$, we have the equipartition

$$
E_{\mathrm{k}}=2 E_{\mathrm{s}} \text {. }
$$

Hence, the soliton mass $M$ (which is the minimum of $E$ ) can be written as

$$
M=3 E_{\mathrm{s}}=12 \pi s R^{2}
$$

the total conserved quantum number is

$$
N= \begin{cases}\frac{16}{3}(2 / 3 \pi)^{1 / 4} s^{3 / 4} R^{9 / 4} & \text { for } \psi . \\ 8 s R^{3} & \text { for } \phi,\end{cases}
$$

and therefore, for large $N$,

$$
M \propto \begin{cases}N^{8 / 9} & \text { for } \psi \\ N^{2 / 3} & \text { for } \phi .\end{cases}
$$

Because the exponent of $N$ is $<1$, when $N$ is large the soliton mass is always less than that of the free particle solution, and that ensures its stability. 
Next, we include the gravitational field. For configurations with $R$ much greater than the Schwarzschild radius $2 G M$, the effects of gravity can be treated as a perturbation. Gravity becomes important when $R$ becomes of the same order as $2 G M$. Thus, the critical mass $M_{\mathrm{c}}$ may be estimated by simply equating $R$ with the Schwarzschild radius,

$$
R \sim 2 G M_{\mathrm{c}},
$$

which, because of $(6.11)$, leads to

$$
M_{\mathrm{c}} \sim\left(48 \pi G^{2} s\right)^{-1} .
$$

Set $G=l_{\mathrm{p}}^{2}$ and assume, as a typical Higgs-type field, $\sigma_{0} \sim m$ about, or higher than $30 \mathrm{GeV}$ (but much less than the Planck mass), we estimate

$$
M_{\mathrm{c}} \sim\left(l_{\mathrm{p}} m\right)^{-4} m
$$

which is of the order of a galaxy. For example, if $m \sim 30 \mathrm{GeV}$, we have $M_{\mathrm{c}} \sim 10^{15} M_{\odot}$ and $R \sim l_{\mathrm{P}}^{-2} m^{-3} \sim$ $10^{2}$ lightyear; if $m$ is $\sim 300 \mathrm{GeV}$, then $M_{\mathrm{c}} \sim 10^{12} M_{\odot}$ and $R \sim 10^{-1}$ lightyear.

At present, very little is known concerning the nature of Higgs-type bosons, except that they should be massive, spin 0 , and that their expectation values modify the masses of other fields. Thus $M_{\mathrm{c}}$ for the soliton star could also be quite different from the above estimate, depending on the theory. For example, by removing the degeneracy of the abnormal and normal vacua and adjusting their energy difference, $M_{\mathrm{c}}$ can vary from the order of a galactic mass to that of a solar mass, as we shall see.

If we take, instead of (6.6), an MIT-bag-like potential which gives the abnormal vacuum a higher potential energy density $p$ than the real vacuum, in the absence of gravity the soliton mass would be given, instead of $(6.11)$, by

$$
M=4 \pi s R^{2}+\frac{4}{3} \pi p R^{3}+E_{\mathrm{k}},
$$

where $E_{\mathrm{k}}$ is the kinetic energy density (6.7). Because $\partial M / \partial R=0$, we have

$$
8 \pi s R^{2}+4 \pi p R^{3}=E_{\mathrm{k}},
$$

and therefore

$$
M=12 \pi s R^{2}+\frac{16}{3} \pi p R^{3}
$$

Next, we turn on the gravitational field. The critical mass $M_{\mathrm{c}}$ can again be estimated by setting $R \sim 2 G M_{\mathrm{c}}$. This gives (neglecting the factor 2)

$$
1 \sim 12 \pi s G^{2} M_{\mathrm{c}}+\frac{16}{3} \pi p G^{3} M_{\mathrm{c}}^{2},
$$

or

$$
M_{\mathrm{c}} \sim \frac{1}{12 \pi s G^{2}} \frac{2}{1+\sqrt{1+\xi^{2}}}, \quad \text { where } \xi=\frac{2}{3 s}\left(\frac{p}{3 \pi G}\right)^{1 / 2}
$$


Hence, when $p=0$ and $s \neq 0$ we have $\xi=0$ and $M_{c} \sim\left(G^{2} s\right)^{-1}$ as given before by (6.14). If $s=0$ but $p \neq 0$, then $M_{\mathrm{c}} \sim\left(G^{3} p\right)^{-1 / 2}$, which has the same power of $G$ as the Chandrasekhar limit $(6.3)$. Consequently, there is an enormous latitude of $M_{\mathrm{c}}$ for a soliton star, which can vary from a galactic mass to a solar mass.

What happens when $p$ and $s$ are both zero? This, as we shall see, leads to the the mini-boson stars. Consider the simple theory consisting of only a "free" spin 0 complex field $\phi$ of mass $m \neq 0$, plus gravity. Because of the Bose-Einstein statistics, all bosons can be in the same microscopic state of wave length $\sim m^{-1}$; so is then the radius $R$ (even though $N \geqslant 1$ ):

$$
R \sim m^{-1}
$$

Setting $R \sim 2 G M$, one can estimate its critical mass,

$$
M_{\mathrm{c}} \sim\left(l_{\mathrm{P}} m\right)^{-2} m
$$

In the example of $m \sim 30 \mathrm{GeV}, M_{\mathrm{c}} \sim 10^{10} \mathrm{~kg}$, the radius is $\sim 6 \times 10^{-16} \mathrm{~cm}$, and the corresponding density is extremely high, $\sim 10^{+1}$ times that of a neutron star. Because of the smallness of its size, we call such a configuration a mini-boson star.

The details of these exotic stellar configurations will be studied in the following. We start in section 6.2 with the action of the gravitational field from the theory of general relativity, followed by the action of the scalar field in section 6.3. The equations of motion and various mass formulas are given in sections 6.4 and 6.5. In section 6.6, we examine the solutions of the boson stars. The mini-boson stars and their stability are analyzed in section 6.7. The fermion soliton stars are discussed in section 6.8 .

\subsection{Action of gravitational field}

We restrict our investigation to spherically symmetric solutions. The squared length differential can be written in terms of the spherical coordinates $(t, \rho, \alpha, \beta)$ as

$$
\mathrm{d} s^{2}=-\mathrm{e}^{2 u} \mathrm{~d} t^{2}+\mathrm{e}^{2 u} \mathrm{~d} \rho^{2}+\rho^{2}\left(\mathrm{~d} \alpha^{2}+\sin ^{2} \alpha \mathrm{d} \beta^{2}\right) \text {, }
$$

or, in terms of the isotropic coordinates $(t, r, \alpha, \beta)$, as

$$
\mathrm{d} s^{2}=-\mathrm{e}^{2 u} \mathrm{~d} t^{2}+\mathrm{e}^{2 \tilde{v}}\left(\mathrm{~d} r^{2}+r^{2} \mathrm{~d} \alpha^{2}+r^{2} \sin ^{2} \alpha \mathrm{d} \beta^{2}\right) .
$$

where $\alpha$ and $\beta$ are the polar and azimuthal angles, and $\rho$ is $(2 \pi)^{-1}$ times the circumference of a two-sphere, related to $r$ by

$$
\rho=r \mathrm{e}^{\tilde{v}} \text {. }
$$

The functions $u, v$, and $\tilde{v}$ depend on $\rho$ and $t$, or equivalently, on $r$ and $t$. Clearly, $\mathrm{d} s^{2}$ is invariant under the gauge transformation

$$
t \rightarrow t^{\prime}(t), \quad \mathrm{e}^{\prime \prime} \mathrm{d} t \rightarrow \mathrm{e}^{t^{\prime}} \mathrm{d} t^{\prime},
$$


by keeping $\rho, \alpha, \beta$ and $\mathrm{e}^{v}$ fixed. Both of these coordinates are useful in understanding soliton star solutions. We will derive most of the formulae in spherical coordinates.

To write down the gravitational action $A(g)$, it is convenient to consider first a large two-sphere of radius $\rho_{0}$. Let $\mathrm{S}$ be the three-dimensional surface formed by the direct product of the two-sphere

$$
\rho=\rho_{0}
$$

times the time-axis, bounded by

$$
t= \pm T
$$

(both $\rho_{0}$ and $T$ will $\rightarrow \infty$ in the end), and $\Omega$ be the four-dimensional volume within $\mathrm{S}$. We have

$$
A(g)=(16 \pi G)^{-1}\left(\int_{\Omega} \mathscr{R} \mathrm{d} \tau+\int_{\mathrm{S}} \mathscr{S} \mathrm{d} S\right)
$$

where $G$ is Newton's gravitational constant and, according to Einstein's general relativity, $\mathscr{R}$ is the scalar curvature

$$
\begin{aligned}
\mathscr{R}= & 2 \rho^{-2}+2 \mathrm{e}^{-2 u}\left[\frac{\partial^{2} u}{\partial t^{2}}-\frac{\partial v}{\partial t} \frac{\partial u}{\partial t}+\left(\frac{\partial v}{\partial t}\right)^{2}\right] \\
& +2 \mathrm{e}^{-2 v}\left[-\frac{\partial^{2} u}{\partial \rho^{2}}-\left(\frac{\partial v}{\partial \rho}\right)^{2}+\frac{\partial u}{\partial \rho} \frac{\partial v}{\partial \rho}+2 \rho^{-1}\left(\frac{\partial v}{\partial \rho}-\frac{\partial u}{\partial \rho}\right)-\rho^{-2}\right]
\end{aligned}
$$

The derivation of $\mathscr{R}$ in spherical coordinates can be found in most standard textbooks on general relativity. $\mathrm{d} \tau$ is the volume element

$$
\mathrm{d} \tau=|g|^{1 / 2} \mathrm{~d} t \mathrm{~d} \rho \mathrm{d} \alpha \mathrm{d} \beta, \text { with }|g|^{1 / 2}=\mathrm{e}^{u+v} \rho^{2} \sin \alpha
$$

In (6.26), the volume integral part is the usual Hilbert action; the surface term is given by [121]

$$
\mathscr{S}=2 n_{; \mu}^{\mu}+\text { constant }
$$

where $n_{; \mu}^{\mu}$ is the covariant divergence of the (four-dimensional) unit vector $n^{\mu}$ normal to $\mathrm{S}$, and $\mathrm{d} S$ is the surface element on $S$, so that at points on $S$

$$
n_{\mu} \mathrm{d} x^{\mu} \mathrm{d} S=\mathrm{d} \tau
$$

By construction, the invariant character of $A(g)$ under an arbitrary coordinate transformation is manifest. On $\rho=\rho_{0}$, choose the constant in (6.29) to be $-4 / \rho_{0}$; since $n_{\rho}=\mathrm{e}^{v}$ we have

$$
\int \mathscr{S} \mathrm{d} S=8 \pi \int\left\{\rho^{2} \mathrm{e}^{u}\left[\mathrm{e}^{-v}\left(\frac{\partial u}{\partial \rho}+\frac{2}{\rho}\right)-\frac{2}{\rho}\right]\right\}_{\rho=\rho_{0}} \mathrm{~d} t .
$$


On $t=T$, choose the constant in (6.29) to be zero; since $n_{t}=\mathrm{e}^{\prime \prime}$ we have

$$
\int \mathscr{T} \mathrm{d} S=-8 \pi \int\left(\rho^{2} \mathrm{e}^{-u+v} \frac{\partial v}{\partial t}\right)_{t=T} \mathrm{~d} \rho
$$

(likewise, on $t=-T$ ). Therefore, after letting $\rho_{0}$ and $T \rightarrow x,(6.26)$ can be written as

$$
\begin{aligned}
& A(g)=\int \mathrm{d} t L(g), \\
& L(g)=(2 G)^{-1} \int_{0}^{x}\left(\mathrm{e}^{u+v}+\mathrm{e}^{u-v}\left(2 \rho \frac{\partial v}{\partial \rho}-1\right)+\frac{\partial}{\partial \rho}\left[2 \rho\left(\mathrm{e}^{u-v}-\mathrm{e}^{u}\right)\right]\right) \mathrm{d} \rho .
\end{aligned}
$$

The inclusion of the surface integration in (6.26) converts Hilbert's action into one that contains only the first derivative of the metric, so that the usual Lagrangian mechanics formulation can be applicable.

\subsection{Action of scalar field}

For a complex spin- 0 field $\phi$ (with no direct meson-meson coupling) the matter action is

$$
A(m)=-\int\left(\phi^{\star \mu} \phi_{\mu}+m^{2} \phi^{\dagger} \phi\right) \mathrm{d} \tau
$$

where the dagger denotes the Hermitian conjugate.

$$
\phi_{\mu}=\partial \phi / \partial x^{\mu}, \quad \phi_{\mu}^{\ddagger}=\partial \phi^{\dagger} / \partial x^{\mu}, \quad \phi^{\mu}=g^{\mu v} \phi_{v}, \quad \phi^{\dagger \mu}=g^{\mu v} \phi_{,}^{\dagger} .
$$

Both $\phi_{\mu}$ and $\phi_{\mu}^{\dagger}$ are bona fide four-vectors. Writing

$$
A(m)=\int L(m) \mathrm{d} t
$$

we have

$$
L(m)=4 \pi \int_{0}^{x} \mathrm{e}^{u+v} \rho^{2}(-U-V+W) \mathrm{d} \rho .
$$

where

$$
U=U\left(\phi^{\dagger} \phi\right), \quad V=\mathrm{e}^{-2 v} \frac{\partial \phi^{\dagger}}{\partial \rho} \frac{\partial \phi}{\partial \rho}, \quad W=\mathrm{e}^{-2 u} \frac{\partial \phi^{\dagger}}{\partial t} \frac{\partial \phi}{\partial t} .
$$

The Lagrangian of the entire system is

$$
L=L(g)+L(m) \text {. }
$$




\subsection{Equations of motion for boson stars}

Consider a variation $\delta g_{\mu \nu}$ in the metric; the corresponding variations of the actions are

$$
\delta A(g)=-(16 \pi G)^{-1} \int_{\Omega} G^{\mu \nu} \delta g_{\mu \nu} \mathrm{d} \tau, \quad \delta A(m)=-\frac{1}{2} \int_{\Omega} T^{\mu \nu} \delta g_{\mu \nu} \mathrm{d} \tau,
$$

with

$$
G_{\mu \nu}=R_{\mu \nu}-\frac{1}{2} g_{\mu \nu} \mathscr{R}, \quad T_{\mu \nu}=-\phi_{\mu}^{\dagger} \phi_{\nu}-\phi_{\nu}^{\dagger} \phi_{\mu}+g_{\mu \nu}\left[\phi^{\dagger \kappa} \phi_{\kappa}+U\left(\phi^{\dagger} \phi\right)\right],
$$

where $R_{\mu \nu}$ is the Ricci tensor. Since the total action of the system is the sum of $A(g)$ and $A(m)$, we have the Einstein equation

$$
G_{\mu \nu}=-8 \pi G T_{\mu \nu}
$$

For spherically symmetric solutions, the nontrivial equations (in spherical coordinates) are

$$
\begin{aligned}
& G_{t}^{t}=\rho^{-2}\left(\mathrm{e}^{-2 v}-1-2 \mathrm{e}^{-2 v} \rho \partial v / \partial \rho\right)=-8 \pi G(U+V+W) \\
& G_{\rho}^{\rho}=\rho^{-2}\left(\mathrm{e}^{-2 v}-1+2 \mathrm{e}^{-2 v} \rho \partial u / \partial \rho\right)=8 \pi G(-U+V+W) \\
& G_{\alpha}^{\alpha}=G_{\beta}^{\beta}=\mathrm{e}^{-2 u}\left[-\frac{\partial^{2} v}{\partial t^{2}}+\frac{\partial v}{\partial t}\left(\frac{\partial u}{\partial t}-\frac{\partial v}{\partial t}\right)\right]+\mathrm{e}^{-2 v}\left[\frac{\partial^{2} u}{\partial \rho^{2}}+\left(\frac{\partial u}{\partial \rho}+\frac{1}{\rho}\right)\left(\frac{\partial u}{\partial \rho}-\frac{\partial v}{\partial \rho}\right)\right] \\
&=8 \pi G(-U-V+W) \\
& \mathrm{e}^{2 v} G_{t}^{\rho}=-\mathrm{e}^{2 u} G_{\rho}^{t}=2 \rho^{-1} \frac{\partial v}{\partial t}=8 \pi G\left(\frac{\partial \phi^{\dagger}}{\partial t} \frac{\partial \phi}{\partial \rho}+\frac{\partial \phi^{\dagger}}{\partial \rho} \frac{\partial \phi}{\partial t}\right)
\end{aligned}
$$

where

$$
V=\mathrm{e}^{-2 v} \frac{\partial \phi^{\dagger}}{\partial \rho} \frac{\partial \phi}{\partial \rho}, \quad W=\mathrm{e}^{-2 u} \frac{\partial \phi^{\dagger}}{\partial t} \frac{\partial \phi}{\partial t} .
$$

The variation of $\phi$ yields the field equation of the spin- 0 field

$$
\mathrm{e}^{-2 u}\left[-\frac{\partial^{2} \phi}{\partial t^{2}}+\left(\frac{\partial u}{\partial t}-\frac{\partial v}{\partial t}\right) \frac{\partial \phi}{\partial t}\right]+\mathrm{e}^{-2 v}\left[\frac{\partial^{2} \phi}{\partial \rho^{2}}+\left(\frac{\partial u}{\partial \rho}-\frac{\partial v}{\partial \rho}+\frac{2}{\rho}\right) \frac{\partial \phi}{\partial \rho}\right]-\phi \frac{\mathrm{d} U}{\mathrm{~d}(\bar{\phi} \phi)}=0
$$

Equations (6.43) and (6.44) can be derived by setting the variational derivatives of $L$ with respect to $u$ and $v$ to be zero. The result of the equations, (6.45) and (6.46), can be obtained by using the identity

$$
G_{\nu ; \mu}^{\mu}=0
$$

When $\nu=\alpha,(6.49)$ gives $G_{\alpha}^{\alpha}=G_{\beta}^{\beta}$. When $\nu=t$ (and for $\phi$ regular at $\rho=0$ ), it gives (6.46); when $\nu=\rho$, it yields $(6.45)$. 
The matter current $j^{\mu}$, defined by

$$
j^{\mu} \equiv-\mathrm{i}\left(\phi^{\dagger} \phi^{\mu}-\phi^{\dagger \mu} \phi\right)
$$

is conserved: $j_{; \mu}^{\mu}=0$ and therefore

$$
\left(\partial / \partial x^{\mu}\right)\left(|g|^{1 / 2} j^{\mu}\right)=0
$$

This leads to the conservation of the particle number,

$$
N \equiv \int j^{t}|g|^{1 / 2} \mathrm{~d}^{3} x=4 \pi \mathrm{i} \int_{0}^{x} \mathrm{e}^{v-u} \rho^{2}\left(\phi^{\dagger} \frac{\partial \phi}{\partial t}-\frac{\partial \phi^{\dagger}}{\partial t} \phi\right) \mathrm{d} \rho
$$

In section 2.1, we show that at any given particle number $N$, the minimum energy solution $\phi$ has a harmonic dependence on $t$ :

$$
\phi \propto \mathrm{e}^{-\mathrm{i} \omega t}
$$

The same derivation can be generalized to include gravity. For spherically symmetric solutions we may write

$$
\phi=(1 / \sqrt{2}) \sigma(\rho) \mathrm{e}^{-\mathrm{i} \omega t}
$$

The functions $V, W$ and the particle number $N$ become

$$
V=\frac{1}{2} \mathrm{e}^{-2 v}(\mathrm{~d} \sigma / \mathrm{d} \rho)^{2}, \quad W=\frac{1}{2} \omega^{2} \mathrm{e}^{-2 u} \sigma^{2}, \quad N=4 \pi \omega \int_{0}^{x} \mathrm{e}^{v-u} \rho^{2} \sigma^{2} \mathrm{~d} \rho .
$$

The energy momentum tensor $T_{\mu \nu}$ is now time independent, so is the metric $g_{\mu \nu}$. Hence the functions $u$, $v$ and $\tilde{v}$ are functions of $\rho$ only (or of $r$ only). We can relate $v$ to $\tilde{v}$ by

$$
\mathrm{e}^{-v}=\mathrm{d} \ln \rho / \mathrm{d} \ln r=1+r \mathrm{~d} \tilde{v} / \mathrm{d} r
$$

Equations (6.43)-(6.45) give

$$
\begin{aligned}
& \rho^{-2}\left(\mathrm{e}^{-2 v}-1-2 \mathrm{e}^{-2 v} \rho \mathrm{d} v / \mathrm{d} \rho\right)=-8 \pi G(U+V+W) \\
& \rho^{-2}\left(\mathrm{e}^{-2 v}-1+2 \mathrm{e}^{-2 v} \rho \mathrm{d} u / \mathrm{d} \rho\right)=8 \pi G(-U+V+W) \\
& \mathrm{e}^{-2 v}\left[\frac{\mathrm{d}^{2} u}{\mathrm{~d} \rho^{2}}+\left(\frac{\mathrm{d} u}{\mathrm{~d} \rho}+\frac{1}{\rho}\right)\left(\frac{\mathrm{d} u}{\mathrm{~d} \rho}-\frac{\mathrm{d} v}{\mathrm{~d} \rho}\right)\right]=8 \pi G(-U-V+W)
\end{aligned}
$$

Equation (6.46) is now $G_{t}^{p}=G_{p}^{t}=0$ and (6.48) becomes 


$$
\mathrm{e}^{-2 v}\left[\frac{\mathrm{d}^{2} \sigma}{\mathrm{d} \rho^{2}}+\left(\frac{2}{\rho}+\frac{\mathrm{d} u}{\mathrm{~d} \rho}-\frac{\mathrm{d} v}{\mathrm{~d} \rho}\right) \frac{\mathrm{d} \sigma}{\mathrm{d} \rho}\right]+\omega^{2} \mathrm{e}^{-2 u} \sigma-\frac{\mathrm{d} U}{\mathrm{~d} \sigma}=0
$$

In terms of isotropic coordinates, we have

$$
\begin{aligned}
& 2 \frac{\mathrm{d}^{2} \tilde{v}}{\mathrm{~d} r^{2}}+\left(\frac{\mathrm{d} \tilde{v}}{\mathrm{~d} r}\right)^{2}+\frac{4}{r} \frac{\mathrm{d} \tilde{v}}{\mathrm{~d} r}=-8 \pi G \mathrm{e}^{2 \tilde{v}}(W+V+U) \\
& 2 \frac{\mathrm{d} u}{\mathrm{~d} r} \frac{\mathrm{d} \tilde{v}}{\mathrm{~d} r}+\left(\frac{\mathrm{d} \tilde{v}}{\mathrm{~d} r}\right)^{2}+\frac{2}{r}\left(\frac{\mathrm{d} u}{\mathrm{~d} r}+\frac{\mathrm{d} \tilde{v}}{\mathrm{~d} r}\right)=8 \pi G \mathrm{e}^{2 \tilde{v}}(W+V-U) \\
& \frac{\mathrm{d}^{2} u}{\mathrm{~d} r^{2}}+\frac{\mathrm{d}^{2} \tilde{v}}{\mathrm{~d} r^{2}}+\left(\frac{\mathrm{d} u}{\mathrm{~d} r}\right)^{2}+\frac{1}{r}\left(\frac{\mathrm{d} u}{\mathrm{~d} r}+\frac{\mathrm{d} \tilde{v}}{\mathrm{~d} r}\right)=8 \pi G \mathrm{e}^{2 \tilde{v}}(W-V-U) \\
& \mathrm{e}^{-2 \tilde{v}}\left[\frac{\mathrm{d}^{2} \sigma}{\mathrm{d} r^{2}}+\left(\frac{2}{r}+\frac{\mathrm{d} u}{\mathrm{~d} r}+\frac{\mathrm{d} \tilde{v}}{\mathrm{~d} r}\right) \frac{\mathrm{d} \sigma}{\mathrm{d} r}\right]+\omega^{2} \mathrm{e}^{-2 u} \sigma-\frac{\mathrm{d} U}{\mathrm{~d} \sigma}=0
\end{aligned}
$$

It is useful to introduce

$$
\begin{aligned}
& x \equiv \mathrm{e}^{v} \rho \mathrm{d} u / \mathrm{d} \rho=r \mathrm{~d} u / \mathrm{d} r, \quad y \equiv \mathrm{e}^{-v}=1+r \mathrm{~d} \tilde{v} / \mathrm{d} r \\
& \dot{x} \equiv \mathrm{e}^{v} \rho \mathrm{d} x / \mathrm{d} \rho, \quad \dot{y} \equiv \mathrm{e}^{v} \rho \mathrm{d} y / \mathrm{d} \rho .
\end{aligned}
$$

We can write $(6.61)$

$$
\begin{aligned}
& 2 \dot{y}+y^{2}-1=-8 \pi G \rho^{2}(W+V+U), \\
& 2 x y+y^{2}-1=8 \pi G \rho^{2}(W+V+U), \\
& \dot{x}+\dot{y}+x^{2}=8 \pi G \rho^{2}(W-V-U) .
\end{aligned}
$$

These three equations are particularly useful in the regions where one of $W, V$, or $U$ dominates.

\subsection{Hamiltonian and mass formulae} $\mathcal{V}$

Write the Lagrangian (6.40) as the difference between the kinetic energy $\mathscr{K}$ and the potential energy

$$
L=\mathscr{K}-\mathscr{V},
$$

where

$$
\mathscr{K}=4 \pi \int_{0}^{\infty} \mathrm{e}^{u+v} \rho^{2} W \mathrm{~d} \rho=4 \pi \int_{0}^{\infty} \mathrm{e}^{-u+v} \rho^{2} \frac{\partial \phi^{\dagger}}{\partial t} \frac{\partial \phi}{\partial t} \mathrm{~d} \rho,
$$


and $\mathscr{V}$ is the sum of a matter component $\mathscr{V}(m)$ and a gravitational $\mathscr{V}(g)$ component

$$
\mathscr{V}=\mathscr{V}(m)+\mathscr{V}(g)
$$

On account of $(6.38)$,

$$
\mathscr{V}(m)=4 \pi \int_{0}^{x} \mathrm{e}^{u+v} \rho^{2}(U+V) \mathrm{d} \rho
$$

and from (6.34)

$$
\mathscr{V}(g)=-L(g)=(2 G)^{-1} \int_{0}^{x} \mathrm{e}^{u}\left[-\mathrm{e}^{v}+2(1+\rho \partial u / \partial \rho)-\mathrm{e}^{-v}(1+2 \rho \partial v / \partial \rho)\right] \mathrm{d} \rho .
$$

Our spherically symmetric configuration eliminates the kinetic energy carried by the gravitational field. Throughout the paper, we assume that $u$ and $v$ are regular at $\rho=0$. As we shall see, when $\rho \rightarrow \infty$, the matter density goes to zero exponentially; hence from (6.57) it follows that $v$ is $\mathrm{O}\left(\rho^{-1}\right)$ at $\rho=\infty$. Define

$$
\lim _{\rho \rightarrow \infty} \rho v \equiv G M .
$$

From (6.58) we find the same limit for $-u$, i.e.

$$
\lim _{\rho \rightarrow \infty} \rho u=-G M
$$

with the same constant $M$, in agreement with the Schwarzschild solution.

The usual canonical formalism can be readily carried out. The conjugate momenta of $\phi$ and $\phi^{\dagger}$ are

$$
\Pi=4 \pi \rho^{2} \mathrm{e}^{-u+v} \partial \phi^{\dagger} / \partial t, \quad \Pi^{\dagger}=4 \pi \rho^{2} \mathrm{e}^{-u+v} \partial \phi / \partial t
$$

The Hamiltonian $H\left(u, v, \phi, \phi^{\dagger}, \Pi, \Pi^{\dagger}\right)$ is

$$
H=\mathscr{K}+\mathscr{V}
$$

where the same $\mathscr{K}(6.68)$ is now expressed as

$$
\mathscr{K}=\int_{0}^{x}\left(4 \pi \rho^{2}\right)^{-1} \mathrm{e}^{u-v} \Pi^{\dagger} \Pi \mathrm{d} \rho
$$

Hamilton's equations for $\phi, \phi^{\dagger}, \Pi$ and $\Pi^{\dagger}$ are

$$
\frac{\partial \phi}{\partial t}=\frac{\delta H}{\delta \Pi}, \quad \frac{\partial \phi^{\dagger}}{\partial t}=\frac{\delta H}{\delta \Pi^{\dagger}}, \quad \frac{\partial \Pi}{\partial t}=-\frac{\delta H}{\delta \phi}, \quad \frac{\partial \Pi^{\dagger}}{\partial t}=-\frac{\delta H}{\delta \phi^{\dagger}}
$$

they give the matter equation (6.60). Because $L$ does not contain any time-derivatives of $u$ and $v$, the 
conjugate momenta of $u$ and $v$ are both zero. Thus, Hamilton's equations for $u$ and $v$ are simply

$$
\frac{\delta H}{\delta u}=0, \quad \frac{\delta H}{\delta v}=0 .
$$

Since $u$ is regular at $\rho=0$ and $\mathrm{O}\left(\rho^{-1}\right)$ at $\rho=\infty$, the same holds for $\delta u$. From (6.69), we find

$$
\begin{aligned}
\delta \mathscr{V}(g)= & (2 g)^{-1} \int_{0}^{\infty} \mathrm{e}^{u+v}\left\{\left[-1+\mathrm{e}^{-2 v}(1-2 \rho \partial v / \partial \rho)\right] \delta u\right. \\
& \left.+\left[-1+\mathrm{e}^{-2 v}(1+2 \rho \partial u / \partial \rho)\right] \delta v\right\} \mathrm{d} \rho
\end{aligned}
$$

which makes Hamilton's equations (6.78) the same as Lagrange's equations (6.43)-(6.45).

Substituting the solution of $\delta H / \delta u=0$ into the Hamiltonian (6.75), we obtain

$$
H=\lim _{\rho \rightarrow \infty} G^{-1} \rho\left(\mathrm{e}^{u}-\mathrm{e}^{u-v}\right)=M,
$$

in agreement with the ADM [122] definition of mass. Noting that the left-hand side of $(6.57)$ is

$$
\rho^{-2} \mathrm{~d}\left[\rho\left(\mathrm{e}^{-2 v}-1\right)\right] / \mathrm{d} \rho,
$$

the same $M$ is also given by the positive expression

$$
M=4 \pi \int_{0}^{\infty}(U+V+W) \rho^{2} \mathrm{~d} \rho .
$$

By taking the combination $G_{\alpha}^{\alpha}+\frac{1}{2}\left(G_{\rho}^{\rho}-G_{t}^{t}\right)$, we have

$$
\frac{\mathrm{d}}{\mathrm{d} \rho}\left(\rho^{2} \mathrm{e}^{u-v} \frac{\mathrm{d} u}{\mathrm{~d} \rho}\right)=8 \pi G \rho^{2} \mathrm{e}^{u+v}(2 W-U)
$$

which leads to still another formula for $M$,

$$
M=8 \pi \int_{0}^{\infty}(2 W-U) \mathrm{e}^{u+v} \rho^{2} \mathrm{~d} \rho .
$$

\subsection{Boson stars}

The simplest boson star solution [26] is for $U$ given by

$$
U=m^{2} \phi^{\dagger} \phi\left[1-\left(2 \phi^{\dagger} \phi / \sigma_{0}^{2}\right)^{2}\right]^{2},
$$

as before, where $\phi^{\dagger}$ is the Hermitian conjugate of $\phi$, and $\sigma_{0}$ and $m$ are constants. There are two degenerate minima of $U$. We call $|\phi|=0$ the normal vacuum, which is maintained at infinity. In the 
normal vacuum $m$ is the mass of the plane-wave solution. From (6.54) we have

$$
U=\frac{1}{2} m^{2} \sigma^{2}\left[1-\left(\sigma / \sigma_{0}\right)^{2}\right]^{2} .
$$

For a very large particle number $N$, we know from section 2.2 that nontopological soliton solutions exist in the absence of gravity. To the first approximation, the soliton is a sphere of radius $R$ : Inside the sphere, $|\phi|$ is a constant equal to $\sigma_{0}$. At the surface of the sphere $|\phi|$ sharply drops to zero within a range of order of $1 / \mathrm{m}$. Outside the sphere $\phi=0$. One may take a trial function

$$
\sigma= \begin{cases}\sigma_{0}, & \rho<R+\mathrm{O}\left(m^{-1}\right), \\ 0, & \rho>R+\mathrm{O}\left(m^{-1}\right),\end{cases}
$$

and, when $\rho=R+\mathrm{O}\left(m^{-1}\right)$,

$$
\sigma \simeq \sigma_{0} /\left(1+\mathrm{e}^{2 m(\rho-R)}\right)^{1 / 2} .
$$

The above expression (6.88) is chosen because it satisfies

$$
\mathrm{d}^{2} \sigma / \mathrm{d} \rho^{2}-\mathrm{d} U / \mathrm{d} \sigma=0
$$

and the boundary condition $\sigma=0$ at infinity. Using this trial function, one finds that the energy of the soliton is given approximately by

$$
E=E_{\mathrm{k}}+E_{\mathrm{s}},
$$

where $E_{\mathrm{k}}$ is the kinetic energy due to the interior $\rho<R$,

$$
E_{\mathrm{k}} \simeq\left(\frac{4}{3} \pi R^{3}\right) \frac{1}{2} \omega^{2} \sigma_{0}^{2},
$$

and $E_{\mathrm{s}}$ is the surface energy due to the transition region $\rho=R+\mathrm{O}\left(\mathrm{m}^{-1}\right)$,

$$
E_{\mathrm{s}} \simeq 4 \pi s R^{2} \text {, where } s=\frac{1}{4} m \sigma^{2} .
$$

The frequency $\omega$ is related to the particle number $N$ by

$$
N \simeq \frac{4}{3} \pi R^{3} \omega \sigma_{0}^{2} .
$$

Keeping $N$ fixed, and setting $\partial E / \partial R=0$ and $M=E_{\mathrm{k}}=E_{\mathrm{s}}$ we see that

$$
\begin{aligned}
& E_{\mathrm{k}}=\frac{2}{3} E_{\mathrm{s}}, \\
& M=\frac{5}{3} E_{\mathrm{s}}=5 \times 4 \pi s R^{2} .
\end{aligned}
$$

Hence

$$
M \propto N^{4 / 5}, \quad R \propto N^{2 / 5} \propto M^{1 / 2}, \quad \omega N^{-1 / 5} \propto M^{-1 / 4} .
$$


As the soliton mass $M$ increases, it is clear that the gravity becomes important only if $R$ is of the order of the Schwarzschild radius $2 G M$. From (6.95), we obtain an estimate of the critical mass, as in (6.14)

$$
M_{\mathrm{c}} \sim\left(G^{2} s\right)^{-1} \sim\left(l_{\mathrm{P}}^{4} m \sigma_{0}\right)^{-1}
$$

Using equations (6.87) and (6.88) and treating $\sigma_{0} \sim m \ll l_{\mathrm{P}}^{-1}, M \sim M_{\mathrm{c}}$, we can estimate the relative importance of $U, V$ and $W$ introduced in (6.39). Because $V$ and $W$ are proportional to $(\mathrm{d} \sigma / \mathrm{d} \rho)^{2}$ and $\omega^{2} \sigma^{2}$, in the interior $\rho<R+\mathrm{O}\left(m^{-1}\right)$ we expect

$$
V / W \sim 1 / \omega^{2} R^{2} \sim(m / M)^{1 / 2} \ll 1
$$

Neglecting $\mathrm{d} \sigma / \mathrm{d} \rho$ in the field equation of $\sigma(6.60)$, we find

$$
1-\left(\sigma / \sigma_{0}\right)^{2} \simeq-\frac{1}{2}\left(\omega^{2} / m^{2}\right) \mathrm{e}^{-2 u},
$$

and therefore

$$
U / W \sim \omega^{2} / m^{2} \sim(m / M)^{1 / 2} \ll 1
$$

For $\sigma_{0}=\mathrm{O}(m)$, the parameter

$$
\lambda \equiv(8 \pi G)^{1 / 2} \sigma_{0}
$$

is very small, e.g., for $\sigma_{0} \sim 100 \mathrm{GeV}, \lambda \sim 10^{-16}$. It is straightforward to verify that

$$
R \sim G M \sim\left(\lambda^{2} m\right)^{-1}, \quad \omega / m \sim \lambda .
$$

If we define

$$
\bar{\rho} \equiv \lambda^{2} m \rho, \quad \mathrm{e}^{-\bar{u}} \equiv(\omega / \lambda m) \mathrm{e}^{-u}, \quad \bar{v}=v,
$$

then $\bar{\rho} \sim 1$. Keeping only the lowest order in $\lambda$, the equation for $\sigma(6.60)$, in the interior region becomes

$$
\omega^{2} \mathrm{e}^{-2 u} \sigma-\mathrm{d} U / \mathrm{d} \sigma=\mathrm{O}\left(\lambda^{4}\right) .
$$

This leads to

$$
\sigma / \sigma_{0}=1+\frac{1}{4} \lambda^{2} \mathrm{e}^{-2 \bar{u}}+\mathrm{O}\left(\lambda^{4}\right) .
$$

The Einstein equations (6.57)-(6.60) yield a set of two equations independent of matter field,

$$
\begin{aligned}
& 2 \bar{\rho} \mathrm{d} \bar{v} / \mathrm{d} \bar{\rho}=\left(\frac{1}{2} \mathrm{e}^{-2 \bar{u}} \bar{\rho}^{2}-1\right) \mathrm{e}^{2 \bar{v}}+1, \\
& 2 \bar{\rho} \mathrm{d} \bar{u} / \mathrm{d} \bar{\rho}=\left(\frac{1}{2} \mathrm{e}^{-2 \bar{u}} \bar{\rho}^{2}+1\right) \mathrm{e}^{2 \bar{v}}-1,
\end{aligned}
$$


because

$$
W \pm V \pm U=W\left[1+\mathrm{O}\left(\lambda^{2}\right)\right]
$$

where $\bar{v} \equiv v$. When $\bar{\rho} \rightarrow 0$, these equations determine

$$
\begin{aligned}
& \bar{u}=\bar{u}(0)+\frac{1}{6} \mathrm{e}^{-2 \bar{u}(0)} \bar{\rho}^{2}+\mathrm{O}\left(\bar{\rho}^{-4}\right), \\
& \bar{v}=\frac{1}{12} \mathrm{e}^{-2 \bar{u}(0)} \bar{\rho}^{-2}+\mathrm{O}\left(\bar{\rho}^{-4}\right) .
\end{aligned}
$$

So the interior solution can be obtained by first assigning at $\bar{\rho}=0$ an initial value

$$
\mathrm{e}^{-\bar{u}(0)}=(\omega / \lambda m) \mathrm{e}^{-u(0)}
$$

and then integrating (6.106) and (6.107) numerically from $\rho=0$ to $R$, i.e. from $\bar{\rho}=0$ to $\bar{\rho}=\lambda^{2} m R$. Actually one only needs to integrate these coupled equations (6.106) and (6.107) once for an arbitrary value of $\vec{u}(0)$, say $\bar{u}(0)=0$, from $\bar{\rho}=0$ to $\bar{\rho}=\infty$; the solutions for other initial values can be obtained by a simple transformation

$$
\mathrm{e}^{-\bar{u}} \rightarrow \kappa \mathrm{e}^{-\bar{u}}, \quad \bar{\rho} \rightarrow \bar{\rho} / \kappa, \quad \bar{v} \rightarrow \bar{v} .
$$

Equations (6.106) and (6.107) are invariant under the transformation (6.113). One way of observing this invariance is to look at the equations for $x$ and $y,(6.64)-(6.66)$. The quantities $x, y, \dot{x}$ and $\dot{y}$ are all invariant under the transformation. So are then the left-hand sides of (6.64)-(6.66). On the right-hand sides because of (6.108), only the $W$ term survives. Eliminating $W$ from eqs. (6.64)-(6.66), we have

$$
\dot{y}=1-x y-y^{2}, \quad \dot{x}=-2-x^{2}+3 x y+2 y^{2} .
$$

Therefore,

$$
\frac{\mathrm{d} y}{\mathrm{~d} x}=\frac{1-x y-y^{2}}{-2-x^{2}+3 x y+2 y^{2}} .
$$

It is convenient to introduce $\tau$ as a fictitious "time", where

$$
\mathrm{d} / \mathrm{d} \tau=\mathrm{e}^{v} \rho \mathrm{d} / \mathrm{d} \rho
$$

As $\tau$ varies, each solution describes a trajectory in the $(x, y)$ plane. On account of $(6.105)$ and $(6.111)$, when $\bar{\rho} \rightarrow 0$ (therefore $\tau \rightarrow \infty$ ),

$$
x=\frac{1}{3} \mathrm{e}^{-2 \bar{u}(0)} \bar{\rho}^{-2}+\mathrm{O}\left(\bar{\rho}^{-4}\right), \quad y=1-\frac{1}{12} \mathrm{e}^{-2 \bar{u}(0)} \bar{\rho}^{2}+\mathrm{O}\left(\bar{\rho}^{-4}\right) .
$$

There are two critical points of $(6.114)$, defined by $\dot{x}=\dot{y}=0$ :

(i) $x=0, y=1 ;$ (ii) $x=y=1 / \sqrt{2}$. 
From (6.116), we see that at $\rho=0$ the trajectory begins at (i) with an initial slope

$$
[\mathrm{d} y / \mathrm{d} x]_{0}=-\frac{1}{4}
$$

When $\rho$ increases from 0 to $R$, the interior solution moves along a universal trajectory, called $I$ (the interior), in the $(x, y)$ plane; trajectory $\mathrm{I}$ is completely determined by the first-order differential equation (6.114) with the initial condition (6.118), and is shown by the solid curve in fig. 18 .

At $\rho=R_{-}$, the solution is at a point, called "in" (denoting the inner face of the surface) on I, with

$$
x=x_{\text {in }}, \quad y=y_{\text {in }} .
$$

As shown in fig. 19 (and as we shall also prove), in the surface region when $\rho$ decreases from $R_{-}$to $R_{+}$,

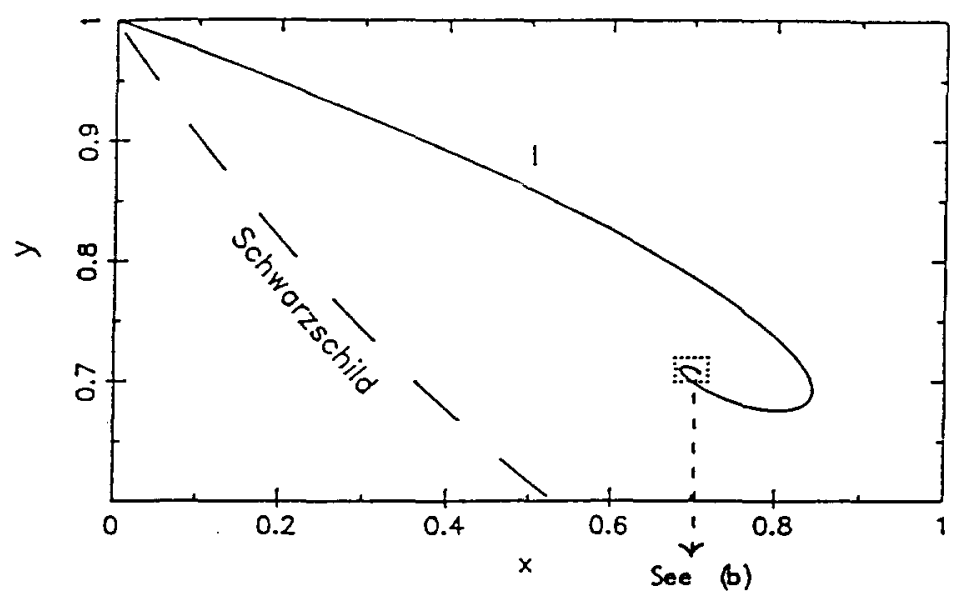

(a)

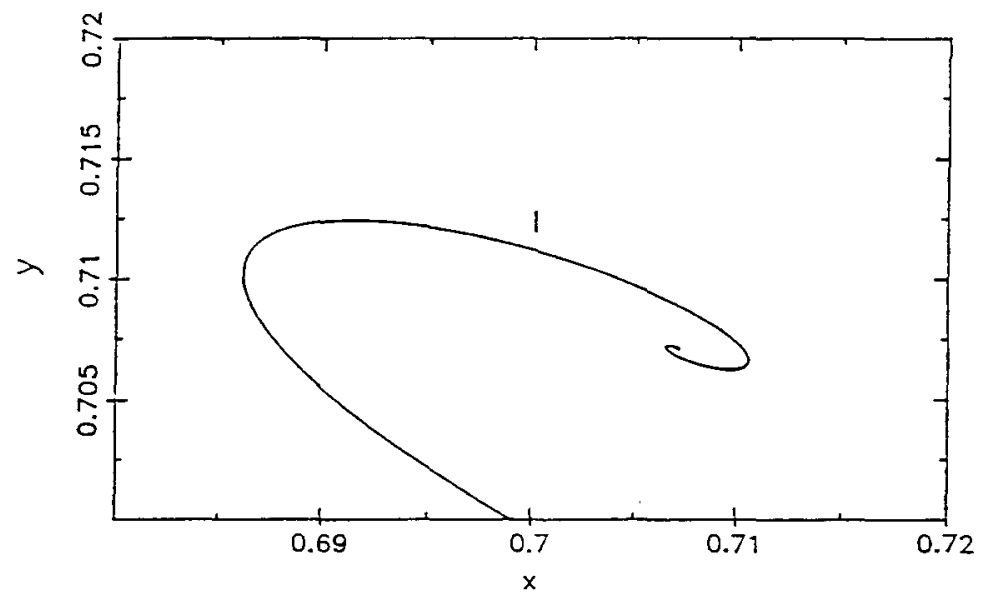

(b)

Fig. 18. The solid curve is the universal ( $W$-dominated) trajectory. The dashed curve is the Schwarzschild hyperbola $2 x y+y^{2}-1=0$. 

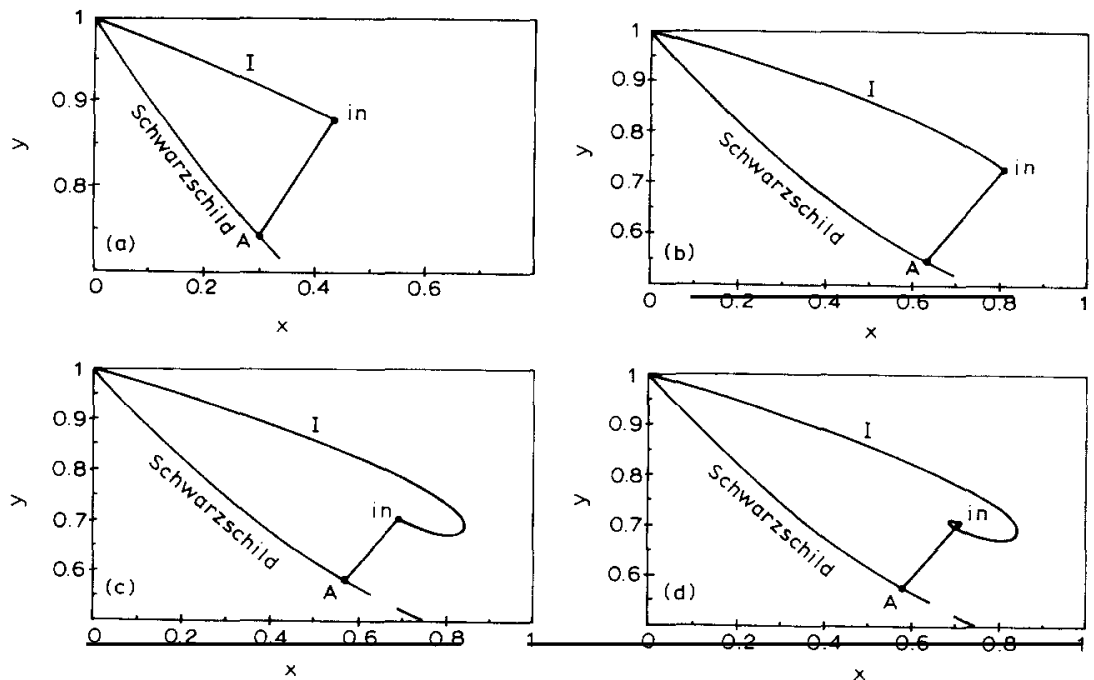

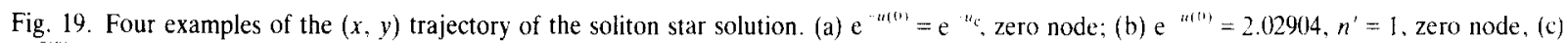
$\mathrm{e}^{-\bar{u}(0)}=16.5784, n^{\prime}=2$, zero node; (d) $\mathrm{e}^{-\bar{x}(0)}=-96.9098, n^{\prime}=3$, zero node.

the solution leaves I abruptly; it moves along the straight line

$$
x-x_{\text {in }}=y-y_{\text {in }},
$$

and ends at a point, called

$$
\mathrm{A}:\left(x_{\mathrm{A}}, y_{\mathrm{A}}\right)
$$

on the Schwarzschild hyperbola

$$
2 x y+y^{2}-1=0
$$

with $x_{\mathrm{A}}$ and $y_{\mathrm{A}}$ both $>0$. Afterwards, we are in the exterior region $\rho>R$, which is described by the Schwarzschild solution. The trajectory then moves along the hyperbola (6.121) from A when $\rho=R_{+}$, back to the point (i) when $\rho=\infty$. Different solutions are characterized only by different points $\left(x_{\text {in }}, y_{\text {in }}\right)$ when the transition occurs; neither the trajectory I nor the Schwarzschild hyperbola depends on different features of each individual solution.

In the surface region it is more convenient to use the isotropic coordinates, given by $(6.21)$. When $\rho=R$, denote

$$
r=r_{\mathrm{s}}=R \mathrm{e}^{-\tilde{v}_{\mathrm{s}}}, \quad u=u_{\mathrm{s}}, \quad \tilde{v}=\tilde{v}_{\mathrm{s}} .
$$

Within the surface, $\mathrm{d} \sigma / \mathrm{d} r$ is $\mathrm{O}\left(m \sigma_{0}\right)$, but because $x=r \mathrm{~d} u / \mathrm{d} r$ and $y=1+r \mathrm{~d} \tilde{v} / \mathrm{d} r$ are $\sim 1, \mathrm{~d} u / \mathrm{d} r$ and $\mathrm{d} \tilde{v} / \mathrm{d} r$ are both $\mathrm{O}\left(r_{\mathrm{s}}^{-1}\right)$, i.e. $\lambda^{2} \mathrm{O}(m)$. Hence, neglecting $\mathrm{O}\left(\lambda^{2}\right)$, we can regard $u=u_{\mathrm{s}}$ and $\tilde{v}=\tilde{v}_{\mathrm{s}}$ as constants across the surface; in addition, since $\omega^{2} / m^{2}$ is also $\mathrm{O}\left(\lambda^{2}\right),(6.42)$ reduces to

$$
\mathrm{e}^{-2 \tilde{v}_{\mathrm{s}}} \mathrm{d}^{2} \sigma / \mathrm{d} r^{2}-\mathrm{d} U / \mathrm{d} \sigma=0
$$


This gives the solution, valid for $r=r_{\mathrm{s}}+\mathrm{O}\left(m^{-1}\right)$,

$$
\sigma \simeq \sigma_{0} /\left(1+\mathrm{e}^{\mu\left(r-r_{\mathrm{s}}\right)}\right)^{1 / 2}, \text { where } \mu=2 m \mathrm{e}^{\tilde{v}_{\mathrm{s}}} .
$$

From (6.47) and (6.123), to the same accuracy, we have within the surface

$$
U=V=\mathrm{O}\left(m^{2} \sigma_{0}^{2}\right)
$$

but

$$
W=\mathrm{O}\left(\omega^{2} \sigma_{0}^{2}\right)=\lambda^{2} \mathrm{O}(U)
$$

By using solution (6.124), we find the integrals of $U$ and $V$ across the surface to be

$$
\int_{\text {surface }} U \mathrm{~d} r=\int_{\text {surface }} V \mathrm{~d} r=\frac{1}{8} m \sigma_{0}^{2} \mathrm{e}^{-\tilde{v}_{\mathrm{s}}} .
$$

Hence, in the approximation $\lambda^{2}=0$, we may write

$$
U=V=\frac{1}{8} m \sigma_{0}^{2} \mathrm{e}^{-\tilde{v}_{\mathrm{s}}} \delta\left(r-r_{\mathrm{s}}\right), \quad W=0 .
$$

These lead to, in the surface region,

$$
\mathrm{d} x / \mathrm{d} r=\mathrm{d} y / \mathrm{d} r=-\pi G r_{\mathrm{s}} \mathrm{e}^{\tilde{u}_{\mathrm{s}}} m \sigma_{0}^{2} \delta\left(r-r_{\mathrm{s}}\right) .
$$

In the exterior region $r>r_{\mathrm{s}}$, the same approximation $\lambda^{2}=0$ leads to zero matter density, and therefore

$$
U=V=W=0 .
$$

The solution has to lie on the Schwarzschild hyperbola (6.121). Integrating (6.64) and (6.66) across the surface, we see that the discontinuities in $x$ and $y$ from "in" to A are

$$
x_{\text {in }}-x_{\mathrm{A}}=y_{\text {in }}-y_{\mathrm{A}}=\frac{1}{8} \bar{\rho}_{\text {in }} \text {. }
$$

Because $x_{\mathrm{A}}$ and $y_{\mathrm{A}}$ are on the Schwarzschild hyperbola, it follows that

$$
2 x_{\mathrm{A}} y_{\mathrm{A}}+y_{\mathrm{A}}^{2}-1=0 \text {. }
$$

Expressing $x_{\mathrm{A}}$ and $y_{\mathrm{A}}$ in terms of $x_{\text {in }}, y_{\text {in }}$, and $\bar{\rho}_{\text {in }}$, we have

$$
\frac{1}{8} \bar{\rho}_{\mathrm{in}}=\frac{1}{3}\left\{x_{\mathrm{in}}+2 y_{\mathrm{in}}-\left[\left(x_{\mathrm{in}}-y_{\mathrm{in}}\right)^{2}+3\right]^{1 / 2}\right\} \equiv \Delta\left(x_{\mathrm{in}}, y_{\mathrm{in}}\right) \text {. }
$$

Define $\hat{u}(\hat{\rho})$, and $\hat{v}(\hat{\rho})$ to be the solutions of

$$
2 \hat{\rho} \mathrm{d} \hat{v} / \mathrm{d} \hat{\rho}=\left(\frac{1}{2} \mathrm{e}^{-2 \hat{u}} \hat{\rho}^{2}-1\right) \mathrm{e}^{2 \hat{v}}+1, \quad 2 \hat{\rho} \mathrm{d} \hat{u} / \mathrm{d} \hat{\rho}=\left(\frac{1}{2} \mathrm{e}^{-2 \hat{u}} \hat{\rho}^{2}+1\right) \mathrm{e}^{2 \hat{v}}-1,
$$


with the boundary condition

$$
\hat{u}(0)=\hat{v}(0)=0 \quad \text { at } \quad \hat{\rho}=0
$$

Any solution of $(6.104)-(6.107)$ with boundary condition $\bar{u}(0) \neq 0$ and $\bar{v}(0) \neq 0$ can then be derived from $\hat{u}(\hat{\rho})$ and $\hat{v}(\hat{\rho})$ through

$$
\mathrm{e}^{-\bar{u}(\bar{\rho})}=\mathrm{e}^{-\hat{u}(\hat{\rho})-\bar{u}(0)}, \quad \mathrm{e}^{-\bar{v}(\bar{\rho})}=\mathrm{e}^{-\hat{v}(\hat{\rho})}, \quad \text { where } \bar{\rho}=\hat{\rho} \mathrm{e}^{\bar{u}(0)} .
$$

The functions $\hat{u}(\hat{\rho})$ and $\hat{v}(\hat{\rho})$ are plotted in fig. 20 .

By using (6.107) and (6.136), we find

$$
y=\mathrm{e}^{-\hat{v}}, \quad x=y \hat{\rho} \mathrm{d} \hat{u} / \mathrm{d} \hat{\rho} .
$$

Thus, from $\hat{u}(\hat{\rho})$ and $\hat{v}(\hat{\rho})$, we also derive $\hat{x}(\hat{\rho})$ and $\hat{y}(\hat{\rho})$. Equation (6.133) is satisfied by setting

$$
\frac{1}{8} \bar{\rho}=\Delta(x(\hat{\rho}), y(\hat{\rho})) \equiv \Delta(\hat{\rho})
$$

Identifying $\bar{\rho}=\bar{\rho}_{\text {in }}$ and $\hat{\rho}=\hat{\rho}_{\text {in }}$, we have $\mathrm{e}^{i(t)}=\bar{\rho}_{\text {in }} / \hat{\rho}_{\text {in }}$ and therefore all other characteristics of a soliton star.

In fig. 21 the solid curve is $\Delta(\hat{\rho})$ which is independent of $\mathrm{e}^{-u(0)}$, and the dashed line is $\mathrm{e}^{\bar{u}(0)} \hat{\rho} / 8$. For $\mathrm{e}^{-\bar{u}(0)}=2.5$, there are two solutions of (6.138). It is clear that if we decrease $\mathrm{e}^{-\bar{u}(0)}$, the dashed line will swing counterclockwise until it reaches a critical point, called $\mathrm{c}$, when

$$
\mathrm{e}^{-\bar{u}(0)}=\mathrm{e}^{-\bar{u}_{\mathrm{c}}}=1.266
$$

At c, (6.139) has only one unique solution. The corresponding trajectory is given in fig. 19a.
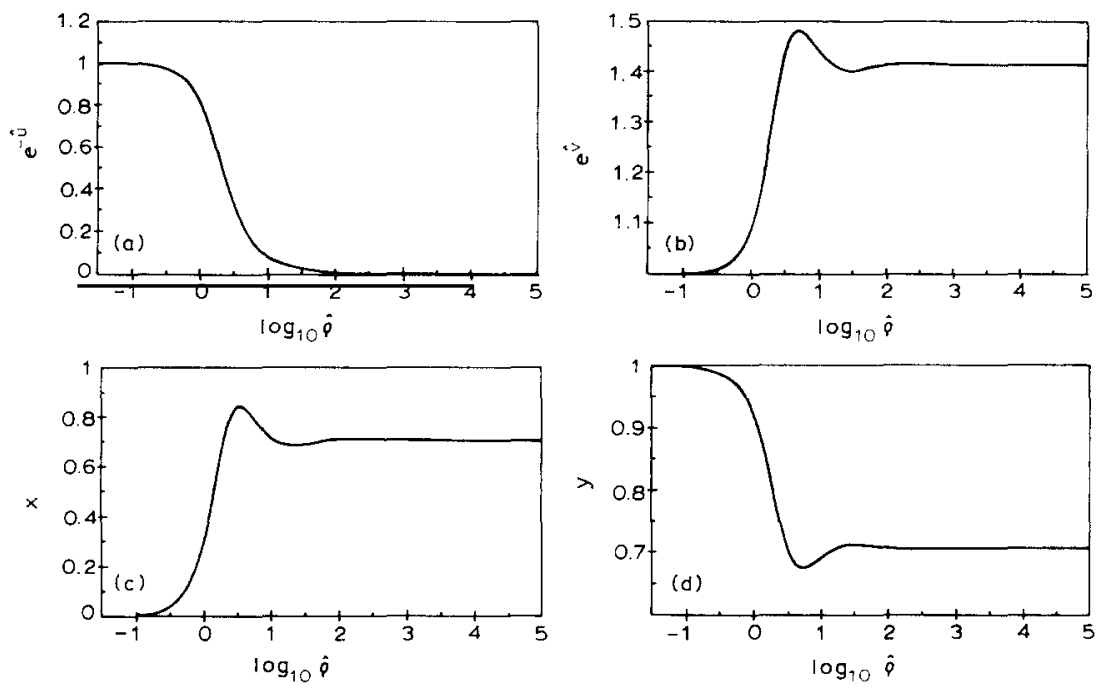

Fig. 20. From solutions of $\hat{u}(\hat{\rho}), \hat{v}(\hat{\rho}), x(\hat{\rho})$, and $y(\hat{\rho})$, one can derive $\vec{u}(\bar{\rho}), \bar{v}(\bar{\rho}), x(\bar{\rho})$ and $y(\bar{\rho})$ for any initial value $\bar{u}(0)$. 


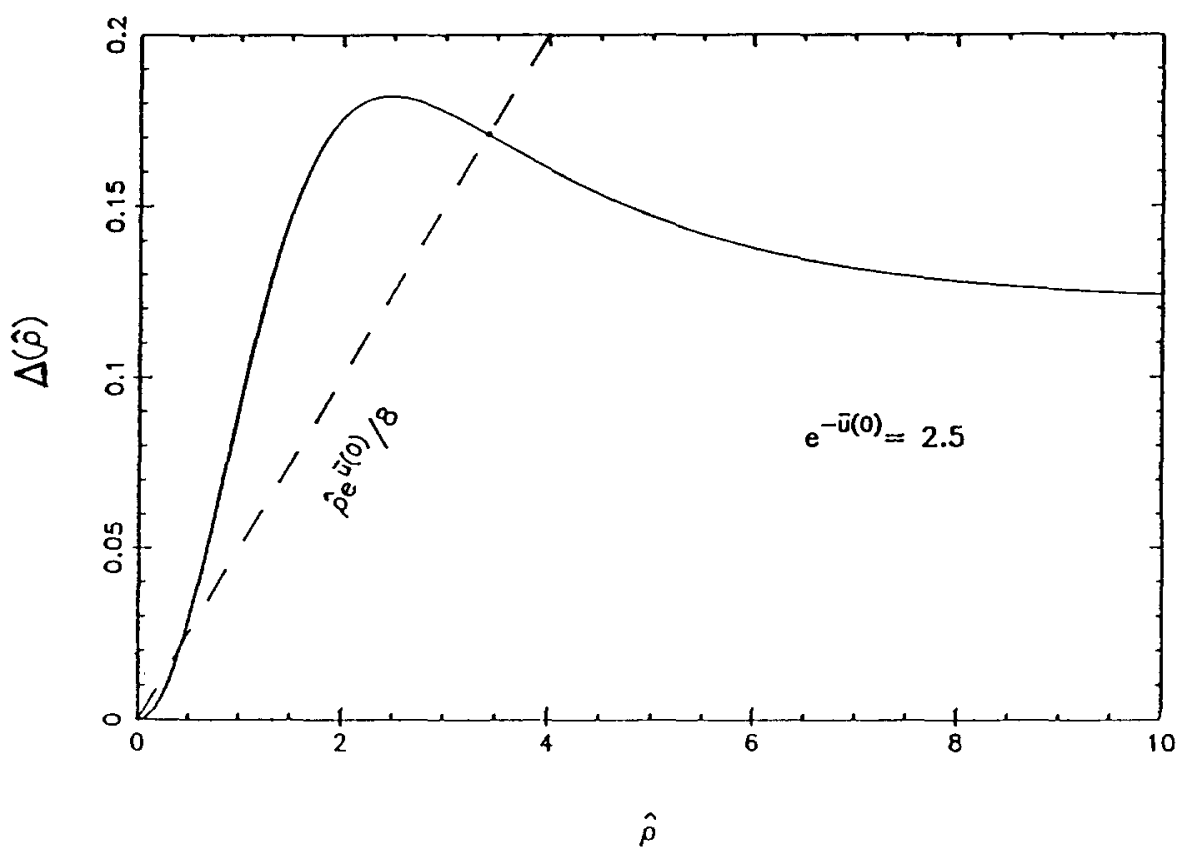

Fig. 21. The initial value $\mathrm{e}^{-\bar{u}(0)}$ determines the slope of the dashed line, $\frac{1}{8} \mathrm{e}^{\bar{u}(0)} \hat{\rho}$, whose intersection with $\Delta(\hat{\rho})$ gives a solution of the soliton star.

For $\mathrm{e}^{-\bar{u}(0)}<\mathrm{e}^{-\bar{u}_{\mathrm{c}}}$, there is no solution. For $\mathrm{e}^{-\bar{u}(0)}>\mathrm{e}^{-\bar{u}_{\mathrm{c}}}$ there are two solutions. By systematically changing $\mathrm{e}^{-\bar{u}(0)}$, we can survey all the zero-node solutions $(n=0)$. In figs. $22 \mathrm{a}$ and $22 \mathrm{~b}, M$ is plotted versus $N$, schematically in $22 \mathrm{a}$ and precisely in 22b. In fig. 23 we show the curves of $M, N$ and $R$ versus $\omega$ and $R$ versus $M$, and in fig. 24, the dependence of $\mathrm{e}^{\bar{u}(0)}, M, \omega$, and $R$ on $\hat{\rho}_{\text {in }}$.

In section 3 , we have shown that cusps on the $M$ versus $N$ curve are closely related to the stability of soliton solutions. If one soliton solution on the lowest branch of the $M$ versus $N$ curve is stable under small perturbations, then the whole lowest branch is stable under small perturbations. When $N$ is small, the effect of gravity is negligible and we know from previous sections that the soliton is stable. Thus all boson star solutions on the lowest branch are stable under small perturbations.

In the above example the surface energy dominates; as a result we have a very large upper limit for the soliton mass. If one chooses a different form for $U(\sigma)$, then the maximum mass for a boson star can be much smaller. In particular, as mentioned in section 6.1, if $p$ dominates in (6.16), or if $U=$ $\frac{1}{2} m^{2} \sigma^{2}+\lambda \sigma^{4}$ with $\lambda>0$ (therefore only one minimum), then the maximum boson star mass becomes of the order of the solar mass, or smaller $[24,123]$.

\subsection{Mini-boson starts $[7,8,25,28]$}

When there is no self-interaction of the scalar field, i.e.

$$
U=\frac{1}{2} m^{2} \sigma^{2},
$$

the nonlinear and attractive nature of the gravitational interaction still allows the existence of another 


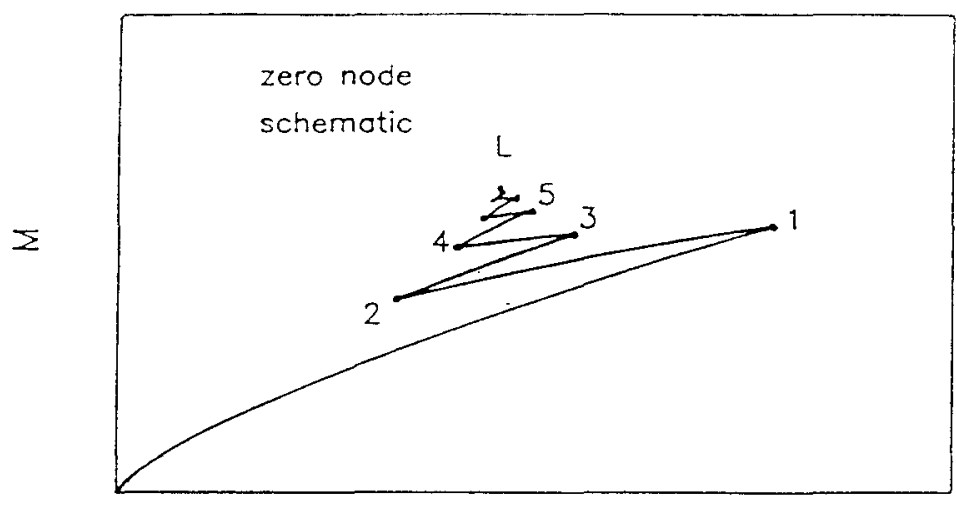

N

(a)

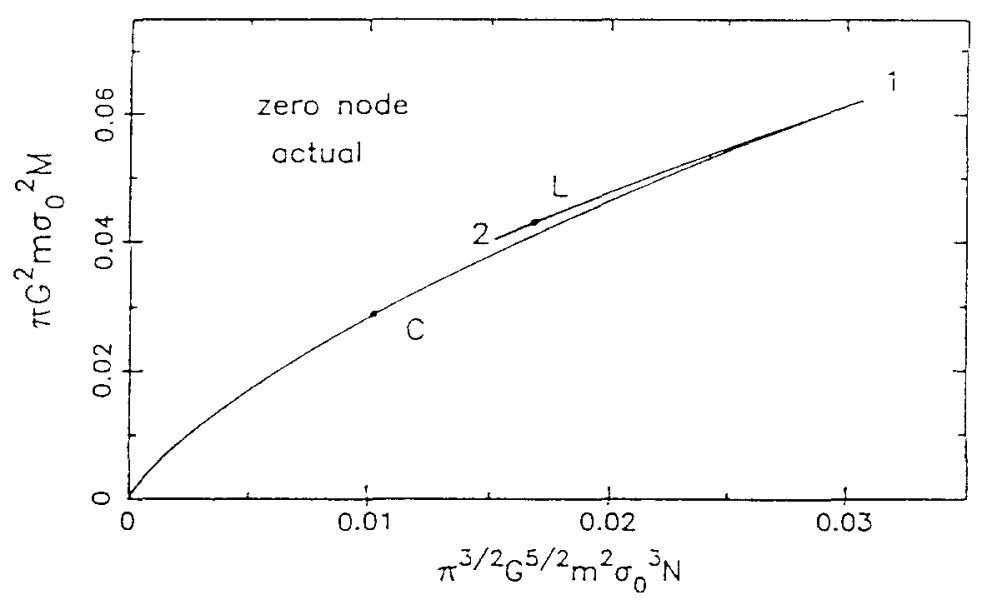

(b)

Fig. 22. Soliton star mass $M$ versus the particle number $N$ : a schematic drawing in (a) and the actual plot in (b).

type of nontopological soliton, the mini-boson star, which is the solution of $(6.57)-(6.60)$, regular everywhere and with

$$
\omega<m
$$

Therefore, at $\infty$ we have

$$
\sigma=\mathrm{O}\left(\exp \left[-\left(m^{2}-\omega^{2}\right)^{1 / 2} \rho\right]\right) \rightarrow 0
$$

Likewise, $U, V$ and $W$ all $\rightarrow 0$ exponentially at $x$. Thus, $u$ and $v$ approach the Schwarzschild solution also exponentially, as $\rho \rightarrow \infty$,

$$
\mathrm{e}^{u} \rightarrow(1-4 a / \rho)^{1 / 2}, \quad \mathrm{e}^{v} \rightarrow(1-4 a / \rho)^{-1 / 2}, \text { where } a=\frac{1}{2} G M
$$



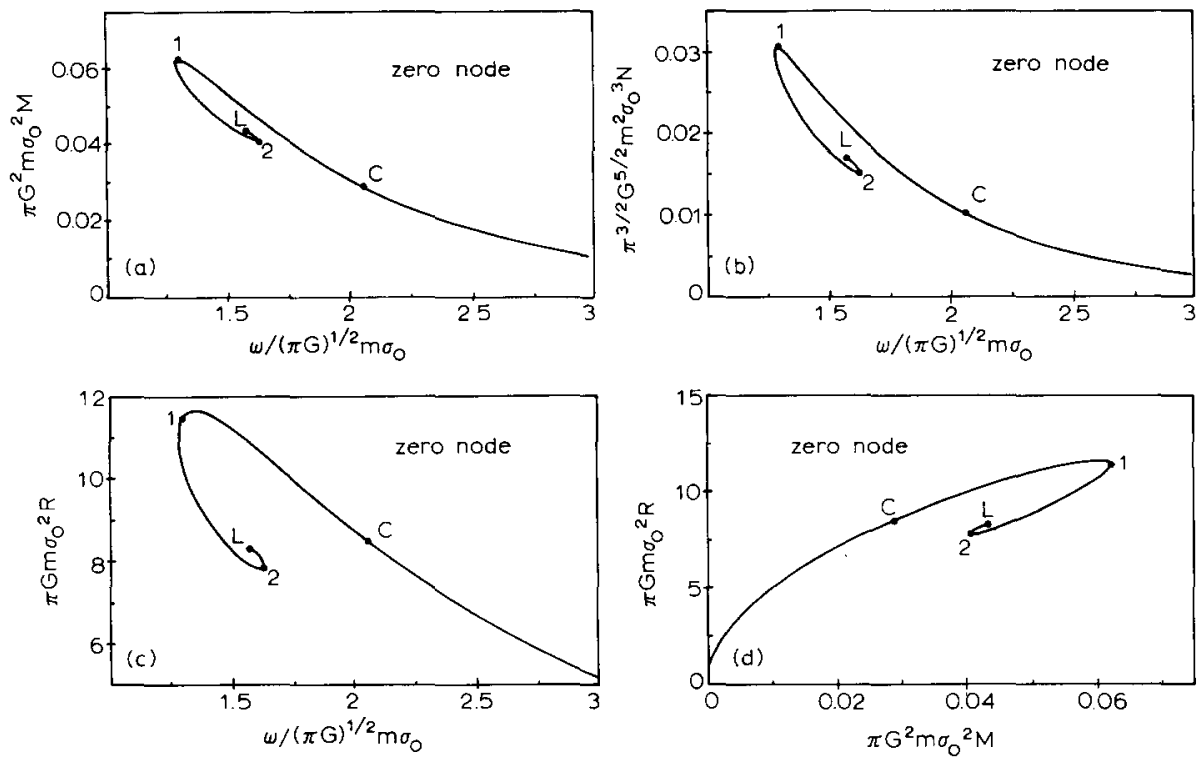

Fig. 23. (a) $M$ versus $\omega$, (b) $N$ versus $\omega$, (c) $R$ versus $\omega$, and (d) $R$ versus $M$.

When $\omega$ is only slightly less than $m$, such that

$$
\xi \equiv\left(1-\omega^{2} / m^{2}\right)^{1 / 2} \ll 1,
$$

the deviations of the metric $\mathrm{e}^{2 u}$ and $\mathrm{e}^{2 v}$ from 1 are also small, i.e.

$$
|u| \ll 1, \quad|v| \ll 1
$$
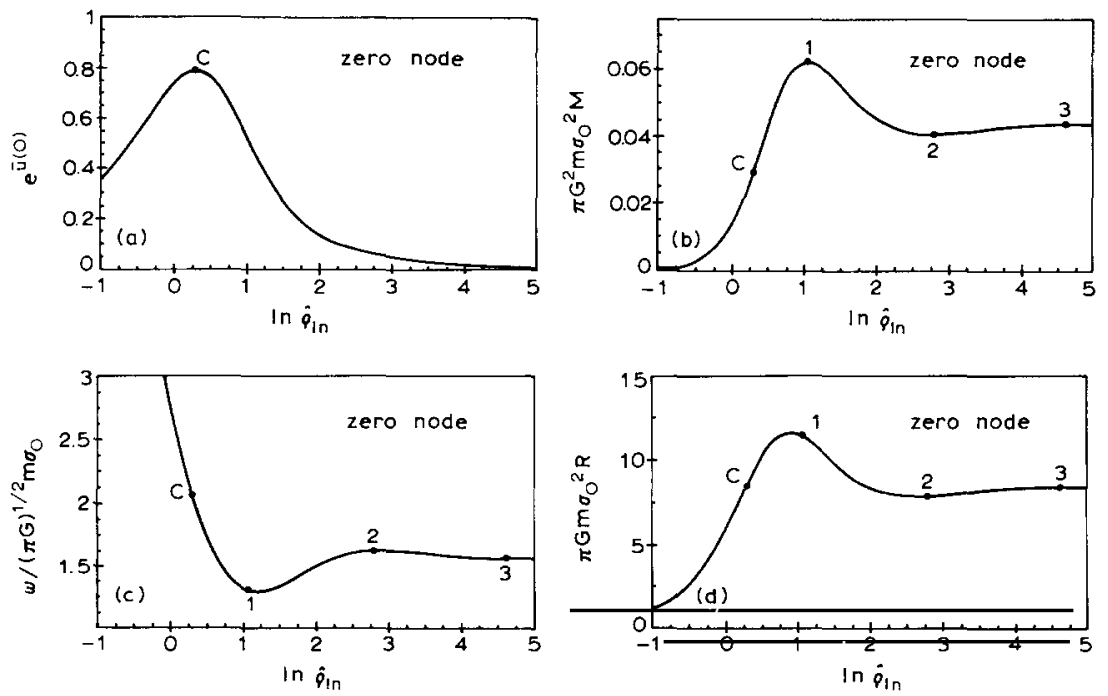

Fig. 24. (a) $\mathrm{e}^{\bar{u}(0)}$ versus $\hat{\rho}_{\text {in }}$, (b) $M$ versus $\hat{\rho}_{\text {in }}$, (c) $\omega$ versus $\hat{\rho}_{\text {in }}$, and (d) $R$ versus $\hat{\rho}_{\text {in }}$. 
This is the Newtonian limit of the mini-boson star. In this limit, we can neglect $u^{2}$ and $v^{2} ;(6.57)-(6.59)$ become

$$
\begin{aligned}
& 2 v+2 \rho \mathrm{d} v / \mathrm{d} \rho=8 \pi G \rho^{2}(W+V+U), \\
& -2 v+2 \rho \mathrm{d} u / \mathrm{d} \rho=8 \pi G \rho^{2}(W+V-U), \\
& \mathrm{d}^{2} u / \mathrm{d} \rho^{2}+\rho^{-1}(\mathrm{~d} u / \mathrm{d} \rho-\mathrm{d} v / \mathrm{d} \rho)=8 \pi G(W-V-U) .
\end{aligned}
$$

For $\omega<m, \sigma$ decreases with increasing $\rho$. Approximately, $\sigma$ is proportional to

$$
\exp \left[-\left(m^{2}-\omega^{2}\right)^{1 / 2} \rho\right]=\mathrm{e}^{-\xi m \rho}
$$

Thus, the radial size of the mini-star is $\sim(\xi m)^{-1}$ and a typical $\rho$ derivative $\mathrm{d} / \mathrm{d} \rho$ brings a factor $\xi m$, i.e.

$$
\mathrm{d} / \mathrm{d} \rho \sim \xi m
$$

and therefore we may estimate

$$
(m \sigma)^{-1} \mathrm{~d} \sigma / \mathrm{d} \rho=\mathrm{O}(\xi)
$$

For the same reason, $V$ is small compared to $U$ by a factor $\sim \xi^{2}$. Since $\omega$ is near $m, W$ differs from $U$ by a similar factor, i.e.

$$
V / U=\mathrm{O}\left(\xi^{2}\right), \quad(U-W) / U=\mathrm{O}\left(\xi^{2}\right)
$$

Multiplying $\mathrm{d} \sigma / \mathrm{d} \rho$ to the equation (6.60) we get, without approximation,

$$
(\mathrm{d} / \mathrm{d} \rho)(W+V-U)=-(4 / \rho) V-2(W+V) \mathrm{d} u / \mathrm{d} \rho
$$

Substituting (6.150)-(6.152) in the above equation, we see that the left-hand side is $\mathrm{O}\left(m \xi^{3} U\right)$ and the right-hand side is $\mathrm{O}(U m \xi u)$. Thus,

$$
u=\mathrm{O}\left(\xi^{2}\right)
$$

From (6.146), we estimate

$$
v=\mathrm{O}\left(G U / \xi^{2} m^{2}\right)
$$

Combining (6.154), (6.155) and (6.152), we find (6.148) to be consistent with

$$
G U / m^{2} \xi^{2}=\mathrm{O}\left(\xi^{2}\right)
$$

Therefore 


$$
G U / m^{2}=\mathrm{O}\left(\xi^{4}\right), \quad v=\mathrm{O}\left(\xi^{2}\right)
$$

Neglecting $\xi^{4}$, from the sum $(6.146)+(6.147)$, we have

$$
\mathrm{d}(v+u) / \mathrm{d} \rho=8 \pi G \rho W .
$$

Combining (6.154), (6.157) and (6.140) with (6.60), we derive, accurate up to $\mathrm{O}\left(\xi^{2} m^{2} \sigma\right)$,

$$
\mathrm{d}^{2} \sigma / \mathrm{d} \rho^{2}+(2 / \rho) \mathrm{d} \sigma / \mathrm{d} \rho=\left(m^{2}-\omega^{2} \mathrm{e}^{-2 u}\right) \sigma .
$$

Likewise, $\frac{1}{2}[(6.146)+(6.147)]+(6.148)$ becomes

$$
\mathrm{d}^{2} u / \mathrm{d} \rho^{2}+(2 / \rho) \mathrm{d} u / \mathrm{d} \rho=4 \pi G \omega^{2} \sigma^{2},
$$

which is accurate up to $\mathrm{O}\left(\xi^{4} m^{2}\right)$. Define

$$
\gamma \equiv 1-\left(\omega^{2} / m^{2}\right) \mathrm{e}^{-2 u}
$$

Equations (6.159) and (6.160) can be written as

$$
\nabla^{2} \sigma=\gamma m^{2} \sigma, \quad \nabla^{2} \gamma=8 \pi G m^{2} \sigma^{2}, \text { where } \nabla^{2}=\frac{\mathrm{d}^{2}}{\mathrm{~d} \rho^{2}}+\frac{2}{\rho} \frac{\mathrm{d}}{\mathrm{d} \rho}
$$

These equations can also be derived directly by using Newtonian gravity.

Introducing

$$
\gamma=\lambda^{2} \hat{\gamma}, \quad \rho=(m \lambda)^{-1} \hat{\rho}, \quad \sigma=(8 \pi G)^{-1 / 2} \lambda^{2} \hat{\sigma}, \quad \hat{\nabla}^{2} \equiv \frac{\mathrm{d}^{2}}{\mathrm{~d} \hat{\rho}^{2}}+\frac{2}{\hat{\rho}} \frac{\mathrm{d}}{\mathrm{d} \hat{\rho}},
$$

we convert (6.162) into a set of scale independent equations:

$$
\hat{\nabla}^{2} \hat{\sigma}=\hat{\gamma} \hat{\sigma}, \quad \hat{\nabla}^{2} \hat{\gamma}=\hat{\sigma}^{2}
$$

The solution of $\hat{\sigma}(\hat{\rho})$ can be characterized by its number of nodes $n$. The ground state corresponds to $n=0$. It is convenient to fix (for any $n$ )

$$
\hat{\sigma}=1 \quad \text { at } \quad \hat{\rho}=0
$$

These solutions are then universal functions. The special cases $n=0$ and 5 are given in fig. 25 as examples.

Take the ground state solution, $n=0$. From the numerical results, we find

$$
\hat{\gamma} \rightarrow \hat{\gamma}_{0}=-0.91858 \quad \text { as } \quad \hat{\rho} \rightarrow 0, \quad \hat{\gamma} \rightarrow \hat{\gamma}_{\infty}-\left(\hat{\gamma}_{1} / \hat{\rho}\right) \quad \text { as } \quad \hat{\rho} \rightarrow \infty,
$$

where $\hat{\gamma}_{\infty}=0.97896, \hat{\gamma}_{1}=3.46826$. 

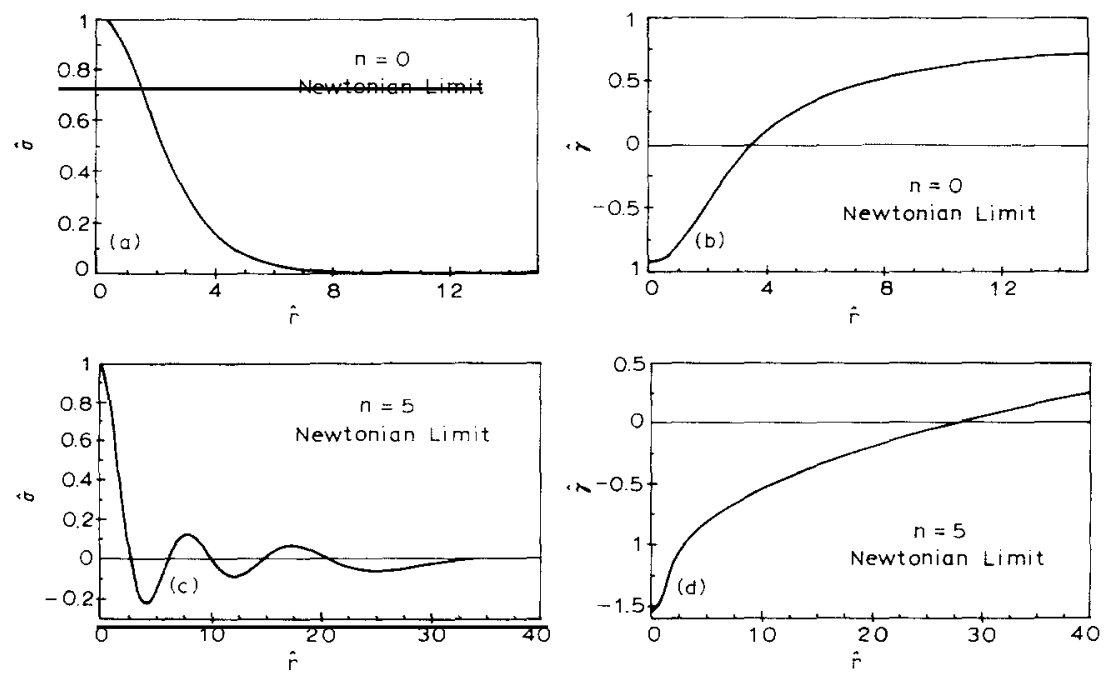

Fig. 25. Newtonian limit of the gravitational potential and the matter amplitude when the number of nodes $n=0$ and 5 .

The asymptotic behavior of the matter field $\hat{\sigma}$ is given by

$$
\hat{\sigma} \rightarrow \hat{\sigma}_{x} \exp \left(-\hat{\gamma}_{x}^{1 / 2} \hat{\rho}\right) \hat{\rho}^{\prime \prime} \text { as } \hat{\rho} \rightarrow x
$$

where

$$
\sigma_{x}=3.3943,2(\nu+1)=\hat{\gamma}_{1} / \hat{\gamma}_{x}
$$

Once $\hat{\gamma}$ and $\hat{\sigma}$ are known, $\gamma$ and $\sigma$ can be obtained from (6.163), in which $\lambda^{2}$ is related to $\omega / m$ by taking the $\rho=\infty$ limit of the first equation, $\gamma=\lambda^{2} \hat{\gamma}$. We have

$$
\xi^{2}=1-\omega^{2} / m^{2}=\lambda^{2} \hat{\gamma}_{x}
$$

The particle number is

$$
N=4 \pi \int_{0}^{\infty} \omega \sigma^{2} \mathrm{e}^{-u+v} \rho^{2} \mathrm{~d} \rho \simeq\left(2 G m^{2}\right)^{-1} \lambda \int \hat{\sigma}^{2} \hat{\rho}^{2} \mathrm{~d} \hat{\rho}
$$

where the integral is related to the asymptotic behavior (6.166) of $\hat{\gamma}$, through Gauss' theorem. We find

$$
N=\left(2 G m^{2}\right)^{-1} \hat{\gamma}_{\nsim}^{-1 / 2} \gamma_{1}\left(1-\omega^{2} / m\right)^{1 / 2} .
$$

Let

$$
\omega=m(1-\varepsilon)
$$


For small $\varepsilon,(6.170)$ becomes

$$
N=N_{0} \varepsilon^{1 / 2}, \text { where } N_{0}=\left(2 G m^{2}\right)^{-1}\left(2 / \hat{\gamma}_{\infty}\right)^{1 / 2} \hat{\gamma}_{1} .
$$

Because of $\mathrm{d} M / \mathrm{d} N=\omega$ and (6.172),

$$
\mathrm{d} M / \mathrm{d} N=m(1-\varepsilon)=m\left[1-\left(N / N_{0}\right)^{2}\right],
$$

which gives

$$
M=N m\left(1-\frac{1}{3} \varepsilon\right) \text {. }
$$

The values of $\hat{\gamma}_{0}, \hat{\gamma}_{\infty}$ and $\hat{\gamma}_{1}$ for $n=0,1,2,3,4,5$ are given in table 4 .

As $N$ increases, so does $\mathrm{e}^{-\bar{u}(0)}$, therefore the Newtonian limit is no longer a good approximation. On the $M$ versus $N$ curve, fig. 26 shows that for the exact solution there are many cusps, while the Newtonian limit (6.175) does not have any cusp. Figure 26 is obtained by integrating equations (6.57)-(6.60) numerically with $U$ given by (6.140). The numerical integrations are carried out in terms of dimensionless quantities

$$
\bar{\rho} \equiv m \rho, \quad \bar{\sigma} \equiv(16 \pi G)^{1 / 2} \sigma .
$$

In addition we define

$$
\bar{U} \equiv \frac{1}{2} \bar{\sigma}^{2}, \quad \bar{V} \equiv \frac{1}{2} \mathrm{e}^{-2 v}(\mathrm{~d} \bar{\sigma} / \mathrm{d} \bar{\rho})^{2}, \quad \bar{W} \equiv \frac{1}{2} \mathrm{e}^{-2 \bar{u}} \bar{\sigma}^{2},
$$

where

$$
\mathrm{e}^{-\bar{u}} \equiv(\omega / m) \mathrm{e}^{-u}
$$

Equations (6.57), (6.58) and (6.60) can be reduced to

$$
\begin{aligned}
& \mathrm{e}^{-2 v}-1-2 \bar{\rho} \mathrm{e}^{-2 v} \bar{\rho} \mathrm{d} v / \mathrm{d} \bar{\rho}=-\frac{1}{2}(\bar{W}+\bar{V}+\bar{U}) \bar{\rho}^{2} \\
& \mathrm{e}^{-2 v}-1+2 \bar{\rho} \mathrm{e}^{-2 v} \bar{\rho} \mathrm{d} \bar{u} / \mathrm{d} \bar{\rho}=\frac{1}{2}(\bar{W}+\bar{V}-\bar{U}) \bar{\rho}^{-2} \\
& \mathrm{e}^{-2 v}\left[\frac{\mathrm{d}^{2} \bar{\sigma}}{\mathrm{d} \bar{\rho}^{2}}+\left(\frac{2}{\bar{\rho}}+\frac{\mathrm{d} \bar{u}}{\mathrm{~d} \bar{\rho}}-\frac{\mathrm{d} \bar{\sigma}}{\mathrm{d} \bar{\rho}}\right) \frac{\mathrm{d} \bar{\sigma}}{\mathrm{d} \bar{\rho}}\right]=\left(1-\mathrm{e}^{-2 \bar{u}}\right) \bar{\sigma}
\end{aligned}
$$

Table 4

Values of $\hat{\gamma}_{0}, \hat{\gamma}_{x}$, and $\hat{\gamma}_{1}$ for the newtonian solutions when the number of nodes $n=0,1$,

\begin{tabular}{lllr}
\multicolumn{4}{c}{$2, \ldots, 5[25]$} \\
\hline$n$ & $\hat{\gamma}_{0}$ & $\hat{\gamma}_{x}$ & \multicolumn{1}{c}{$\hat{\gamma}_{1}$} \\
\hline 0 & -0.91858 & 0.97896 & 3.46826 \\
1 & -1.20996 & 0.91627 & 7.71395 \\
2 & -1.34370 & 0.89221 & 11.93547 \\
3 & -1.42828 & 0.87799 & 16.13218 \\
4 & -1.48943 & 0.86812 & 20.31019 \\
5 & -1.53701 & 0.86066 & 24.47366 \\
\hline
\end{tabular}




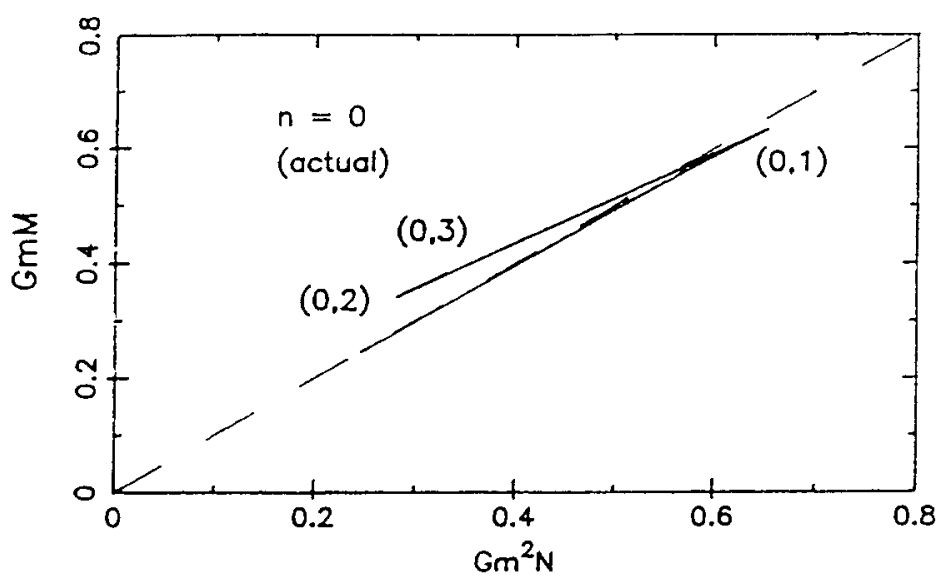

(a)

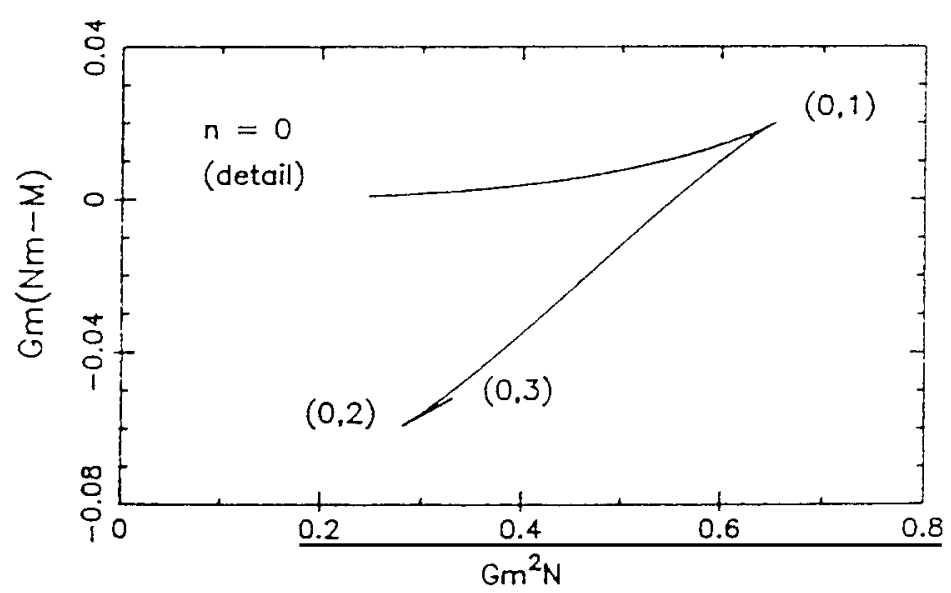

(b)

Fig. 26. Actual plot of (a) the mass $M$ versus the particle number $N$ and (b) the binding energy $N m-M$ versus $N$, for $n=0$.

The boundary conditions at the origin $\bar{\rho}=0$ are

$$
\bar{u}=\bar{u}(0), \quad v=0, \quad \bar{\sigma}=\bar{\sigma}(0) .
$$

With any given values of $\bar{u}(0)$ and $\bar{\sigma}(0)$, we can integrate $(6.179)-(6.181)$ from $\bar{\rho}=0$ outwards. The boundary conditions at $\bar{\rho}=\infty,(6.142)$ and (6.143), most likely will not be satisfied. By adjusting either $\bar{u}(0)$ or $\bar{\sigma}(0)$ until we satisfy the boundary condition as $\bar{\rho} \rightarrow \infty$, we obtain a mini-boson star solution.

For very large $\mathrm{e}^{-\bar{u}(0)}$, although the curves $M$ versus $N, N$ versus $\omega$ and $\omega$ versus $\mathrm{e}^{-\bar{u}}$ all keep on oscillating, the values of $M, N$ and $\omega$ hardly deviate at all from their asymptotic limits. These oscillations can be understood by considering the $(x, y)$ trajectories of the mini-boson stars. The boundary conditions $(6.182)$ give us 


$$
\mathrm{d} y /\left.\mathrm{d} x\right|_{\bar{\rho}=0}=[\bar{U}(0)+\bar{W}(0)] /[2 \bar{U}(0)-4 \bar{W}(0)] \leq-\frac{1}{4},
$$

where $\bar{U}(0)$ and $\bar{W}(0)$ are the values of $\bar{U}$ and $\bar{W}$ at $\bar{\rho}=0$.

As examples, we give the $(x, y)$ trajectories for $n=0$ and 1 in fig. $27, n=10$ in fig. $28 \mathrm{~d}$, all for the initial value

$$
\mathrm{e}^{-\bar{u}(0)}=2
$$

The Schwarzschild hyperbola is always represented by the dashed curves in these figures. Further illustrations of $\sigma, x$ and $y$ versus $\rho$ are given in figs. 28a-28c, for $n=10$. Detailed analysis of these solutions is given in ref. [25].

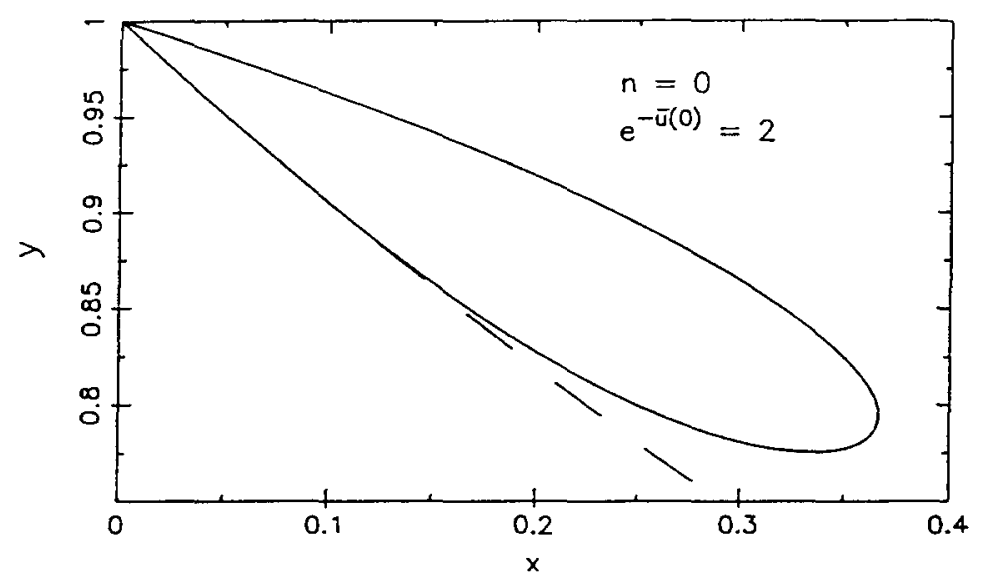

(o)

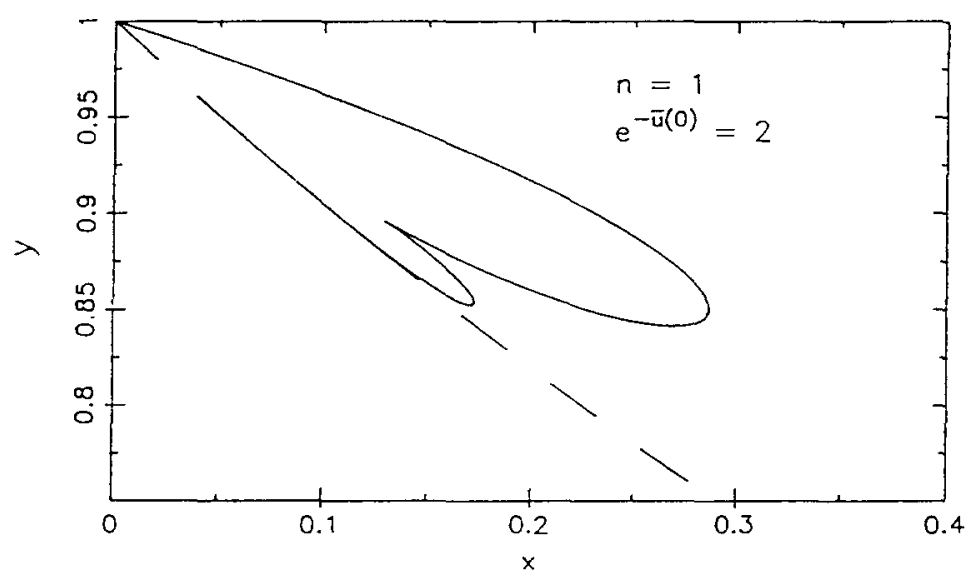

(b)

Fig. 27. The initial $(x, y)$ trajectory of the solution with an initial value $\mathrm{e}^{\bar{u}(0)}=2$, (a) for $n=0$ and (b) for $n=1$. The dashed curve is the Schwarzschild hyperbola. 

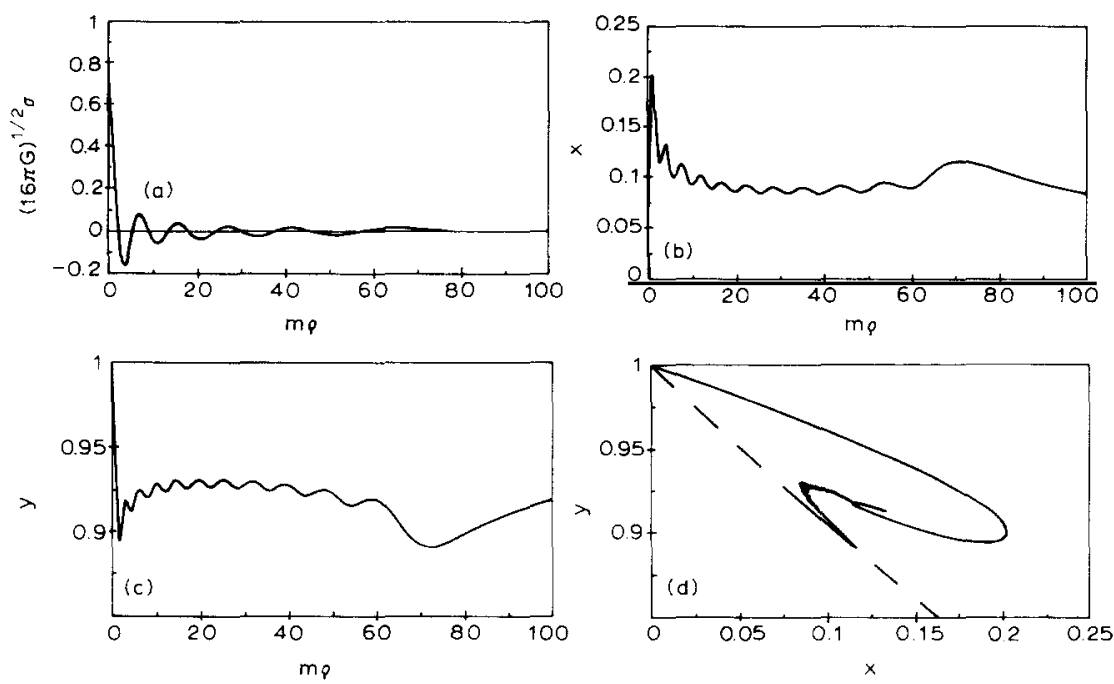

Fig. 28. Solution for a mini-soliton star solution with nodes $(n=10)$ and an initial value $\mathrm{e}^{(1+1)}=2$.

Table 5 gives the values of radius $R$, particle number $N$ and mass $M$ at the first cusp $(n, 1)$ when the number of nodes $n=0,1, \ldots, 10$, where the radius $R$ of a mini-star is defined by

$$
R \equiv \frac{4 \pi}{M} \int_{0}^{\infty}(U+V+W) \rho^{3} \mathrm{~d} \rho .
$$

This definition of $R$ makes use of the mass formula (6.82).

In this section, we examine only the minimal gravitational coupling between the scalar field and gravity. Of course soliton solutions can also exist with nonminimal gravitational couplings [124].

Gravity plays a pivotal role in the stability of a mini-boson star. Unlike boson stars, without gravity the mini-boson star does not exist. So we do not have a zero-gravity limit to help us. The stability of mini-boson stars under spherical perturbations was established for the lowest energy zero node

Table 5

Radius $R$, particle number $N$, and mass $M$ at the first cusp $(n, 1)$ when the number of nodes $n=0.1 \ldots 10[25 \mid$.

\begin{tabular}{rrrr}
\hline \multicolumn{1}{c}{$n$} & $m R$ & $G m^{2} N$ & $G m M$ \\
\hline 0 & 3.10978 & 0.653003 & 0.633001 \\
1 & 7.89162 & 1.3921 .34 & 1.356265 \\
2 & 12.83169 & 2.137840 & 2.085372 \\
3 & 17.84001 & 2.883631 & 2.814529 \\
4 & 22.89276 & 3.628891 & 3.543186 \\
5 & 27.97750 & 4.373581 & 4.271317 \\
6 & 33.08911 & 5.117754 & 4.998970 \\
7 & 38.22251 & 5.861469 & 5.726202 \\
8 & 43.37470 & 6.604782 & 6.453063 \\
9 & 48.54421 & 7.347736 & 7.179595 \\
10 & 53.72830 & 8.090369 & 7.905833 \\
\hline
\end{tabular}


solutions [56]. All other solutions are not stable under these perturbations [56, 125, 126]. The proof is nontrivial. Besides the stability against fission into several smaller mini-boson stars and the decay into plane wave solutions, one must consider the complications due to black holes. For details, see ref. [56].

\subsection{Fermion soliton stars [27]}

The necessary and sufficient conditions for fermion soliton stars are (i) the conservation of the fermion number $N$ and (ii) the existence of nontopological soliton solutions, in the absence of the gravitational field. It is the latter condition that distinguishes a soliton star from a neutron star, or a white dwarf.

In order to satisfy (ii), we assume the existence of a real (hermitian) scalar field $\sigma$, in addition to the fermion field $\psi$ and the gravitational field. The simplest example is the Friedberg-Lee model of section 5 without the gauge field. We choose the self interaction of $\sigma$ to have the degenerate vacuum form

$$
U(\sigma)=\frac{1}{2} \mu^{2} \sigma^{2}\left(1-\sigma / \sigma_{0}\right)^{2}
$$

with $\mu=\sigma$-mass. We may assign $\sigma=0$ to be the normal vacuum state, and $\sigma=\sigma_{0}$ the false (or degenerate) vacuum state. The interaction between $\sigma$ and $\phi$ is

$$
-f \bar{\psi} \psi \sigma
$$

where $f$ is the coupling and $\bar{\psi}$ is the adjoint of $\psi$, making $\bar{\psi} \psi$ a Lorentz scalar. Let the fermion mass (in the normal vacuum) be $m$. For simplicity, we further assume

$$
m-f \sigma_{0}=0
$$

so that the fermion has zero effective mass in the false vacuum.

For the fermion field $\psi$, we shall adopt the Thomas-Fermi approximation. At each point in space there is a Fermi momentum $k_{\mathrm{F}}$ (observed in the appropriate local frame) which, for the spherically symmetric solution, depends only on $\rho$, or equivalently only on $r$. The fermion energy density is given by the familiar expression

$$
W=\frac{2}{8 \pi^{3}} \int \mathrm{d}^{3} k n_{k} \varepsilon_{k},
$$

where the factor of 2 is due to the spin degeneracy $\int \mathrm{d}^{3} k=4 \pi \int k^{2} \mathrm{~d} k, n_{k}$ is the Fermi distribution

$$
n_{k}=\theta\left(k-k_{\mathrm{F}}\right)= \begin{cases}1 & \text { if } k<k_{\mathrm{F}}, \\ 0 & \text { if } k>k_{\mathrm{F}}\end{cases}
$$

and the energy

$$
\varepsilon_{k}=\left[k^{2}+(m-f \sigma)^{2}\right]^{1 / 2}
$$

The corresponding fermion number density $\nu$ and the nonzero components of the stress tensor $T_{\nu}^{\mu}$ of the 
fermion field are

$$
\begin{aligned}
& \nu=\frac{2}{8 \pi^{3}} \int \mathrm{d}^{3} k n_{k}, \\
& T_{t}^{i}=W, \quad T_{r}^{r}=T^{\alpha}=T_{\beta}^{\beta}=T_{p}^{\rho}=-T, \text { where } T=\frac{2}{8 \pi^{3}} \int \mathrm{d}^{3} k n_{k} \frac{k^{2}}{3 \varepsilon_{k}} .
\end{aligned}
$$

Consequently, they satisfy the identities

$$
T_{\mu}^{\mu}=W-3 T=(m-f \sigma) S, \quad W-T=\varepsilon_{\mathrm{f}} \nu,
$$

where $S$ is the scalar density $\bar{\psi} \psi$ in the Thomas-Fermi approximation,

$$
S=\frac{2}{8 \pi^{3}} \int \mathrm{d}^{3} k n_{k} \varepsilon_{k}^{-1}(m-f \sigma) \text {. }
$$

The total fermion number $N$ and the total fermion energy $U(f)$ are given by

$$
N=4 \pi \int \mathrm{e}^{v} \rho^{2} \mathrm{~d} \rho \nu, \quad E(f)=4 \pi \int \mathrm{e}^{u+v} \rho^{2} \mathrm{~d} \rho W
$$

The total energy of the system consists of, besides $E(f)$, also the gravitational energy $E(g)$ and the $\sigma$ field energy $E(\sigma)$,

$$
E=E(f)+E(g)+E(\sigma) .
$$

The total energy density is

$$
T_{t}^{t}=W+V+U
$$

Other nonvanishing components of the total stress tensor are

$$
T_{\rho}^{\rho}=-(T+V-U), \quad T_{\alpha}^{\alpha}=T_{\beta}^{\beta}=-(T-V-U),
$$

where $V$ is defined, as in previous sections, to be

$$
V=\frac{1}{2} \mathrm{e}^{-2 v}(\mathrm{~d} \sigma / \mathrm{d} \rho)^{2}
$$

Regard the total energy $E$ as a function of $k_{\mathrm{F}}, \sigma, u$, and $v$. The basic equations can be obtained by taking the extreme of $E$ at a fixed fermion number $N$, i.e.

$$
\delta E-\omega_{\mathrm{F}} \delta N=0,
$$

where $\omega_{\mathrm{F}}$ is the Lagrange multiplier. This leads to the Einstein equations

$$
G_{,}^{\mu}=-8 \pi G T^{\mu},
$$


the equation for $k_{\mathrm{F}}$,

$$
\left[k_{\mathrm{F}}^{2}+(m-f \sigma)^{2}\right]^{1 / 2} \mathrm{e}^{u}=\varepsilon_{\mathrm{F}} \mathrm{e}^{u}=\omega_{F}=\text { constant },
$$

and the $\sigma$ field equation

$$
\mathrm{e}^{-2 v}\left[\frac{\mathrm{d}^{2} \sigma}{\mathrm{d} \rho^{2}}+\left(\frac{2}{\rho}+\frac{\mathrm{d} u}{\mathrm{~d} \rho}-\frac{\mathrm{d} v}{\mathrm{~d} \rho}\right) \frac{\mathrm{d} \sigma}{\mathrm{d} \rho}\right]+f S-\frac{\mathrm{d} U}{\mathrm{~d} \sigma}=0
$$

As in the case of scalar soliton stars, for large fermion number $N$, the fermion soliton star solution can also be divided into three regions: the interior $(\rho<R)$, the surface $\left(R-\mathrm{O}\left(\mu^{-1}\right)<\rho<R+\right.$ $\left.\mathrm{O}\left(\mu^{-1}\right)\right)$, and the exterior $(\rho>R)$. In the interior, we may approximate

$$
\sigma=\sigma_{0}
$$

and consequently

$$
\mathrm{d} U / \mathrm{d} \sigma=(m-f \sigma)=S=0 .
$$

Similarly,

$$
U=V=0, \quad 3 T=W=k_{\mathrm{F}}^{4} / 4 \pi^{2},
$$

where $k_{\mathrm{F}}$ is the Fermi momentum.

Let us define a small parameter

$$
\lambda \equiv 4 \sigma_{0}(\pi G / 3)^{1 / 3}
$$

which for $\sigma_{0} \sim 30 \mathrm{GeV}$ is about $10^{-17}$. Introducing dimensionless quantities

$$
\bar{\rho}=\lambda^{2} \mu \rho, \quad \mathrm{e}^{\bar{u}} \equiv\left(3 / 8 \pi^{2}\right)^{1 / 4}\left[\omega_{\mathrm{F}} /\left(\lambda \mu \sigma_{0}\right)^{1 / 2}\right] \mathrm{e}^{-u}, \quad \bar{v}=v,
$$

we can write the equations for the interior as

$$
2 \bar{\rho} \frac{\mathrm{d} \bar{v}}{\mathrm{~d} \bar{\rho}}=\left(\mathrm{e}^{-4 \bar{u}} \bar{\rho}^{2}-1\right) \mathrm{e}^{2 \bar{v}}+1, \quad 2 \bar{\rho} \frac{\mathrm{d} \bar{u}}{\mathrm{~d} \bar{\rho}}=\left(\frac{1}{3} \mathrm{e}^{-4 \bar{u}} \bar{\rho}^{2}+1\right) \mathrm{e}^{2 \bar{v}}-1
$$

As before, it is convenient to express the solution in terms of the variables $x$ and $y$. The $W$ dominance in the interior enables us to eliminate $W$ in (6.201); the result is

$$
\frac{\mathrm{d} y}{\mathrm{~d} x}=\frac{-3 x y-2 y^{2}+2}{5 x y+3 y^{2}-x^{2}-3} .
$$

The initial slope, at $\rho=0$, is

$$
(\mathrm{d} y / \mathrm{d} x)_{0}=-\frac{1}{2}
$$




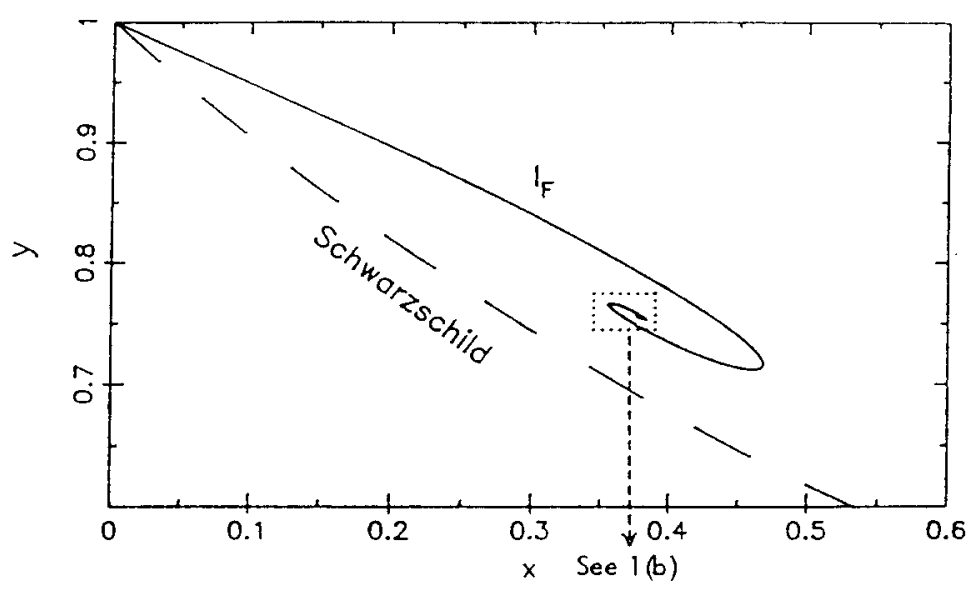

(a)

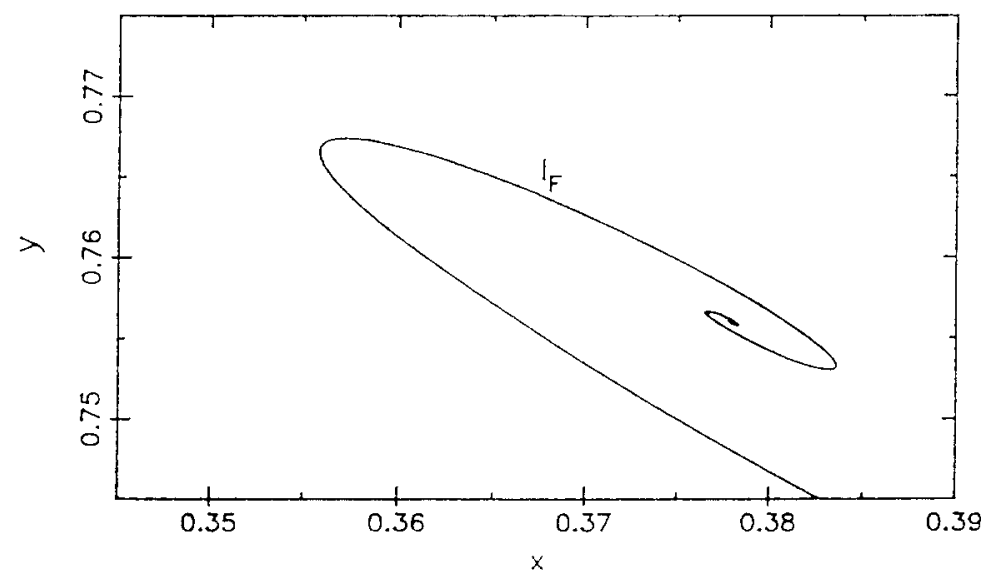

(b)

Fig. 29. Universal trajectory $I_{p}$. The dashed curve is the Schwarzschild hyperbola $2 x y+y^{2}-1=0$.

In fig. 29, the dashed curve is the Schwarzschild hyperbola, and the solid curve $\mathrm{I}_{\mathrm{F}}$ is the solution of (6.210) with the initial slope (6.211).

In the exterior region

$$
U=V=W=0
$$

the Schwarzschild solution takes over. In the surface region, it is more convenient to work in the isotropic coordinates. When $\rho=R$, denote $r=r_{\mathrm{s}}=R \mathrm{e}^{-\tilde{v}_{\mathrm{s}}}$. Within the surface, $x=r \mathrm{~d} u / \mathrm{d} r$ and $y=1+r \mathrm{~d} v / \mathrm{d} r$ are $\sim 1, \mathrm{~d} u / \mathrm{d} r$ and $\mathrm{d} v / \mathrm{d} r$ are both $\mathrm{O}\left(r_{\mathrm{s}}^{-1}\right)$. Hence, neglecting $\mathrm{O}\left(\lambda^{2}\right)$, we can regard $u=u_{\mathrm{s}}$ and $\tilde{v}=\tilde{v}_{\mathrm{s}}$ as constants across the surface; in addition, since $S \sim m k_{\mathrm{F}}^{2}=\lambda \mathrm{O}\left(m \mu^{2}\right)$, to the lowest 
order in $\lambda,(6.203)$ becomes

$$
\mathrm{e}^{-2 \tilde{v}} \mathrm{~d}^{2} \sigma / \mathrm{d} r^{2}-\mathrm{d} U / \mathrm{d} \sigma=0
$$

This gives the solution, valid for $r=r_{\mathrm{s}}+\mathrm{O}\left(\mu^{-1}\right)$,

$$
\sigma=\sigma_{0} /\left\{1+\exp \left[\mu \mathrm{e}^{\tilde{v}_{\mathrm{s}}}\left(r-r_{\mathrm{s}}\right)\right]\right\} .
$$

To the same accuracy, we have within the surface

$$
U=V=\mathrm{O}\left(\mu^{2} \sigma_{0}^{2}\right)
$$

but

$$
W=\mathrm{O}\left(\omega_{\mathrm{F}}^{2} m\right)=\lambda^{3 / 2} \mathrm{O}(U)
$$

By using (6.214), we find the integrals of $U$ and $V$ across the surface to be

$$
\int_{\text {surface }} U \mathrm{~d} r=\int_{\text {surface }} V \mathrm{~d} r=\frac{1}{12} \mu \sigma_{0}^{2} \mathrm{e}^{-\tilde{v}_{\mathrm{s}}} .
$$

Hence, we may approximate, in the surface region,

$$
U=V=\frac{1}{12} \mu \sigma_{0}^{2} \mathrm{e}^{-\tilde{v}_{\mathrm{s}}} \delta\left(r-r_{\mathrm{s}}\right), \quad W=0 .
$$

These lead to

$$
\mathrm{d} x / \mathrm{d} r=\mathrm{d} y / \mathrm{d} r=-\frac{2}{3} \pi G r_{\mathrm{s}} \mathrm{e}^{\tilde{\nu}_{\mathrm{s}}} \mu \sigma_{0}^{2} \delta\left(r-r_{\mathrm{s}}\right)
$$

Integrating (6.219) across the surface, we see that the discontinuities in $x$ and $y$ from the interior solution "in" to the Schwarzschild (exterior) solution "A" are

$$
\begin{aligned}
& \Delta x=x_{\text {in }}-x_{\mathrm{A}}=\frac{1}{8} \bar{\rho}_{\mathrm{in}}=\frac{2}{3} \pi G r_{\mathrm{s}} \mathrm{e}^{\tilde{v}_{\mathrm{s}}} \mu \sigma_{0}^{2}, \\
& \Delta y=y_{\text {in }}-y_{\mathrm{A}}=\frac{1}{8} \bar{\rho}_{\text {in }}=\frac{2}{3} \pi G r_{\mathrm{s}} \mathrm{e}^{\tilde{v}_{\mathrm{s}}} \mu \sigma_{0}^{2},
\end{aligned}
$$

where $\bar{\rho}_{\text {in }}=\lambda^{2} \mu R$. Since $x_{\mathrm{A}}$ and $y_{\mathrm{A}}$ are on the Schwarzschild hyperbola, we have

$$
2 x_{\mathrm{A}} y_{\mathrm{A}}+y_{\mathrm{A}}^{2}-1=0 \text {. }
$$

As for boson stars, because $\Delta x=\Delta y$, we have

$$
\frac{1}{8} \bar{\rho}_{\text {in }}=\frac{1}{3}\left\{x_{\text {in }}+2 y_{\text {in }}-\left[\left(x_{\text {in }}-y_{\text {in }}\right)^{2}+3\right]^{1 / 2}\right\} \equiv \Delta\left(x_{\text {in }}, y_{\text {in }}\right) \text {. }
$$

Figure 30 shows four examples of the $(x, y)$ trajectory for fermion soliton star solutions. 

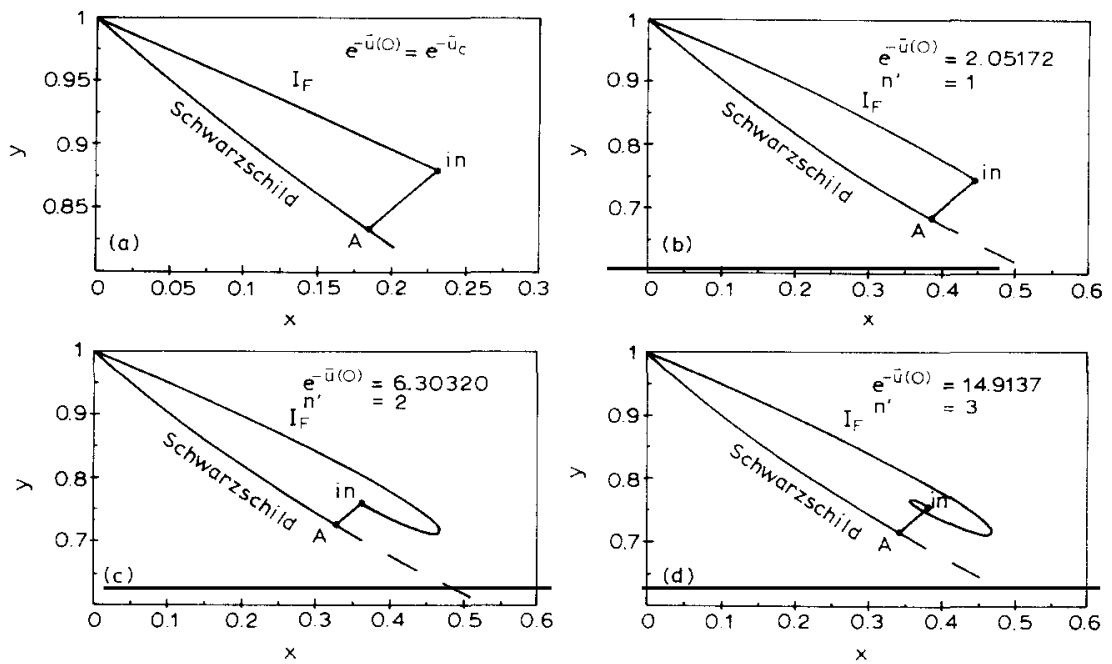

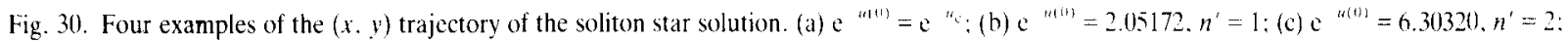
(d) $c^{2 " 0 t}=14.9137, n^{\prime}=3$.

Define $\hat{u}(\hat{\rho})$ and $\hat{v}(\hat{\rho})$ to be the solutions of

$$
2 \hat{\rho} \frac{\mathrm{d} \hat{v}}{\mathrm{~d} \hat{\rho}}=\left(\frac{1}{2} \mathrm{e}^{-4 \hat{\mu}} \hat{\rho}^{2}-1\right) \mathrm{e}^{2 \dot{v}}+1, \quad 2 \hat{\rho} \frac{\mathrm{d} \hat{u}}{\mathrm{~d} \hat{\rho}}=\left(\frac{1}{2} \mathrm{e}^{-4 \dot{\hat{\theta}}} \hat{\rho}^{2}+1\right) \mathrm{e}^{2 \hat{v}}-1 .
$$

with the boundary conditions

$$
\hat{u}(0)=\hat{v}(0)=0 \quad \text { at } \quad \hat{\rho}=0
$$

Any solution of (6.224) with boundary conditions $\bar{u}(0) \neq 0$ and $\bar{v}(0) \neq 0$ can then be derived from $\hat{u}(\hat{\rho})$ and $\hat{v}(\hat{\rho})$ through

$$
\mathrm{e}^{-\ddot{u}(\bar{\rho})}=\mathrm{e}^{-\dot{u}(\hat{\rho})-\bar{u}(1)}, \quad \mathrm{e}^{-\ddot{v}(\rho)}=\mathrm{e}^{-\dot{v}(\hat{\rho})}, \text { where } \bar{\rho}=\hat{\rho} \mathrm{e}^{2 \ddot{u}(0)} .
$$

The functions $\hat{u}(\hat{\rho})$ and $\hat{v}(\hat{\rho})$ are plotted in fig. 31 .

In fig. 32 the solid curve is $\Delta(\hat{\rho})$ which is independent of $\mathrm{e}^{-\bar{u}(0)}$, and the dashed straight line is

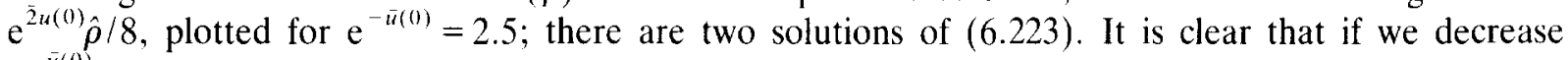
$\mathrm{e}^{-\bar{u}(0)}$, the dashed line will swing counterclockwise, until it reaches a critical point, called $c$, when

$$
\mathrm{e}^{-\bar{u}(0)}=\mathrm{e}^{-\bar{u}_{\mathrm{c}}}=1.620
$$

At c, eq. (6.223) has only one unique solution. The corresponding trajectory is given in fig. 30a.

For $\mathrm{e}^{-\bar{u}(0)}<\mathrm{e}^{-\bar{u}_{\mathrm{c}}}$, there is no solution; for $\mathrm{e}^{-\bar{u}(0)}>\mathrm{e}^{-\bar{u}_{\mathrm{c}}}$ there are two solutions. By systematically changing $\mathrm{e}^{-\bar{u}(0)}$, we can survey all the zero-node solutions $(n=0)$. In figs. 33a and $33 \mathrm{~b}, M$ is plotted versus $N$, schematically in 33a and precisely in 33b. In fig. 34, we show the curves $M, N$ and $R$ versus $\omega$ and $R$ versus $M$, and in fig. 35, the dependence of $\mathrm{e}^{\bar{u}(0)}, M, \omega$, and $R$ on $\hat{\rho}_{\text {in }}$. 

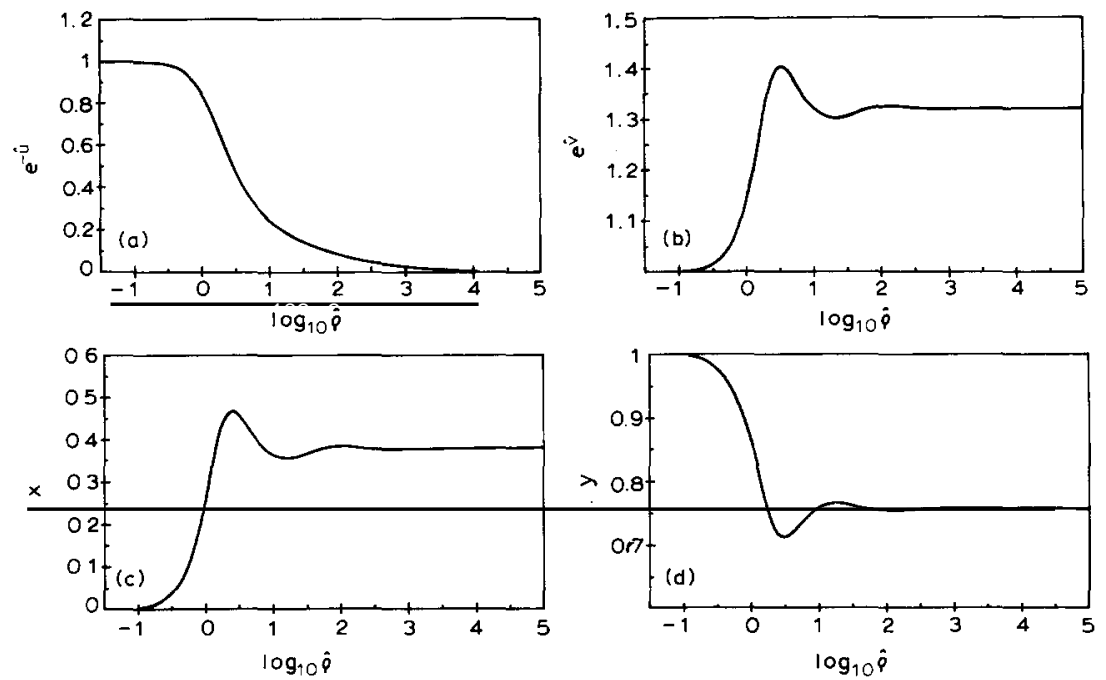

Fig. 31. From solutions of $\hat{u}(\hat{\rho}), \hat{v}(\hat{\rho}), x(\hat{\rho})$, and $y(\hat{\rho})$, one can derive $\bar{u}(\bar{\rho}), \bar{v}(\bar{\rho}), x(\bar{\rho})$ and $y(\bar{\rho})$ for any initial value $\bar{u}(0)$.

When $N$ is small and the effect of gravity is negligible, the stability condition has been discussed in section 3 for boson solitons and in section 5 for fermion solitons. The effect of gravity on stability has been examined in section 6.6 for the boson star. That result can be readily generalized to the fermion soliton star. Thus all fermion soliton star solutions on the lowest branch are stable under small perturbations.

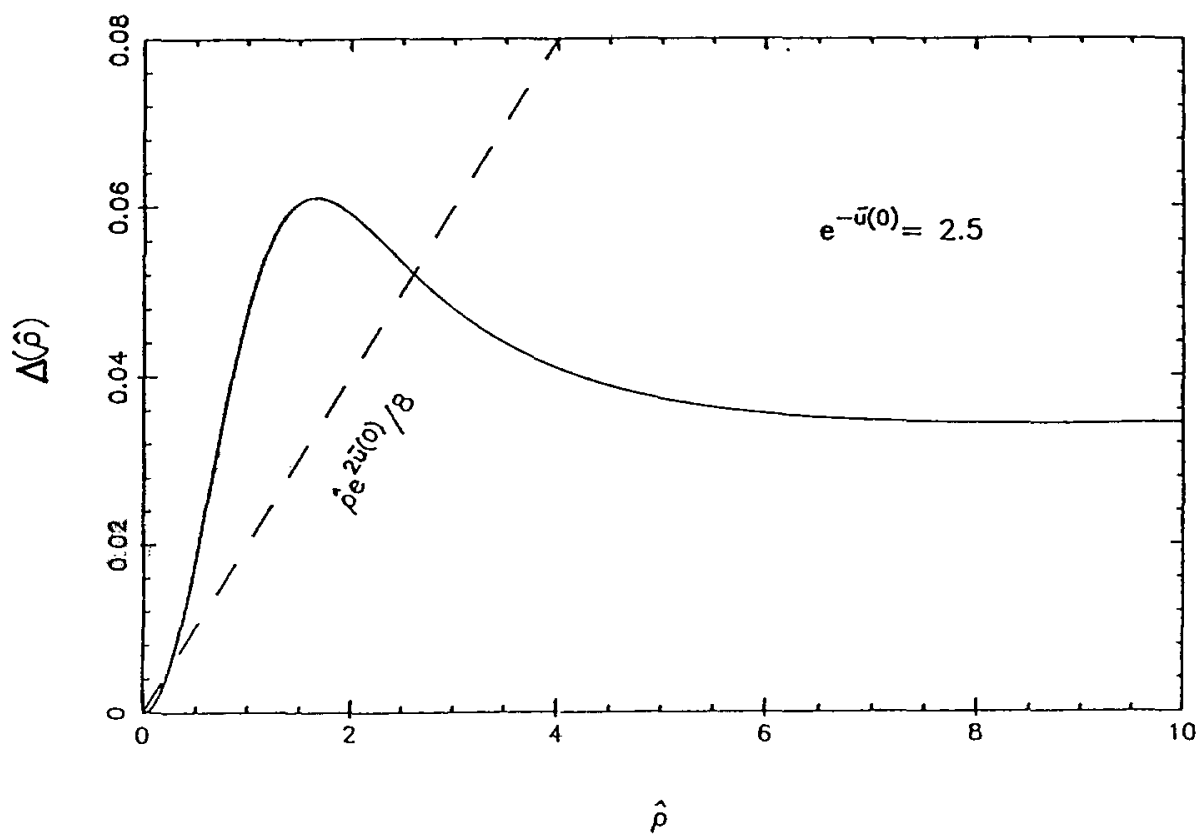

Fig. 32. The initial value $\mathrm{e}^{-\bar{u}(0)}$ determines the slope of the dashed line,$\frac{1}{8} \mathrm{e}^{\bar{u}(0)} \hat{\rho}$, whose intersection with $\Delta(\hat{\rho})$ gives a solution of the fermion soliton star. 


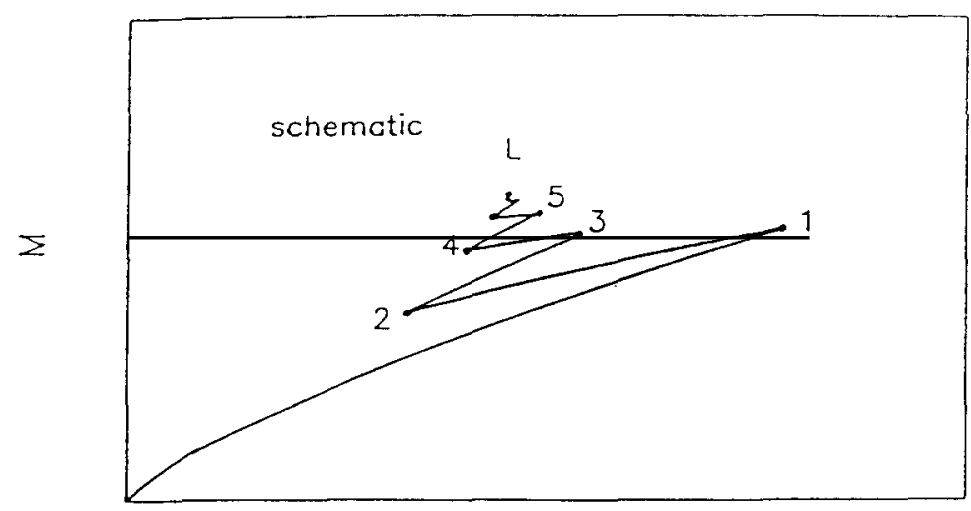

N

(a)

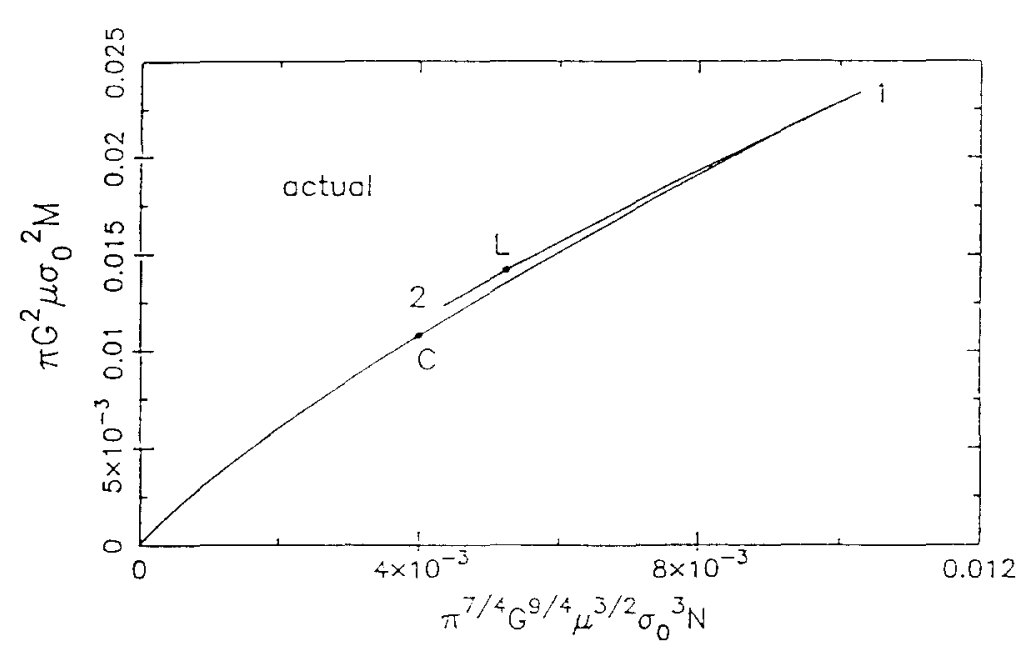

(b)

Fig. 33. Fermion soliton star mass $M$ versus the particle number $N$. (a) Schematic drawing. (b) actual plot.

Like the boson star, the maximum fermion star mass depends very sensitively on the form of $U$ [24]. With an appropriate choice of parameters, the fermion soliton star can have the size and mass of a neutron star [127-130], or it can be of the size and mass of a "quasar". If soliton stars exist, they may have been created in the early universe [131-134].

\subsection{Remark}

Recent progress in particle physics points out the importance of nonlinearity and coherence in the realm of $10^{-13}-10^{-17} \mathrm{~cm}$, as exemplified by the QCD vacuum in connection with quark confinement and the role of Higgs fields in electroweak symmetry breaking. Assuming that these overall phenomena may be effectively represented by scalar fields, it seems reasonable that such coherence can be 

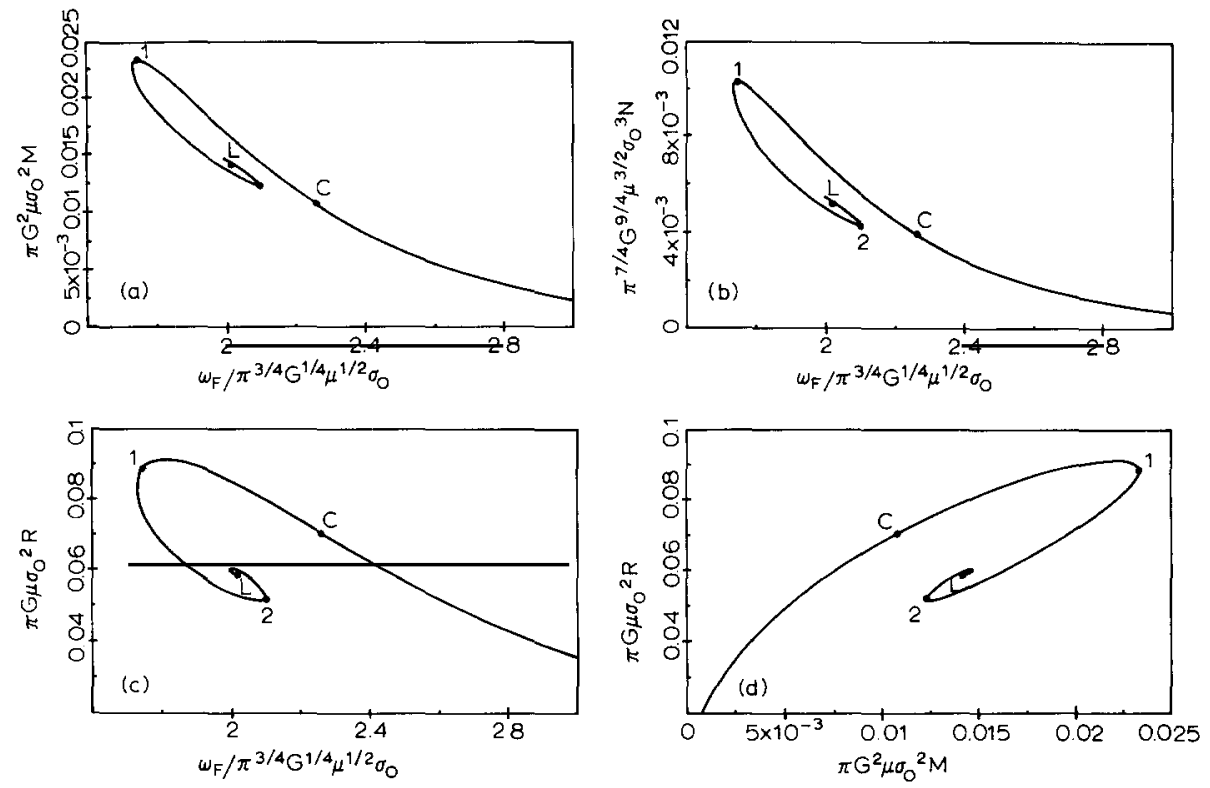

Fig. 34. (a) $M$ versus $\omega_{\mathrm{F}}$, (b) $N$ versus $\omega_{\mathrm{F}}$, (c) $R$ versus $\omega_{\mathrm{F}}$, and (d) $R$ versus $M$.

accumulated and extended to macroscopic and even to astronomical distances. The explicit model solutions derived here are meant to demonstrate the feasibility, at least in principle.

At present, there is no experimental evidence that soliton stars exist. Nevertheless, it seems reasonable that solutions of well-tested theories, such as Einstein's general relativity, the Dirac equation, the Klein-Gordon equation, etc. should find their proper place in nature.
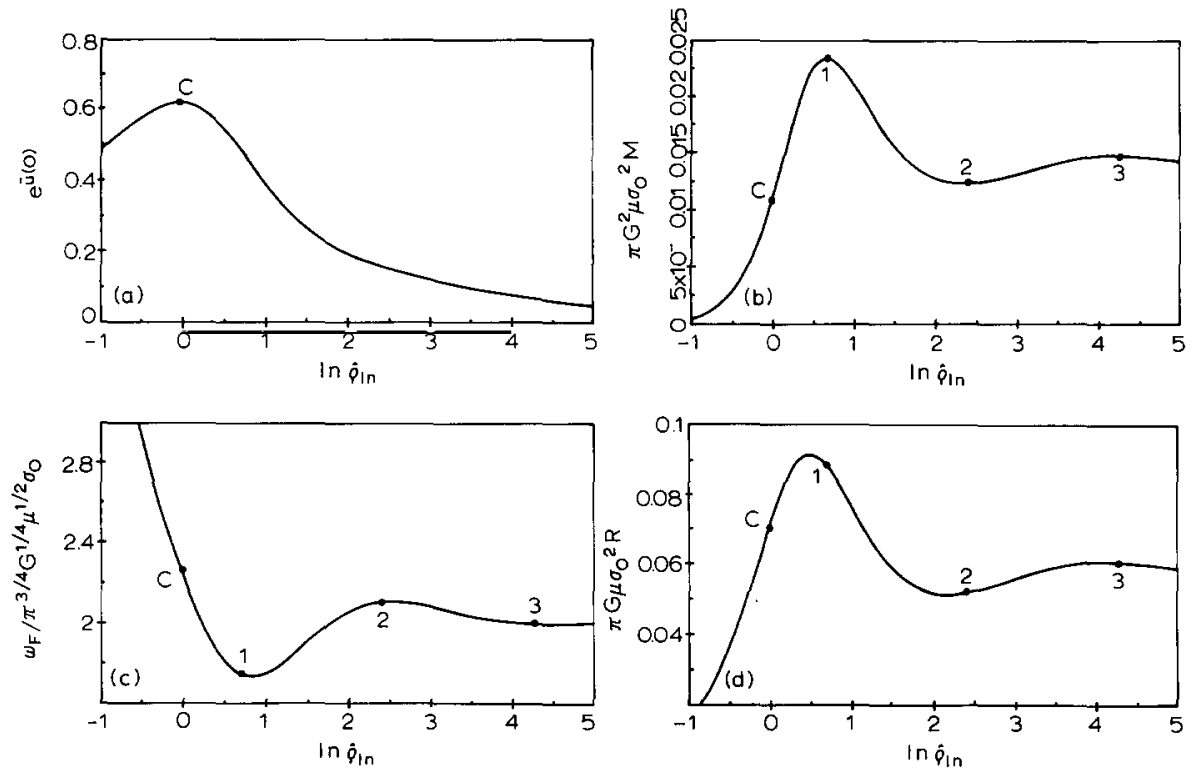

Fig. 35. (a) $\mathrm{e}^{\bar{u}(0)}$ versus $\hat{\rho}_{\text {in }}$, (b) $M$ versus $\hat{\rho}_{\text {in }}$, (c) $\omega$ versus $\hat{\rho}_{\text {in }}$, and (d) $R$ versus $\hat{\rho}_{\text {in }}$. 


\section{References}

[1] J.S. Russell, in: Report of the British Association for the Advancement of Science (1845).

[2] D.J. Korteweg and G. de Vries, Phil. Mag. 39 (1895) 422.

[3] G.H. Derrick, J. Math. Phys. 5 (1964) 1252.

[4] G. 't Hooft, Nucl. Phys. B 79 (1974) 276.

[5] A.M. Polyakov, JETP Lett. 20 (1974) 194.

[6] G. Rosen. J. Math. Phys. 9 (1968) 996, 999

[7] D.J. Kaup, Phys. Rev. 172 (1968) 1331.

[8] R. Ruffini and S. Bonazzola, Phys. Rev. 187 (1969) 1767.

[9] P. Vinciarelli, Nuovo Cimento Lett. 4 (1972) 905.

[10] T.D. Lee and G.C. Wick, Phys. Rev. D 9 (1974) 2291.

[11] A. Chodos, R.J. Jaffe, K. Johnson, C.B. Thorn and V.F. Weisskopf. Physa Rev. D 9 (1974) 3471.

[12] W.A. Bardeen, M.S. Chanowitz. S.D. Drell, M. Weinstein and T.M. Yan. Phys. Rev. D 11 (1975) 1094.

[13] R. Friedberg, T.D. Lee and A. Sirlin, Phys. Rev. D 13 (1976) 2739.

[14] R. Friedberg, T.D. Lee and A. Sirlin, Nucl. Phys. B 115 (1976) 1.

[15] R. Friedberg, T.D. Lee and A. Sirlin, Nucl. Phys. B 115 (1976) 32.

[16] S. Coleman, Nucl. Phys. B 262 (1985) 263.

[17] R. Friedberg and T.D. Lee, Phys. Rev. D 15 (1977) 1694.

[18] R. Friedberg and T.D. Lee. Phys. Rev. D 16 (1977) 1096.

[19] R. Friedberg and T.D. Lee, Phys. Rev. D 18 (1978) 2623.

[20] L. Wilets, Nontopological Solitons, Lecture Notes in Physics, Vol. 24 (World Scientific. Singapore. 1989).

[21] M. Birse, Soliton Models for Nuclear Physics, Manchester Univ. Preprint (1990).

[22] N.H. Christ and T.D. Lee, Phys. Rev. D 12 (1975) 1606.

[23] J. Goldstone and R. Jackiw, Phys. Rev. D 11 (1975) 1486.

[24] T.D. Lee, Phys. Rev. D 35 (1987) 3637; Comments Nucl. Particle Phys. XVII (1987) 225.

[25] R. Friedberg, T.D. Lee and Y. Pang, Phys. Rev. D 35 (1987) 3640.

[26] R. Friedberg. T.D. Lee and Y. Pang, Phys. Rev. D 35 (1987) 3658.

[27] T.D. Lee and Y. Pang. Phys. Rev. D 35 (1987) 3678.

[28] W. Thirring, Phys. Lett. B 127 (1983) 27.

[29] J.R. Oppenheimer and G.G. Volkoff, Phys. Rev. 55 (1939) 374.

[30] T.D. Lee, in: Multiparticle Dynamics (Festschrift for L. van Hove), eds A. Giovanni and W. Kittel (World Scientific, Singapore, 1990) p. 743.

[31] T.D. Lee, in: Proc. Symposium on Frontier Problems in High Energy Physics (in Honour of G. Bernardini). eds L. Foa and L.A. Radicati, Scuola Normale Superiore, Pisa (1976) p. 47.

[32] T.D. Lee, Particle Physics and Intoduction to Field Theory (Harwood Academic, New York, 1981).

[33] S. Coleman, in: New Phenomena in Subnuclear Physics, Part A, ed. A. Zichichi (Plenum Press, New York, 1977) p. 297.

[34] L.D. Faddeev, Lett. Math. Phys. 1 (1976) 289.

[35] J. Werle, Phys. Lett. B 71 (1977) 367.

[36] T.F. Morris, Phys. Lett. B 76 (1978) 337; 78 (1978) 87.

[37] J. Distler, B. Hill and D. Spector, Phys. Lett. B 182 (1986) 71.

[38] A.P. Balachandran, B. Rai, G. Sparano and A.M. Srivastava, Phys. Rev. Lett. 59 (1987) 853.

[39] S.Yu. Khlebnikov and M.E. Shaposhnikov, Soviet J. Nucl. Phys. 45 (1987) 747.

[40] A.M. Safian, S. Coleman and M. Axenides, Nucl. Phys. B 297 (1988) 498.

[41] K. Lee, Phys. Rev. Lett. 66 (1991) 533.

[42] Avinash Khare, Phys. Lett. B 263 (1991) 227.

[43] C.H. Lee and S.Y. Yoon, Mod. Phys. Lett. A 6 (1991) 1479.

[44] R. Jackiw, K. Lee and E.J. Weinberg, Phys. Rev. D 42 (1990) 3488.

[45] J. Baacke, Z. Phys. C 47 (1990) 619.

[46] M.A. Faria-Rosa and W.A. Rodrigues, Mod. Phys. Lett. A 4 (1989) 175.

[47] M. Creutz, Phys. Rev. D 10 (1974) 1749.

[48] A. Sym, Lett. Nuovo Cim. 40 (1984) 225.

[49] D. Loo and T.F. Morris, Hadronic J. 7 (1984) 708.

[50] P. Mathieu and T.F. Morris, Phys. Lett. B 155 (1985) 156.

[51] K.R. Subbaswamy and S.E. Trullinger, Phys. Rev. D 22 (1980) 1495.

[52] K. Chou, C. Chu, Y. Dai and Y. Wu, Scientia Sinica 23 (1980) 40.

[53] V.E. Grishin, Yu.V. Katyshev, N.V. Makhaldiani, V.G. Makhankov and A.B. Shvachka, Phys. Lett. A 78 (1980) 423.

[54] R.-K. Su and Z. Hu, Phys. Lett. A 141 (1989) 420. 
[55] R.-K. Su and Z. Hu, Phys. Lett. A 147 (1990) 115.

[56] T.D. Lee and Y. Pang, Nucl. Phys. B 315 (1989) 477

[57] E. Tomboulis, Phys. Rev. D 12 (1975) 1678.

[58] M. Creutz, Phys. Rev. D 12 (1975) 3126.

[59] J.D. Breit, Nucl. Phys. B 183 (1981) 94.

[60] L.D. Faddeev and V.E. Korepin, Phys. Rep. 42 (1978) 1.

[61] R.F. Dashen, B. Hasslacher and A. Neveu, Phys. Rev. D 10 (1974) 4114, 4130; 11 (1975) 3424.

[62] J.L. Grevais and B. Sakita, Phys. Rev. D 11 (1975) 2943.

[63] J.L. Gervais, A. Jevicki and B. Sakita, Phys. Rev. D 12 (1975) 1038.

[64] R. Rajaraman and E.J. Weinberg, Phys. Rev. D 11 (1975) 2950.

[65] C. Callan and D. Gross, Nucl. Phys. B 93 (1975) 29.

[66] A. Klein and F.R. Krejs, Phys. Rev. D 12 (1975) 3112.

[67] K. Huang and D.R. Stump, Phys. Rev. D 14 (1976) 223.

[68] K. Cahill, Phys. Lett. B 53 (1974) 174

[69] M. Creutz and K.S. Soh, Phys. Rev. D 12 (1975) 443.

[70] F. London, Superfluids, Vol. II (Dover, New York, 1964).

[71] L.D. Landau and E.M. Lifshitz, Statistical Physics, Part I (Pergamon Press, Oxford, 1980).

[72] T.D. Lee and C.N. Yang, Phys. Rev. 105 (1957) 1119.

[73] T.D. Lee, K. Huang and C.N. Yang, Phys. Rev. 106 (1957) 1135.

[74] T.D. Lee, Nature 330 (1987) 460.

[75] R. Friedberg and T.D. Lee, Mod. Phys. Lett. B 2 (1988) 469.

[76] T.D. Lee and C.N. Yang, Phys. Rev. 112 (1958) 1419; 113 (1959) 1406.

[77] G. Fai, R.J. Perry and L. Wilets, Phys. Lett. B 208 (1988) 1.

[78] G. Krein, P. Tang and A.G. Williams, Phys. Lett. B 215 (1988) 145.

[79] R. Horn, R. Goldflam and L. Wilets, Comp. Phys. Comm. 42 (1986) 105.

[80] Th. Koppel and M. Harvey, Phys. Rev. D 31 (1985) 171.

[81] L.R. Dodd and M.A. Lohe, Phys. Rev. D 32 (1985) 1816.

[82] R. Saly, Comp. Phys. Comm. 30 (1983) 411.

[83] T.D. Lee, Phys. Rev. D 19 (1979) 1802

[84] P. Tang and L. Wilets, J. Math. Phys. 31 (1990) 1661.

[85] J.-L. Dethier, R. Goldflam, E.M. Henley and L. Wilets, Phys. Rev. D 27 (1983) 2193.

[86] R.E. Peierls and J. Yoccoz, Proc. R. Soc. A 70 (1957) 381

[87] J.J. Griffin and J.A. Wheeler, Phys. Rev. 108 (1957) 311.

[88] P. Ring and P. Schuck, The Nuclear Many-Body Problem (Springer, Berlin, 1980).

[89] D.L. Hill and J.A. Wheeler, Phys. Rev. 89 (1953) 1106.

[90] A. Schuh and H.J. Pirner, Phys. Lett. B 173 (1986) 19.

[91] A. Schuh, H.J. Pirner and L. Wilets, Phys. Lett. B 174 (1986) 10.

[92] L. Wilets, in: Hadrons and Heavy Ions, Lecture Notes in Physics, Vol. 231 (Springer, Berlin, 1985) p. 317.

[93] L. Wilets, M.C. Birse, E.G. Lubeck and E.M. Henley, Nucl. Phys. A 434 (1985) 129C.

[94] E.G. Lubeck, M.C. Birse, E.M. Henley and L. Wilets, Phys. Rev. D 33 (1986) 234.

[95] E.G. Lubeck, E.M. Henley and L. Wilets, Phys. Rev. D 35 (1987) 2809.

[96] T.D. Lee and D. Pines, Phys. Rev. 88 (1952) 960; 92 (1953) 883.

[97] T.D. Lee, F.E. Low and D. Pines, Phys. Rev. 90 (1953) 297.

[98] J.R. Klauder, Ann. Phys. 11 (1960) 123.

[99] R.J. Glauber, Phys. Rev. 131 (1963) 2766.

[100] S. Mandelstam, Phys. Rev. D 11 (1975) 3026.

[101] J.G. Taylor, Ann. Phys. 115 (1978) 153.

[102] M. Bickeboller, M.C. Birse, H. Marschall and L. Wilets, Phys. Rev. D 31 (1985) 2892.

[103] E. Eichten, K. Gottfried, T. Kinoshita, K.D. Lane and T.M. Yan, Phys. Rev. D 21 (1980) 203.

[104] W. Buchmuller, Phys. Lett. B 112 (1982) 479.

[105] S.R. Gupta, S.F. Radford and W.W. Repko, Phys. Rev. D 26 (1982) 3305

[106] P. Moxhay and J.R. Rosner, Phys. Rev. D 28 (1983) 1132.

[107] H.B. Nielson and A. Patkos, Nucl. Phys. B 195 (1982) 137.

[108] G. Chanfry, O. Nachtmann and H.J. Pirner, Z. Phys. C 21 (1984) 277.

[109] A. Schuh and H.J. Pirner, Phys. Lett. B 173 (1986) 19.

[110] A.G. William and A.W. Thomas, Phys. Rev. C 33 (1986) 1070.

[111] L. Bayer, H. Forkel and W. Wiese, Z. Phys. A 324 (1986) 365

[112] H.J. Pirner, J. Wroldsen and M. Ilgenfritz, Nucl. Phys. B 294 (1987) 905. 
[113] L.R. Dodd, A.G. William and A.W. Thomas, Phys. Rev. D 35 (1987) 1040.

[114] S. Kahana, G. Ripka and V. Soni, Nucl. Phys. A 415 (1984) 351.

[115] S. Kahana and G. Ripka, Nucl. Phys. A 429 (1984) 462.

[116] M.C. Birse and M.K. Banerjee, Phys. Rev. D 31 (1985) 118.

[117] L.S. Celenza and C.M. Shakin, Phys. Rev. D 35 (1987) 2843.

[118] T.H.R. Skyrme, Proc. R. Soc. A 260 (1961) 127.

[119] E. Witten, Nucl. Phys. B 223 (1983) 422, 433.

[120] S. Chandrasekhar, An Introduction to the Study of Stellar Structure (University of Chicago Press, Chicago, 1939).

[121] G.W. Gibbons and S.W. Hawking, Phys. Rev. D 15 (1977) 2753.

[122] R. Arnowitt, S. Deser and C.W. Misner, in: Gravitation: an Introduction to Current Research, ed. L. Witten (Wiley-Interscience, New York 1962) p. 227.

[123] M. Colpi, S.L. Shapiro and I. Wasserman, Phys. Rev. Lett. 57 (1986) 2485.

[124] J.J. van der Bij and M. Gleiser, Phys. Lett. B 194 (1987) 482

[125] M. Gleiser, Phys. Rev. D 38 (1988) 2376.

[126] P. Jetzer, Phys. Lett. B 231 (1989) 433.

[127] N.K. Glendenning and B. Banerjee, Phys. Rev. C 34 (1986) 1072.

[128] B.W. Lynn, Nucl. Phys. B 321 (1989) 465.

[129] S. Bahcall, B.W. Lynn and S.B. Selipsky, Nucl. Phys. B 325 (1989) 606.

[130] S. Bahcall, B.W. Lynn and S.B. Selipsky, Nucl. Phys. B 331 (1990) 67.

[131] K. Griest, E.W. Kolb and A. Massarotti, Phys. Rev. D 40 (1989) 3529.

[132] K. Griest and E.W. Kolb, Phys. Rev. D 40 (1989) 3231.

[133] J.A. Frieman, A.V. Olinto, M. Gleiser and C. Alcock, Phys. Rev. D 40 (1989) 3241.

[134] K.-W. Ng and W.K. Sze, Phys. Rev. D 43 (1991) 3813. 